\title{
Pathophysiology and clinical implications of lower respiratory tract infection (LRTI) with respiratory enteric orphan virus (reovirus): Background and experimental evidence
}

\author{
Maret E. Bernard \\ West Virginia University
}

Follow this and additional works at: https://researchrepository.wvu.edu/etd

\footnotetext{
Recommended Citation

Bernard, Maret E., "Pathophysiology and clinical implications of lower respiratory tract infection (LRTI) with respiratory enteric orphan virus (reovirus): Background and experimental evidence" (2010). Graduate Theses, Dissertations, and Problem Reports. 3227.

https://researchrepository.wvu.edu/etd/3227
}

This Thesis is protected by copyright and/or related rights. It has been brought to you by the The Research Repository @ WVU with permission from the rights-holder(s). You are free to use this Thesis in any way that is permitted by the copyright and related rights legislation that applies to your use. For other uses you must obtain permission from the rights-holder(s) directly, unless additional rights are indicated by a Creative Commons license in the record and/ or on the work itself. This Thesis has been accepted for inclusion in WVU Graduate Theses, Dissertations, and Problem Reports collection by an authorized administrator of The Research Repository @ WVU. For more information, please contact researchrepository@mail.wvu.edu. 
PATHOPHYSIOLOGY AND CLINICAL IMPLICATIONS OF LOWER RESPIRATORY TRACT INFECTION (LRTI) WITH RESPIRATORY ENTERIC ORPHAN VIRUS (REOVIRUS):

BACKGROUND AND EXPERIMENTAL EVIDENCE

by

Maret E. Bernard

Thesis submitted to the School of Medicine

at West Virginia University

in partial fulfillment of the requirements

for the degree of

\author{
Master of Science \\ in \\ Biomedical Science
}

Approved by

Mario Scuri, M.D., Ph.D., Committee Chairperson

Rosana Schafer, Ph.D.

Christopher Cuff, Ph.D.

Department of Microbiology, Immunology \& Cell Biology

Morgantown, West Virginia

2010

Keywords: Reovirus, Neurogenic Inflammation, Caveolin-1, Prostaglandin, Leukotriene, Fibrosis and Airway Remodeling 


\section{ABSTRACT \\ PATHOPHYSIOLOGY AND CLINICAL IMPLICATION OF LOWER RESPIRATORY TRACT INFECTION (LRTI) WITH RESPIRATORY ENTERIC ORPHAN VIRUS (REOVIRUS): BACKGROUND AND EXPERIMENTAL EVIDENCE}

\section{BY MARET E. BERNARD}

Background. Respiratory viral infection early in life is a predominant factor in the inception of episodic wheezing and development of asthma amongst young children [1] and a serious health challenge. Previous studies of lower respiratory tract infections (LRTI) with respiratory syncytial virus (RSV) in animal models have indicated that early life viral exposure results in dysregulated neuroimmune interactions and altered synthesis/release of proinflammatory neuropeptides generating increased airway reactivity and neurogenicinflammation. Similar to RSV, respiratory enteric orphan virus (Reovirus) is a common respiratory pathogen associated with pulmonary infections in children. Also, reovirus pulmonary infection has been shown to induce increased collagen deposition and be associated with the pathogenesis of bronchiolitis obliterans with organizing pneumonia (BOOP). In this study, we investigated the effects of reovirus exposure on physiological airway responses and whether these responses were associated with neurogenic inflammation and airway remodeling.

Methods. Adult (12 weeks) and weanling (2 weeks) Fisher-344 (F-344) rats were infected with reovirus or a pathogen-free vehicle and the changes in airway vascular permeability, neurotrophin expression, inflammatory response and protein content were measured at either 5 or 30 days after infection to determine changes in neurogenic inflammation and airway remodeling.

Results. Neurogenic inflammation increased in all treated animals 5 days after inoculation and up to 30 days in adult rats. This effect was not associated with any changes in nerve growth factor (NGF) and brain-derived neurotrphic factor (BDNF) expression in any animals at both time points. All treated animals developed acute pneumonia which resolved at 30 days. However, weanling rats showed mild peri-alveolar fibrosis at 30 days.

Conclusions. Reovirus potentiates neurogenic inflammation in rat airways. This effect is not associated with changes in neurotrophin expression. In weanling rats, reovirus infection induced peri-alveolar fibrosis suggesting that early exposure may carry long-term effects which may be clinically relevant. 


\section{Acknowledgement}

First and foremost, I would like to acknowledge my advisor and committee chair, Dr. Mario Scuri, whose patience, supervision, advice and guidance was central in completing this work. I would also like to acknowledge the members of my committee, Dr. Cuff and Dr. Schaefer, whose advice, perceptive incite and guidance were significant components in this process. I greatly appreciated the effort by Dr. Rosanna Schaefer whose supervision, concerned guidance and counsel, gave me the necessary means to complete this program. She acted as my advocate, taking the extra time to assist and help me and for that I am grateful. I am also indebted to Dr. Christopher Cuff, whose critical advice and informed contributions were essential in directing my studies.

I owe a debt of graditude to the Department of Pediatrics, specifically Dr. Giovanni Piedimonte, for his advice, ideas and crucial contributions that helped direct this research. Similarly, I would like to thank the members of the Pediatric Research lab, Debbie Piktel and Cheryl Walton, whose tutelage was very helpful. I am also grateful to another member of the Pediatric lab, Lennie Samsell, for taking the time to teach and encourage me in the lab. Her guidance and assistance made these results possible.

During my graduate experience, I had the pleasure of meeting and forming tremendous friendships with different colleagues, specifically Amy Jones, Amanda Buskirk, Lance Wollenberger and Mathew Novotany. I am fortunate to have met these individuals and grateful for their help and support to finish this program. I also want to thank Fritz Schaupp, for being my devoted companion that supported and encouraged me during this process.

I want to express my appreciation to Dr. Jeffrey Cohen who has been a mentor to me and whose guidance and advice has been indispensable in helping me find my way. 
I owe my deepest and sincerest gratitude to my mom and dad, Suzanne and George Bernard, for being my devoted and uncompromising advocates. The selfless sacrifices they have made for me and the infinite support, love, guidance, and understanding they have bestowed upon me have allowed me to achieve my goals and to become who I am. It is an honor to be their daughter and I am grateful to them. 


\section{Table of Contents}

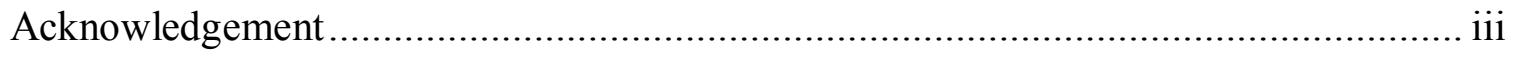

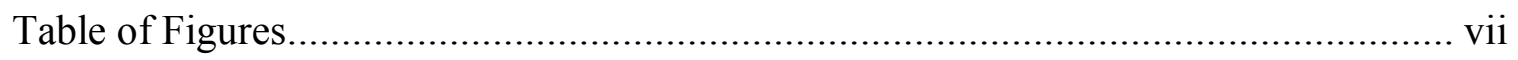

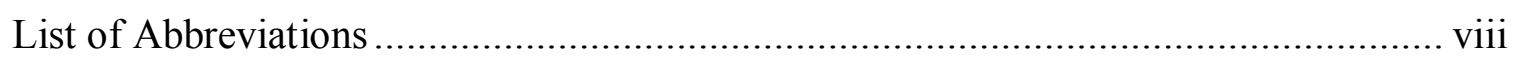

Respiratory Enteric Orphan Virus .......................................................................

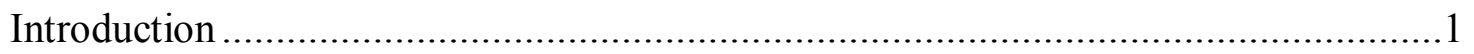

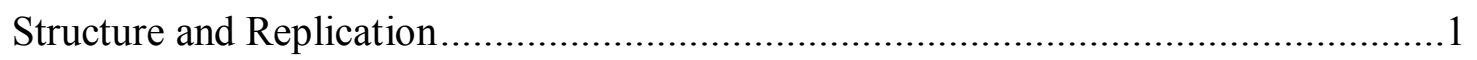

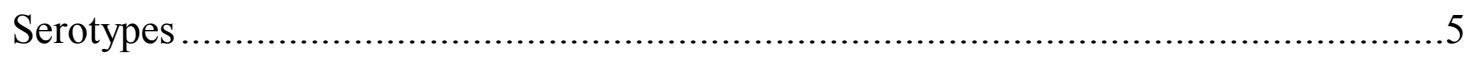

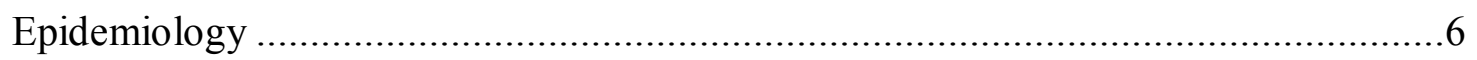

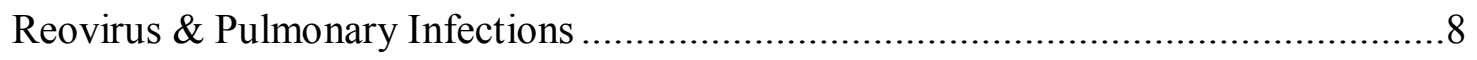

Reovirus and Bronchiolitis Obliterans Organizing Pneumonia (BOOP) .....................9

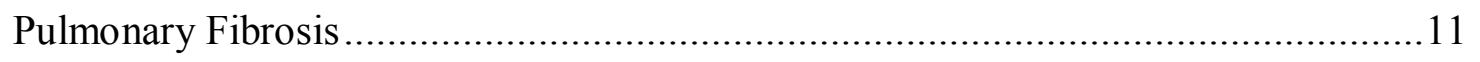

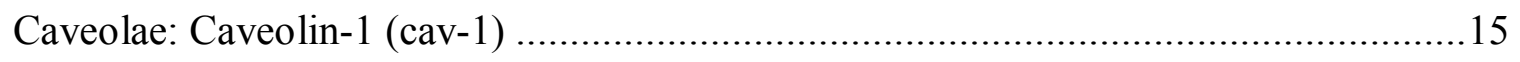

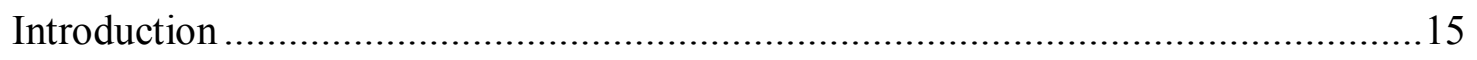

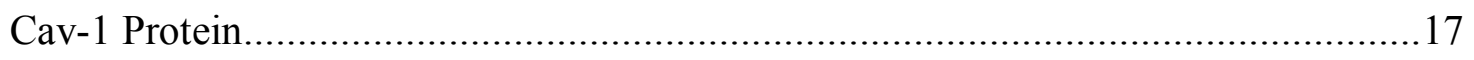

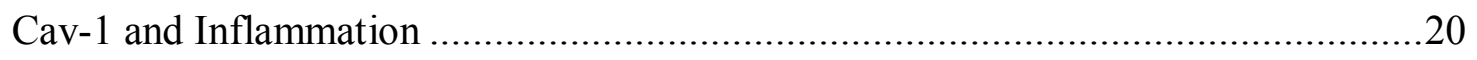

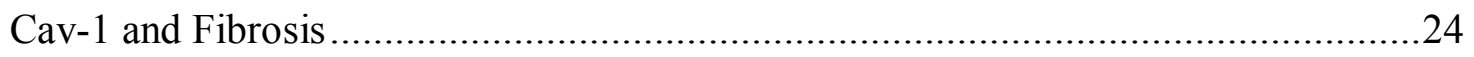

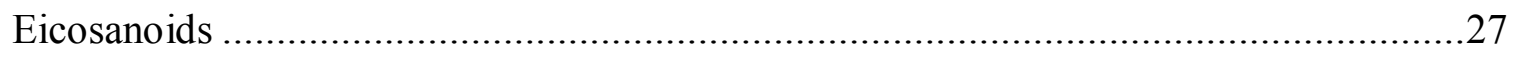

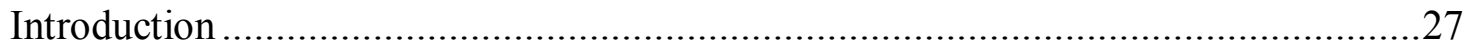

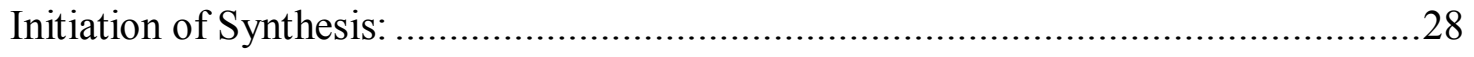

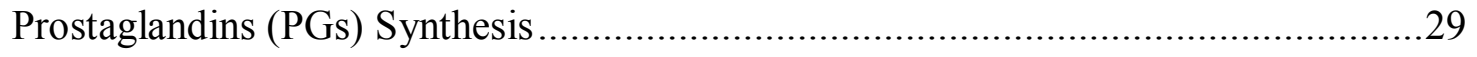

Mechanism of PG Signaling: Receptors and Cellular Effects .......................................31

The Role of PGs in Lung Inflammation .................................................................

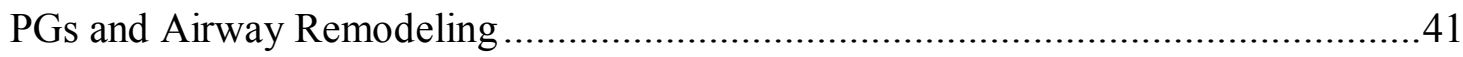

Leukotrienes (LTs) Synthesis.........................................................................45

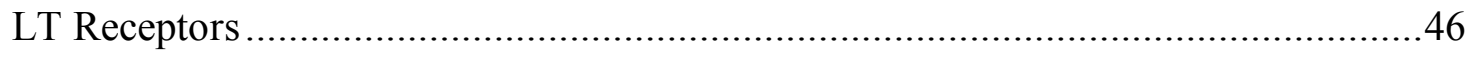

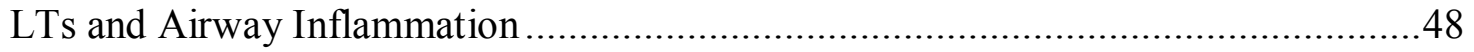

Leukotrienes and Airway Remodeling ..............................................................54

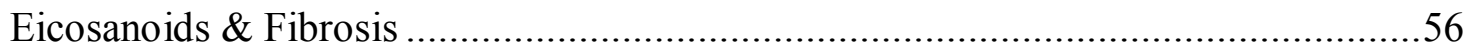

Effects of Reovirus Exposure on Airway Reponses in an Experimental Model of Lower

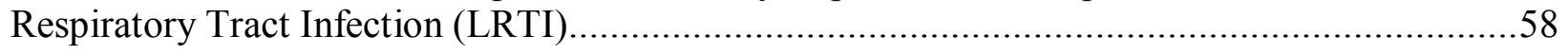

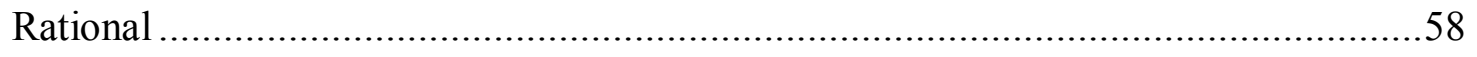

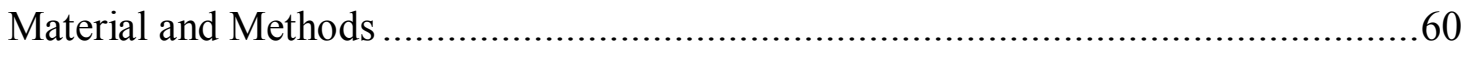

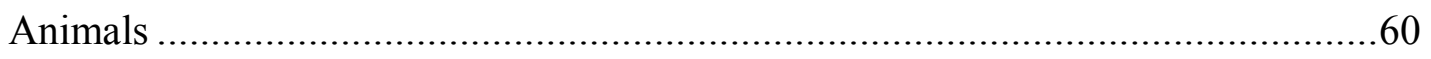




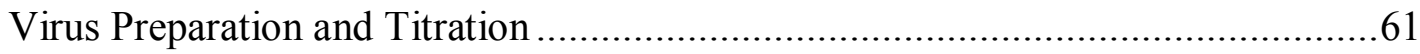

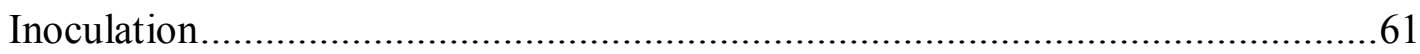

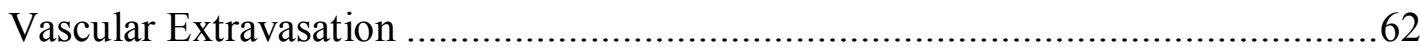

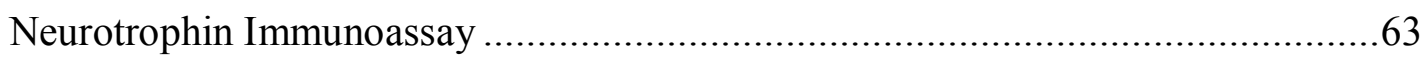

Reverse Transcription-Polymerase Chain Reaction (RT-PCR) ………………........63

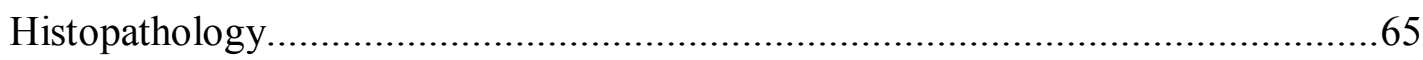

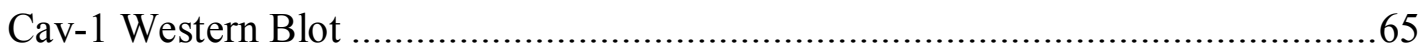

Quantification of $\mathrm{PGE}_{2}$ by enzyme immunoassay (EIA)......................................66

Quantification of CysLT by enzyme immunoassay (EIA) ………………….........67

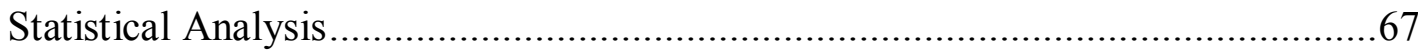

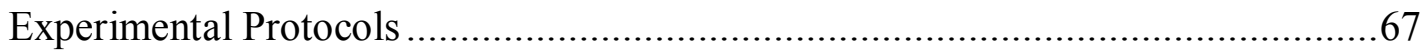

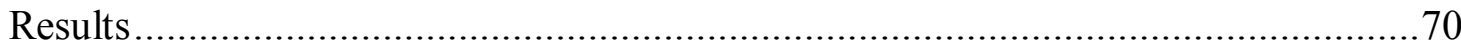

HiSTOPATHOLOGY AND MORPHOMETRIC ANALYSIS .......................................

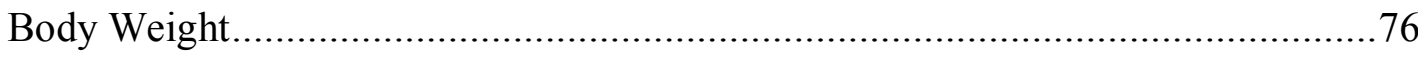

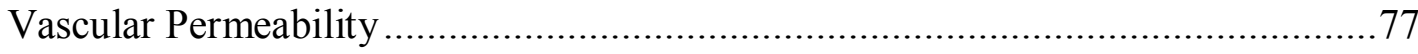

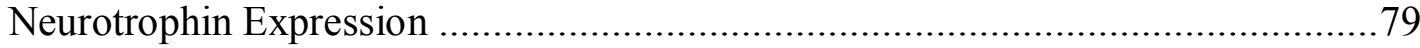

Caveolin-1 Protein Expression ............................................................................. 81

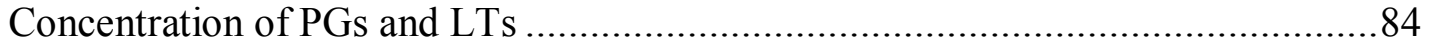

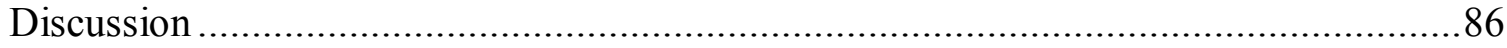

Maret Elizabeth Bernard ……………..........................................................112 


\section{Table of Figures}

Figure 1 Structure and Maturation of Reovirus ...........................................................2

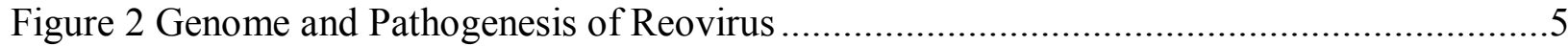

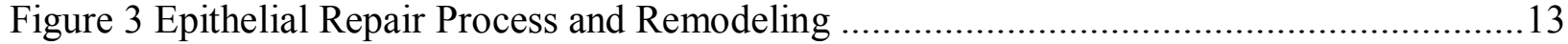

Figure 4 Altered Repair Results in Fibrosis ............................................................... 14

Figure 5 Membrane Topology, Domains and Signaling .................................................... 16

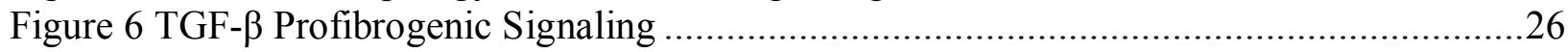

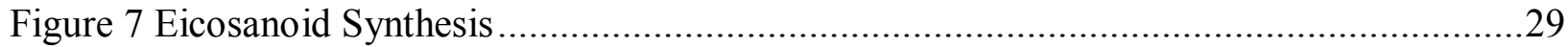

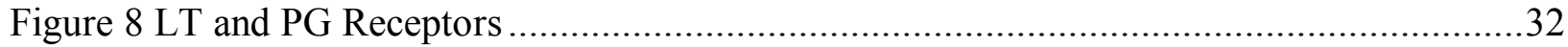

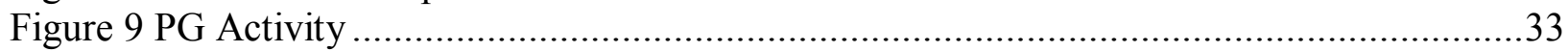

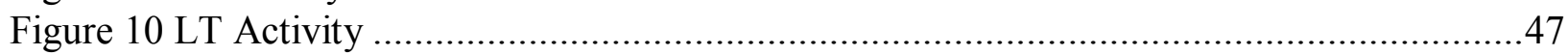

Figure 11 Histological features of reovirus induced pneumonia at 5 and 30 days post infection.71

Figure 12 Histological features of reovirus induced pneumonia in adult and weanling rats ........72

Figure 13 Trichrome Staining and Morphometric Analysis............................................. 74

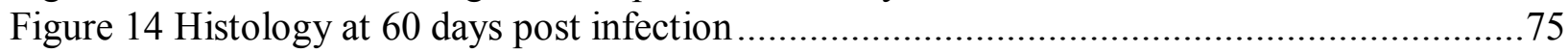

Figure 15 Adult Rat Bronchus-associated Lymphoid Tissue (BALT) .................................76

Figure 16 Acute Changes in Body Weight Demonstrate Reovirus Pathology..........................77

Figure 17 Changes in Vascular Permeability indicative of Neurogenic Inflammation ...............78

Figure 18 Neurotrophin Protein Expression at $5 \& 30$ days ............................................. 80

Figure 19 mRNA Expression of Neurotrophins and their Receptors ...................................81

Figure 20 Analysis of Cav-1 Protein Expression post reovirus infection ...............................83

Figure 21 Expression of the hormones PGE2 and LT in response to reovirus infection.............85 


\section{List of Abbreviations}

5-LO: 5-Lipoxygenase

$\alpha$-SMA: $\alpha$ - Smooth Muscle Actin

AEC: Alveolar Epithelial Cell

AHR: Airway Hyper Responsiveness

APC: Antigen Presenting Cell

AM: Alveolar Macrophages

AMDGF: Alveolar Macrophage Derived Growth Factor

AR: Androgen Receptor

ASM: Airway Smooth Muscle

BAL: Bronchoalveolar Lavage

BDNF: Brain-Derived Neurotrophic Factor

BLT: B Leukotriene Receptor

BOOP: Bronchiolitis Obliterans Organizing Pneumonia

cAMP: Cyclic Adenosine Monophosphate

Cav-1: Caveolin-1

Cox-1or-2: Cyclooxygenase-1 or -2

CSD: Caveolin Scaffolding Domain

Cys: Cysteine

cysLT: Cysteinyl Luekotrienes

cysLT $_{1}$ or cysLT $T_{2}$ : Cysteinyl Leukotriene Receptor 1 or 2

DC: Dendritic Cell

Der-f: Dermatophagoides farinea 
DP: D Prostanoid Receptor

dsRNA: Double Stranded Ribonucleic Acid

ECM: Extracellular Matrix

EGF- Epidermal Growth Factor

EGFR: Epidermal Growth Factor Receptor

ELISA: Enzyme-Linked Immunosorbent Assay

EM: Electron Microscopy

eNOS: Endothelial Nitric Oxide Synthase

ER: Endoplasmic Reticulum

ERK: Extracellular Signal-Regulated Kinase

EstR: Estrogen Receptor

ET-1: Endothelin-1

FceRI: Fc Receptor for IgE

FGF: Fibroblast Growth Factor

FLAP: 5-Lipoxygenase Activating Protein

fMLP: Formyl-Met-Leu-Phe

GM-CSF: Granulocyte Macrophage-Colony Stimulating Factor

GPCR: G-Protein Coupled Receptor

HA: Hemaglutination

HBoV: Human Boca Virus

H-Ras: v-Ha-Ras Harvey Rat Sarcoma Viral Oncogene Homolog hMPV: Human Metapneumovirus

ICER: Inducible cAMP Early Repressor 
Ig: Immunoglobulin

IGF: Insulin Growth Factor

IL: Interleukin

$\mathrm{IP}_{3}$ : Inositol Trisphosphate

IPF: Idiopathic Pulmonary Fibrosis

ISVP: Infectious Sub-Viral Particle

JAM: Junction Adhesion Molecule

JNK: Jun-N-Terminal Kinase

KC: Keratinocyte-Derived Cytokine

LN: Lymph Node

LPS: Lipopolysaccharide

LRTI: Lower Respiratory Tract Infection

LT: Leukotrienes

LTA $_{4}:$ Leukotriene $\mathrm{A}_{4}$

$\mathrm{LTB}_{4}$ : Leukotriene $\mathrm{B}_{4}$

$\mathrm{LTC}_{4}$ : Leukotriene $\mathrm{C}_{4}$

LTD $_{4}$ : Leukotriene $\mathrm{D}_{4}$

LTE4: Leukotriene $\mathrm{E}_{4}$

MAPK: Mitogen Activated Protein Kinase

MC: Mast Cell

MCP-1: Monocyte Chemotractant Protein-1

MIP-2: Macrophage Inflammatory Protein-2

MoDC: Monocyte Derived Dendritic Cell 
mPGES-1 or -2: Microsomal PGE Synthase -1 or -2

mRNA: Messenger Ribonucleic Acid

NEP: Neutral Endopeptidase

NF-кB: Nuclear Factor Kappa-Light-Chain-Enhancer of Activated B Cells

NGF: Nerve Growth Factor

NK-1: Neurokinin-1

NO: Nitric Oxide

OVA: Ovalbumin

PAF: Platelet Activating Factor

PDGFR: Platelet Derived Growth Factor Receptor

PF: Pulmonary Fibrosis

PG: Prostaglandin

$\mathrm{PGE}_{2}$ : Prostaglandin $\mathrm{E}_{2}$

$\mathrm{PGD}_{2}$ : Prostaglandin $\mathrm{D}_{2}$

PGHS-1 or -2: Prostaglandin Endoperoxide H Synthase-1 or -2

PGT: Prostaglandin Transporter

PI3K: Phosphatidylinositol 3-Kinase

PIV: Parainfluenza Virus

PKA: Protein Kinase A

PKC: Protein Kinase C

PM: Peritoneal Macrophages

PMN: Polymorphonuclear Cell or Neutrophil

PS: Phosphatidylserine 
PTEN: Phosphatase and Tensin Homolog

Raf-1: V-Raf-1 Murine Leukemia Viral Oncogene Homolog 1

Reovirus: Respiratory Enteric Orphan Virus

RNA: Ribonucleic Acid

RSV: Respiratory Syncytial Virus

siRNA: Small Interfering RNA

SP: Substance P

T1L: Type 1 Lang

T3D: Type 3 Dearing

TCR: T Cell Receptor

TGF: Transforming Growth Factor

TGF- $\beta$ R: TGF- $\beta$ Receptor

Tg-MPM: Thioglycollate-Elicited Mouse Peritoneal Macrophage

$\mathrm{T}_{\mathrm{h}} 1: \mathrm{T}$ Helper 1 Cell or Type $1 \mathrm{~T}$ Helper Cell

$\mathrm{T}_{\mathrm{h}} 2$ : $\mathrm{T}$ Helper 2 Cell or Type $2 \mathrm{~T}$ Helper Cell

$\mathrm{T}_{\mathrm{h}}$ 17: T Helper 17 Cell

TMD: Transmembrane Domain

TNF: Tumor Necrosis Factor

$\mathrm{TXA}_{2}$ : Thromboxane 


\section{CHAPTER 1}

\section{Respiratory Enteric Orphan Virus}

\section{Introduction}

Respiratory enteric orphan viruses (Reoviruses) [2] are commonly found in the airways of exposed infants early in life [3-5] and are associated with pulmonary infections and the common cold [6]. Reovirus has been extensively studied as an ideal experimental model of viral pneumonia [7], however little is known about the pathologic mechanisms of reovirus-induced pulmonary infection.

Reoviruses were first isolated from healthy children's stool in 1954 and classified as a member of the ECHO type 10 viruses. It was later determined that the virus had distinctive characteristics from other members of the ECHO type 10 group and was officially identified by Sabin as a respiratory enteric orphan on the basis of its repeated isolation from respiratory and enteric tracts of asymptomatic and symptomatic children [5]. Common symptoms associated with reovirus infection are mild, cold-like respiratory difficulties such as a cough or gastrointestinal problems, specifically diarrhea. Current evidence of other clinical manifestations are incomplete and patchy, but have demonstrated that reovirus has a wide range of infectivity, but predominantly infects the respiratory and gastrointestinal tract [8]. Long term effects of clinical sequelae following viral infection have not been fully elucidated.

\section{Structure and Replication}

Mammalian reoviruses are nonenveloped, cytoplasmically replicating viruses. The viral structure consists of an outer casing and an internal core. The outer casing is comprised of two concentric protein capsids interspersed with fibrous protein spike extensions that generate an 
appearance described as icosahedral geometry (Figure 1-C). The outer capsid surrounds the viral core that contains the virus's genome. The genome consists of 10 segments of double stranded ribonucleic acid (dsRNA) that are grouped into three categories corresponding to their size: $\mathrm{L}$ (large), M (medium) and S (small) (Figure 1-A). Segments range from $3.9 \mathrm{kbp}$ to $1 \mathrm{kbp}$ and each segment encodes 1-3 proteins with the S1 gene segment being the only bicistronic gene [9] (Figure 2-A). The $\mathrm{S} 1$ gene encodes the viral attachment proteins $\sigma 1, \sigma 2$ and $\sigma 3$ that form the fibrous spikes that extend from the $\mu 1$ protein outer capsid which is encoded by the M2 gene [10]. The inner capsid is made up of the $\mu 2$ protein encoded on the M2 gene and is the site of origin for the viral spikes [11] (Figure 1-C). The viral core consists of the proteins $\lambda 1, \lambda 2$ and $\lambda 3$ that are responsible for producing the necessary tools for viral replication [12].
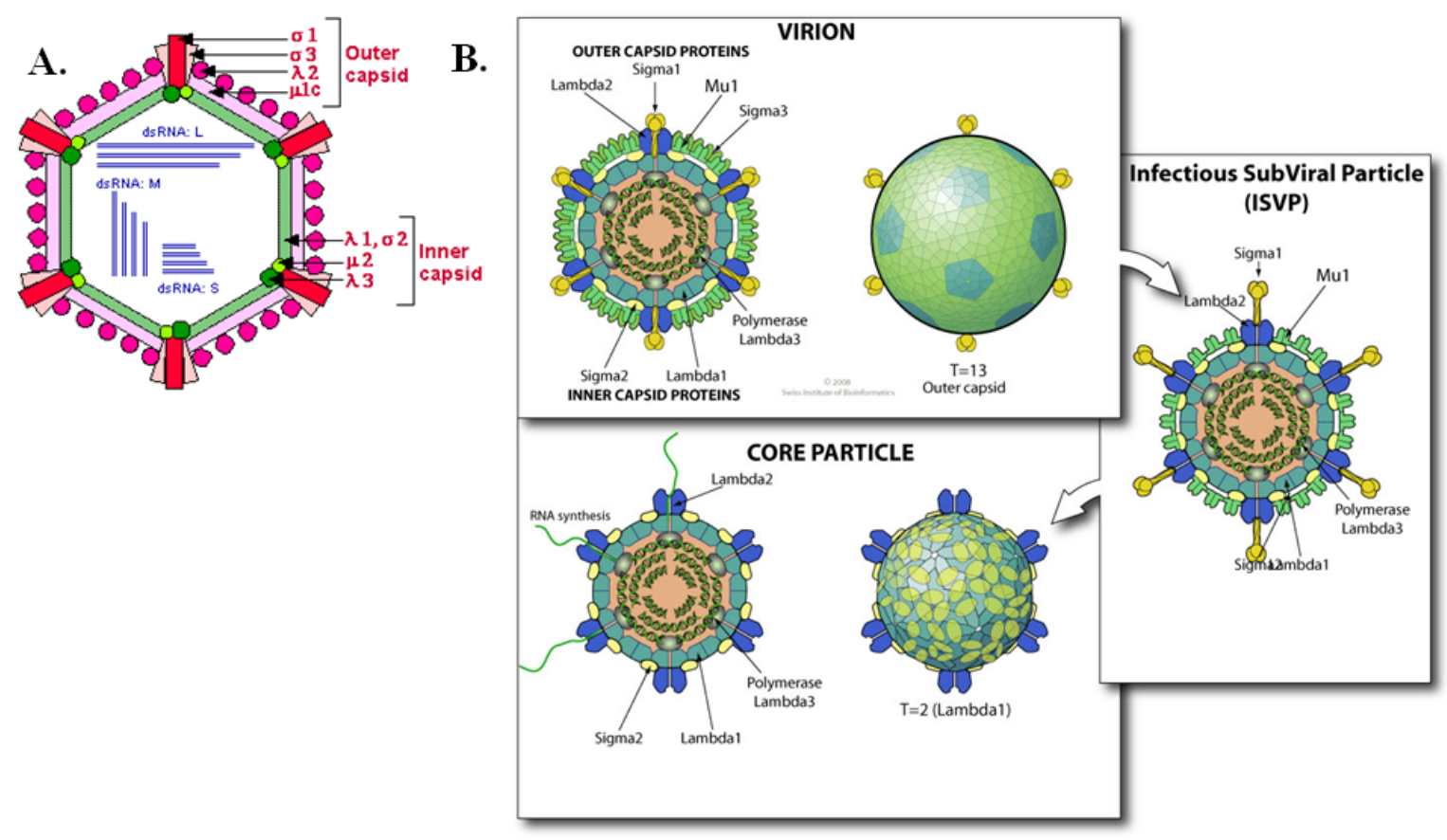

\section{Figure 1 Structure and Maturation of Reovirus}

A) Is a schematic representation of reovirus structure showing the three main components: inner capsid, outer capsid and dsRNA genome. (adapted from ViralZone reference [13]). B) An illustration of the different stages of reovirus disassembly starting with the nonenveloped, icosahedaral virion $(80 \mathrm{~nm})$ composed of an outer protein capsid $(\sigma 3$ and $\mu 1)$, an inner protein capsid $(\sigma 1)$ and the attachment proteins ( $\sigma 1$ and $\lambda 2$ ). The outer capsid has an icosahedral $\mathrm{T}=13$ symmetry and the inner capsid has an icosahedral $\mathrm{T}=2$. Removal of $\sigma 3 / \mathrm{VP} 7$ results in the second viral stage known as infectious subviral particles (ISVPs). ISVPs are then further disassembled to form the core particle $(\lambda 1, \lambda 2$ and $\lambda 3)$ that produces the tools for viral replication.(adapted from ViralZone reference [13]). 
Reovirus infection is a step wise process where the virion first attaches to the host cell via an interaction between the $\sigma 1$ viral protein and the host cell's sialic acid and junction adhesion molecules (JAM) (Figure 2-B). Following attachment, the virus undergoes endocytosis and is transported in endosomes. Once inside the endosome virions undergo partial cleavage or are progressively uncoated through an acidic $\mathrm{pH}$ and cysteine-protease dependent process. This results in virions being converted into infectious sub-viral particles (ISVP) (Figure 1-B \& C). Conversion to ISVPs requires the removal of the major capsid protein $\sigma 3$, cleavage of the major outer capsid protein $\mu 1$ into smaller virion-associated fragments and conformational changes to the attachment protein $\sigma 1$. Conversion to ISVPs can also occur extracellularly through the action of proteolytic enzymes, such as those found in the intestines, and act as the primary infectious particle. In this situation the ISVPs infect cells via endocytosis, but they no longer require endosomal acidification for processing (Figure 2-B). Regardless of their site of initial generation, ISVPs are processed further within endosomes to form the non-infectious but transcriptionally active core particle. This transformation requires the removal of the $\sigma 1$ and virion-associated $\mu 1$ fragments and is associated with the process of penetration through the endosomal membrane by the core particle. The decisive event in the process is the exposure of $\mu 1$ residues that interact and initiate fusion of the ISVP with the endosomal membrane, providing a method of delivery of core particles into the cytoplasm. At this point conservative transcription of the genome occurs. The virion RNA is copied by the viral RNA polymerase while still in the core. The dsRNA genome does not function as a messenger RNA (mRNA), making the production of mRNA the first initial step of the process. The mRNA is copied from the negative strand template found in the dsRNA within the parental cores during primary transcription. This results in production of numerous capped transcripts of positive strand RNA that are not 
polyadenylated. Capped mRNAs leave the core and enter into cytoplasm through channels in the $\lambda 2$ core spike. The extruded capped mRNAs have two different fates. Some of the mRNAs are translated to form viral proteins and are self-assembled to form the viral capsid and cores through a process still not completely understood. mRNAs that are not transcribed are packaged into virions where they act as a template for the negative strand to form a complete dsRNA. Progeny virus particles assemble in the cytoplasm 6-7h after infection, forming inclusion bodies that can be seen in the infected cell. Nascent virus particles are released when the infected cell undergoes apoptosis and/or lysis [14]. 
A.

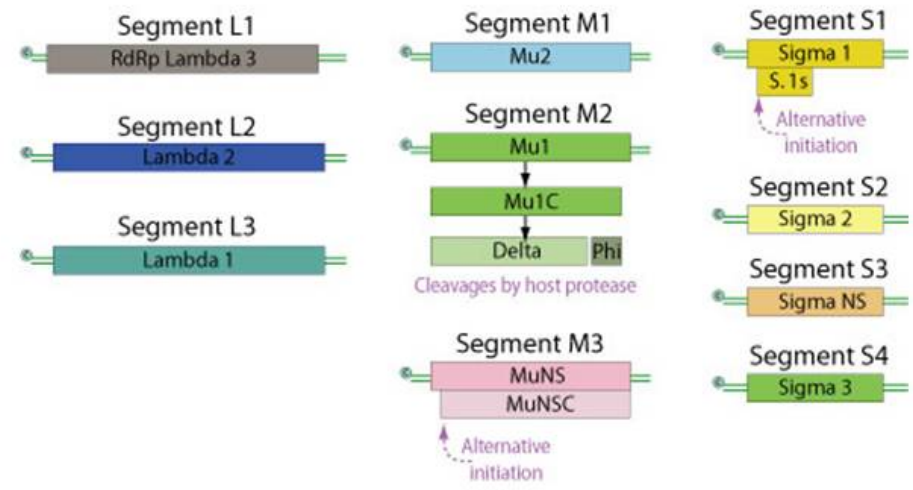

B.

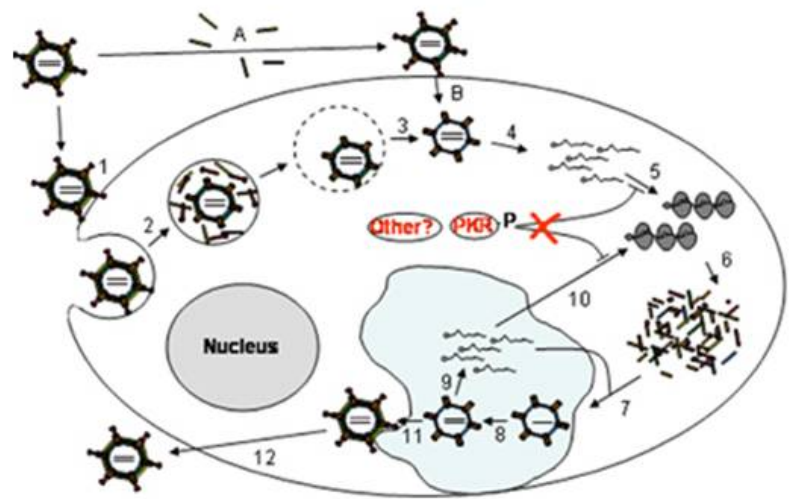

Reprinted by perm ission from Macm illan Publishers Ltd: Oncogene [4], Copyright (1) 2005, Nature Publishing Group (2010) http//dx.doi.org/10.1038/si.onc.1209041

\section{Figure 2 Genome and Pathogenesis of Reovirus}

A) Reovirus has a segmented dsRNA linear genome that contains 10 segments: L1, L2, L3, M1, M2, M3, S1, S2, S3, S4, coding for 12-13 proteins. Segment size is 1,189-3,916 nt and total genome size is $23.5 \mathrm{~Kb}$. (Adapted from ViralZone reference [13]). B) Reovirus life cycle: (1) attachment via the interactions between the viral s1 protein and host cell sialic acid and/or JAM receptors; (2) receptor-mediated endocytosis induces shedding of $\sigma 1$ and $\sigma 3$ proteins; (3) $\mu 1$ is cleaved by endocytic proteases and exits the endosome; (4) primary transcription occurs within reovirus cores and capped mRNAs are released; (5) primary translation of all $10 \mathrm{mRNAs}$ by host ribosomes; (6) viral proteins accumulate in viral factories produced by viral nonstructural proteins; (7) new cores assemble; (8) synthesis of minus-strand of viral RNA; (9) secondary transcription occurs within new viral cores; (10) secondary translation amplifies viral proteins; (11) complete assembly of outer capsid; (12) release follows cells lysis or apoptosis. An alternate route is the endocytosis of ISVPs. Reprinted by permission from Macmillan Publishers Ltd: Oncogene [15], Copyright 2005, Nature Publishing Group (2010) http://dx.doi.org/10.1038/sj.onc.1209041

\section{Serotypes}

Currently there are three distinct reovirus serotypes that can be differentiated by testing hemagglutination (HA) doses [16] and clinical symptoms. The original prototype of reovirus is the type 1 Lang strain (T1L) that was recovered from healthy children or those suffering from mild febrile illness [5]. Type 2 reovirus, referred to as the Jones strain, was isolated from children suffering from diarrhea, steatorrheic enteritis or that were healthy subjects [8]. The 
virus was also isolated from chimpanzees with rhinitis and monkeys suffering from pneumonia. Type 3 Dearing strain (T3D) was recovered from children suffering from diarrhea or upper respiratory infections [8]. Combined, all three reoviruses demonstrate specific cytopathogenic effects that can directly infect all mammals, including humans, and can be found in any area of the world. The vast dispersal and general ease of viral infection make reovirus an important and common virus.

\section{Epidemiology}

Investigations of the prevalence of reovirus exposure and/or infection have revealed a high incidence level of viral contact amongst children as well as adults. The seroprevalence of antibodies against reovirus has revealed a high occurrence of seropositivity demonstrating a considerable frequency of reovirus exposure. Further evidence of the frequency of reovirus exposure is demonstrated by the fact that $50 \%$ of adult sera and $25 \%$ of childhood sera from patients with respiratory infection are positive for reovirus antibodies [17-18]. In 1962, the earliest study of reovirus seroprevalence was conducted by Lerner et al. [19] where 235 sera samples were collected randomly from patients whose ages ranged from premature infants to 60 year old adults. Results from mothers and their newborn infants demonstrated that antibodies to all reovirus serotypes pass freely through the placenta and provided passive immunity to infants up until 6 months of age, after which anti-reovirus antibodies dissipate. Anti-reovirus antibodies did not begin to appear again until early childhood with $50 \%$ of sera being positive by the age of 10 years old. The highest amount of sero-positive sera was found in the age group of 41-60 years old with more than $80 \%$ of the patients testing positive for antibodies to reovirus. This demonstrated that the increase of anti-reovirus antibodies in children after loss of passive immunity was gained through reovirus exposure early in childhood. Collectively, this indicates 
that reovirus is a common infective agent affecting children. Additionally, a study done by Leers and Rozee [17] looked at the incidence of antibodies to all three reovirus serotypes in children ages 1 month to 10 years in an urban setting. They determined that serotypes 2 and 3 demonstrated the decline of maternal antibodies described by Lerner et al., but found something completely different with the serotype T1L. They determined that the "pattern for T1L antibodies demonstrated a process of active infantile infection during the time of collection." T1L antibodies showed a gradual increase during the first year of life that was then followed by a more rapid increase during the early school years, which then plateaus by the age of 10 . Once again, this demonstrates that active reovirus infections occur very commonly amongst young children.

In a more recent study, Tai et al [20] collected 273 serum specimens from healthy fullterm infants that were followed until 5 years of age. Serum was tested for anti-reovirus Immunoglobulin (Ig), specifically $\operatorname{IgA}, \operatorname{IgG}$ and $\operatorname{IgM}$ antibodies via indirect enzyme-linked immunosorbent assay (ELISA). They found that $75 \%$ of infants $0-3$ months of age were positive for anti-reovirus antibodies which decreased to $11.1 \%$ in 3-6 month old infants and totally disappeared to $0 \%$ in children 6-12 months old. Seroprevalence began to rise again with $8.2 \%$ of children 1-2 years of age being positive for anti-reovirus antibodies. This percentage increased to $32.8 \%$ in children ages $4-5$ years old and grew to $50 \%$ in children ages $5-6$ years of age. These findings mimicked those of the earlier studies and demonstrated that reovirus infections are common during early childhood following the loss of passive immunity. The high rate of reovirus infection among young children, adults and elderly, consistently indicated by epidemiological evidence, makes this type of infection even more important regarding its 
potential relevant impact on the public. However, the mechanisms behind reovirus pathogenesis and their long term effects on airway responses are still not fully elucidated.

\section{Reovirus \& Pulmonary Infections}

Reovirus pulmonary infection has been documented by numerous studies that have clarified the entry, pathogenesis and clearance of reovirus from pulmonary tissue and lymph nodes (LN) $[6,21]$. Reovirus enters the respiratory tract through pulmonary $\mathrm{M}$ cells and spreads through the airway and into regional lymph nodes resulting in viral pneumonia [22]. Studies by Morin et al. characterized reovirus infection as an ideal model of viral pneumonia [7]. They demonstrated that intratracheal inoculation with T1L resulted in a pneumonia characterized by an influx of inflammatory cells into the alveolar spaces, resulting in epithelial damage as indicated by type II alveolar epithelial cell hyperplasia [7]. The inflammatory cells in the alveolar spaces were comprised of neutrophils, mononuclear cells and lymphocytes [23]. Both CD4 and CD8 T cells, as well as B cells, make up the lymphocyte population present in both the alveoli and interstitium of the lungs [24]. It was determined by Morin and colleagues [7] that reovirus replication is necessary for the development of pulmonary inflammation and the development of pneumonia. Viral particles have been isolated from type I alveolar epithelial cells and in macrophages suggesting that within these cell types viral replication was occurring. Viral replication in alveolar type I epithelial cells is the primary factor responsible for inducing epithelial cell damage and loss resulting in type II cell hyperplasia and increased inflammation [7]. Alveolar macrophages are responsible for removing apoptotic tissue and other foreign material from alveolar spaces. Macrophage function provides an ideal means for viral entry into the cell and a key factor in reovirus pathogenesis. Reovirus replication in macrophages could alter the host response by interfering with chemokine and cytokine production and lymphocyte 
activation. For example, neutrophil chemotactic factors keratinocyte-derived cytokine (KC) and macrophage inflammatory protein-2 (MIP-2) produced by macrophages can be altered by reovirus exposure, indicating the potential for reovirus influence [25]. This effect is serotype specific according to viral protein expression and needs to be further studied. Therefore, inflammation in response to reovirus infection of the lungs is characterized by inflammatory cell influx and viral replication in alveolar cells that results in type II cell hyperplasia.

The immune response to primary infection is associated with the development of both a type 1 helper $\mathrm{T}$ cell $\left(\mathrm{Th}_{1}\right)$ and type 2 helper $\mathrm{T}$ cell $\left(\mathrm{Th}_{2}\right)$ response, resulting in the production of a humoral and cell mediated immune response. The Th1 immune response is dependent upon the activation of both CD4 and CD8 T cells [26] to produce antiviral cytokines such as interferon- $\gamma$ (IFN- $\gamma$ ) and interleukin-12 (IL-12) inducing cell mediated immunity. The expression of $\mathrm{Th}_{1}$ cytokines also affects the humoral immune response by inducing antibody class switching to IgG2a [27]. The $\mathrm{Th}_{2}$ immune is characterized by the production of IL- 6 and IL-10 that results in a humoral immune response resulting in IgG1 antibody production [27]. Therefore, viral clearance and immunity is dependent upon both humoral and cell mediated immunity.

\section{Reovirus and Bronchiolitis Obliterans Organizing Pneumonia (BOOP)}

\section{Background}

Bronchiolitic obliterans organizing pneumonia (BOOP) is a term that became prevalent in the 1980 's to describe a distinct clinical and pathological airway disorder [28]. BOOP is characterized by the presence of connective tissue masses that form plugs within the lumen of small airways and can extend in a continuous fashion into alveolar ducts and alveoli, indicative of organizing pneumonia [28-29]. The polypoid endobronchial connective tissue masses consist 
of myxoid fibroblastic tissue as well as granulation tissue [30-31]. The intraluminal polyps have a central collection of inflammatory cells made up of histocytes, lymphocytes and plasma cells [28]. Analysis of polyp cross sections reveal the prominence of fibroblasts as well as fibrinous exudates [32]. Other major features include interstitial mononuclear cell infiltrate that are variable in density, chronic inflammation of the alveolar walls indicated by type II cell hyperplasia and reactivity, increased presence of foamy cells or macrophages in the alveolar spaces and the absence of honeycombing [33-34]. The general architecture of the lung is maintained with the polyps representing BOOP lesions. The BOOP lesions begin as focal lesions within the alveoli and terminal bronchioles of the lung and progress bilaterally over time [34]. The resulting fibrosis can be described as patchy in appearance. The alveoli appear normal except for the slight thickening of the septa resulting from inflammatory infiltrate and prominence of type II cells. Generally speaking, BOOP results in interstitial scarring as a result of inflammatory damage to airway epithelia resulting in fibroblastic interstitial thickening [35].

The development of BOOP is associated with lung injury due to environmental toxins, bacterial infections, viral infections and lung or bone marrow transplantation [29]. BOOP development can also be due to unknown etiology or more commonly known as idiopathic BOOP. Treatment for BOOP normally involves the administration of corticosteroids which have proven to be effective. However, some patients experience spontaneous remission of BOOP and over one third of patients who have completed corticosteroid treatment still have symptoms and evidence of BOOP lesions [36]. This demonstrates that not all BOOP cases respond to steroids and can be severe to fatal in some clinical cases [37]. Currently, the cellular and molecular mechanisms that mediate the formation and resolution of BOOP lesions have not been fully clarified. 


\section{Animal Model of BOOP}

Respiratory reovirus infection was demonstrated by Bellum et al. [38] as a model of BOOP. They found that CBA/J mice inoculated intranasally with $1 \times 10^{7}$ pfu of $\mathrm{T} 1 / \mathrm{L}$ reovirus demonstrated fibrous cellular plugs of the alveolar ducts characteristic of BOOP. The formation of BOOP lesions was preceded by patchy inflammation of the lungs, with focal inflammation consisting of mononuclear cell infiltrate that progressed into condensed fibrous polyps or collagenous tissue and foamy macrophages. These became well developed 3 weeks post infection. This indicated the respiratory reovirus infection can serve as an ideal model for BOOP pathogenesis and provide a means to determine the cellular and molecular mechanisms mediating disease development.

\section{Pulmonary Fibrosis}

Pulmonary fibrosis (PF) is an unremitting and progressive interstitial and diffuse parenchymal lung disease that affects 128,100 people in the United States and 5 million people worldwide [39]. The incidence is on the rise as indicated by the number of diagnoses having doubled over the past decade [39]. Roughly 40,000 people die each year due to PF, which is the same as that for breast cancer. With no effective treatments the prognosis for patients with PF is grim, with an average life span of 3-5 year after diagnosis [39]. Even though PF is extremely prevalent there are no FDA approved treatments or cures, making it one of the few remaining untreatable diseases. Advances in research and unsuccessful therapies over the past decade have revealed differing explanations of the pathogenesis of PF. Early ideas focused on the influence of chronic inflammation but immunosuppressive therapies have had limited success. Subsequently, the focus has shifted to a mechanism that involves both inflammation and repair, stating that the pathogenesis of fibrotic lung disease involves the dysregulation of normal tissue 
repair mechanisms. The loss of regulation allows for rampant fibroproliferative responses to injury and progressive development of fibrosis.

Fibrosis is an end result of a series of events that occur after damage to the epithelium and/or endothelium that results in abnormal tissue repair [40-42]. Under normal circumstances, epithelial repair in the lungs proceeds as demonstrated in figure 3 [43]. At the outset of repair, inflammatory mediators are released that activate platelet aggregation, clot formation and the formation of a provisional extracellular matrix. Subsequently, inflammatory processes ensue with the production of growth factors, cytokines and chemokines, resulting in the recruitment and proliferation of leukocytes that are responsible for the removal of dead tissue and promotion of angiogenesis. Fibroblasts transform into their active form, myofibroblasts, known for their expression of $\alpha$-smooth muscle actin ( $\alpha$-SMA) and their ability to contract. Myofibroblasts are responsible for wound contraction and closure. The final steps include epithelial and/or endothelial cell division and migration to the site in order to replace the damaged tissue. The loss of regulation in these processes results in detrimental accumulation of extracellular matrix (ECM) components, specifically the over production of collagen resulting in fibrosis. 


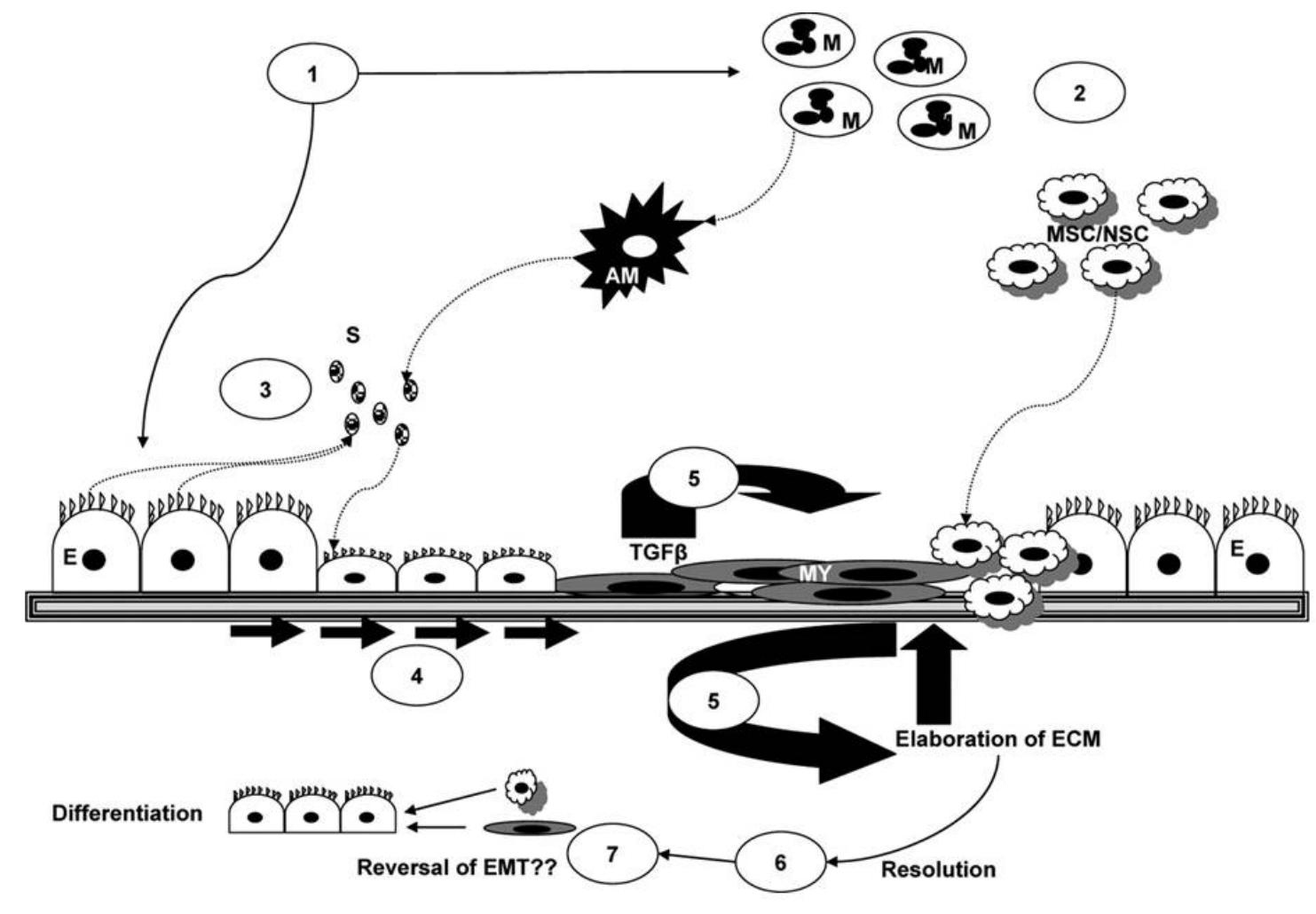

Figure 3 Epithelial Repair Process and Remodeling

(1) injury initiates activation of macrophages (M) to activated macrophages (AM) and the subsequent recruitment of immune cells, (2) mesenchymal stem cells (MSC), or niche stem cells (NSC). Soluble factors (S) secreted by immune and non immune cells (3) act on epithelial cells and fibroblasts which spread and migrate (4) closing of the wound and re-establishing intact barrier function. TGF- $\beta$ signalling results in trans-differentiation of epithelial cells to myofibroblasts (MY), the EMT, and the initiation of a chronic inflammatory response (5) with further elaboration of ECM. During resolution (6), immune cells disappear from the site of injury, macrophages are deacitivated, cells differentiate, and hyperproliferation is reduced through apoptosis, allowing tissue remodelling (7). American Journal of Physiology. Lung cellular and molecular physiology by Lynn M. Crosby \& Christopher M. Waters. Copyright 2010 by AMERICAN PHYSIOLOGICAL SOCIETY. Reproduced with permission of AMERICAN PHYSIOLOGICAL SOCIETY in the format of a Thesis via Copyright Clearance Center.

Variation in the amount and profile of cytokines and growth factors secreted during injury or inflammation serve as the primary determinants in whether or not repair is abnormal. The initiation of abnormal repair and development into fibrosis has been attributed to the mediator transforming growth factor $-\beta$ (TGF- $\beta$ ) [44-46]. It has been shown that TGF- $\beta$ is able to control the ECM transcription via a variety of signaling molecules to induce fibrosis (Figure 4) [46-48]. Specifically, TGF- $\beta$ signaling through its receptor and smad- $2 / 3$ induces increased $\alpha$ SMA expression [40, 49-51]. Alternatively, the extracellular signal-regulated kinase (ERK) signaling pathway induces collagen type 1 expression and the c-Jun-N-terminal kinase (JNK) 
pathway induces fibronectin expression [45]. Therefore, regulation of TGF- $\beta$ signaling is key in controlling the fibrotic process.
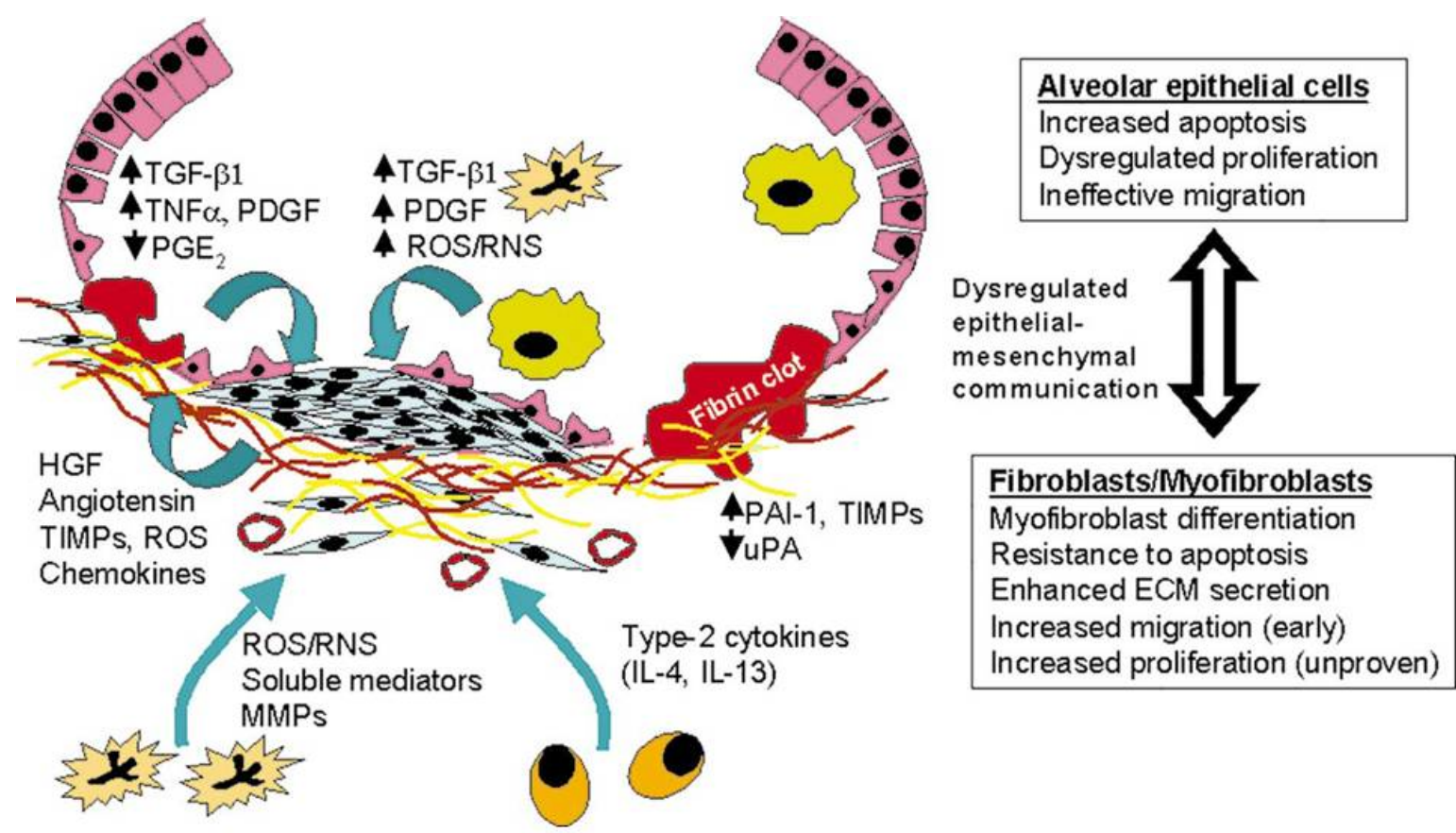

Figure 4 Altered Repair Results in Fibrosis

Epithelial-mesenchymal communication is dysregulated in progressive pulmonary fibrosis with aberrant bidirectional signaling between tissue compartments. Inflammatory cells that modulate this process are recruited and activated by secreted products from these "resident" cells. Resultant effects on alveolar epithelial cells include increased apoptosis, dysregulated proliferation, and ineffective migration. Effects on mesenchymal cells include myofibroblast differentiation, resistance to apoptosis, and enhanced extracellular matrix (ECM) secretion. Dysregulation of the repair process leads to persistent mesenchymal cell activation that culminates in pulmonary fibrosis. Abbreviations: TGF, transforming growth factor; TNF, tumor necrosis factor; PGE2,prostaglandin E2; PDGF, platelet-derived growth factor; reactive oxygen/nitrogen species, ROS/RNS; HGF, hepatocyte growth factor; TIMP, tissue inhibitors of matrix metalloproteinases; MMP, matrix metalloproteinase; PAI-1, plasminogen activator inhibitor-1; uPA, urokinase-type plasminogen activator. ANNUAL REVIEW OF MEDICINE by Victor J. Thannickal, Galen B. Toews, Eric S. White, Joseph P. Lynch III, and Fernando J. Martinez. Copyright 2001 by ANNUAL REVIEWS, INC.. Reproduced with permission of ANNUAL REVIEWS, INC. in the format Dissertation via Copyright Clearance Center.

There are many different possible and relevant mediators that are involved in repair processes that could be responsible for the development of fibrosis. The key features of airway remodeling include: the accumulation and activation of inflammatory cells, a $\mathrm{T}_{\mathrm{h}} 2$ cell immune response phenotype, mesenchymal cell hyperplasia and increased matrix protein deposition as shown in figure 4. This review focuses on the role of caveolin-1 (cav-1) and the eicosanoids in the development of fibrosis in relation to airway reovirus infection. 


\section{CHAPTER 2}

\section{Caveolae: Caveolin-1 (cav-1)}

\section{Introduction}

Caveolae (Latin for "little caves") are a subset of lipid rafts, which are described as highly-ordered microdomains within the plasma membrane [52-53]. They were first identified by electron microscopy (EM) examination in the 1950's by Palade and Yamada, who described them as $50-100 \mathrm{~nm}$ "flask shaped" membrane invaginations or flat pits that are anchored by the actin cytoskeleton [54-55] (Figure 5-A). Other distinguishable features of caveolae include their unique lipid profile that are rich in glycosphingolipids, sphingomyelin and cholesterol and by their principal protein components known as caveolin [56-59]. Caveolae are found in a vast array of terminally differentiated cell types, including endothelial cells, pneumocytes, fibroblasts, adipocytes and muscle cells [60-61]. 

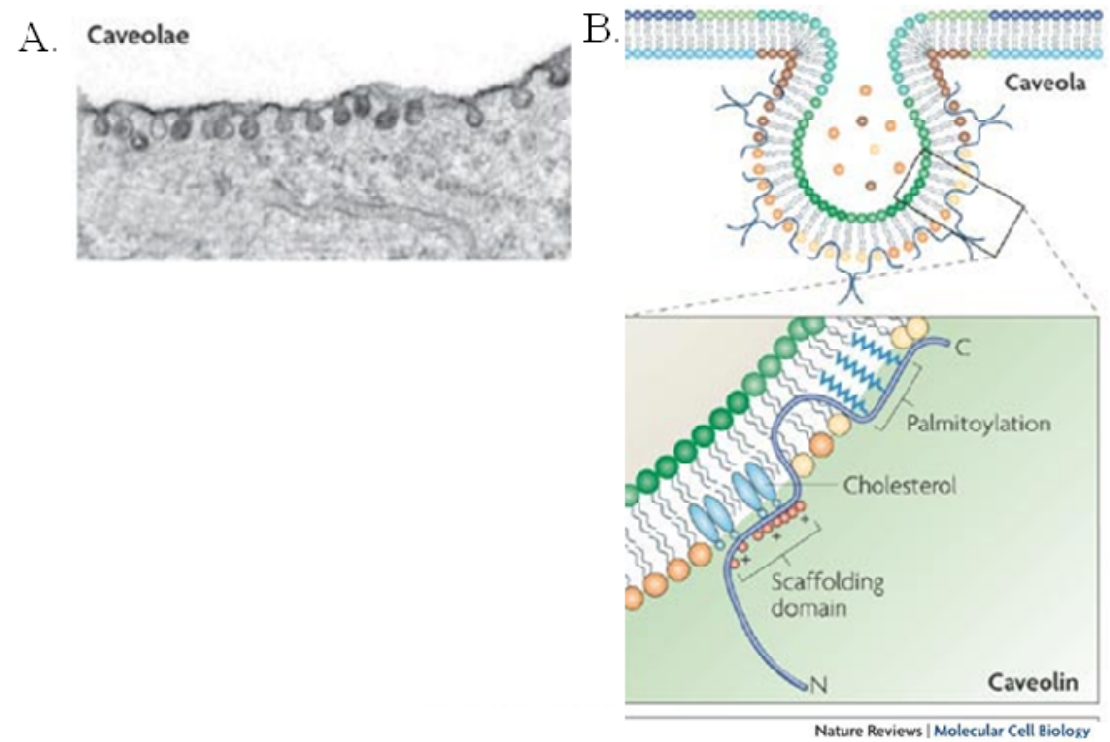

Reprinted by permissicn from Macm ilan Publshess Ltd: Nature Reviews Molecular Cell Biology, copyright 2007

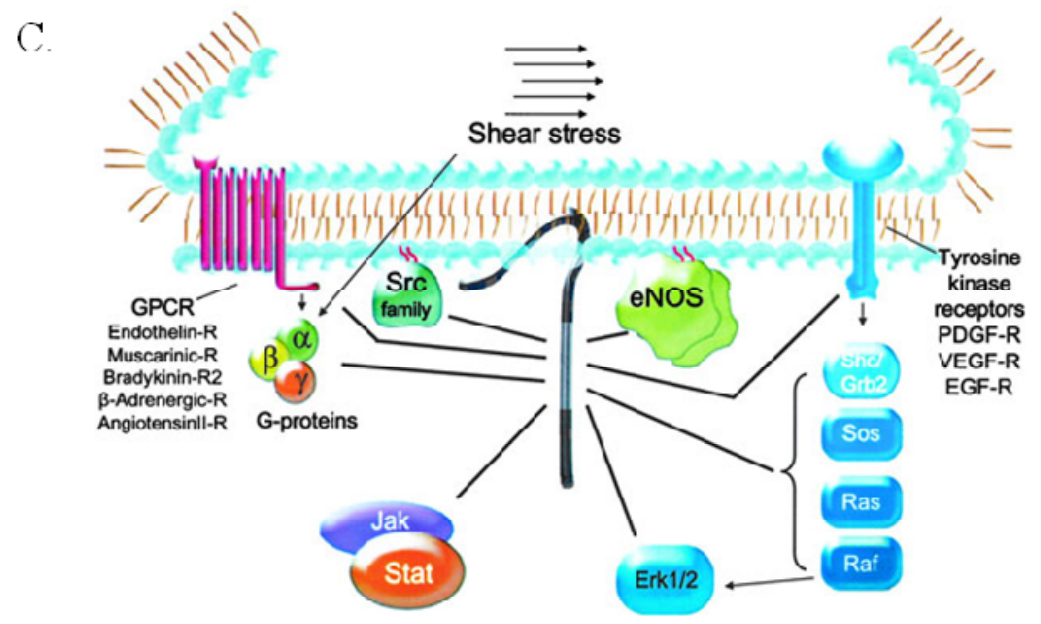

\section{Figure 5 Membrane Topology, Domains and Signaling}

A) The electoron micrograph show caveolae in adipocytes that have been surface-labeled with an electron-dense marker. B) (top) Schematic demonstrating caveolin inserted into the caveolar membrane, with the $\mathrm{N}$ and $\mathrm{C}$ termini facing the cytoplasm and the 'hairpin' intramembrane domain embedded within the membrane bilayer. The scaffolding domain has a conserved basic $(+)$ and bulky hydrophobic residues (red circles) and the $\mathrm{C}$-terminal domain, which is close to the intramembrane domain, is modified by palmitoyl groups that insert into the lipid bilayer. (bottom) The predicted membrane topology of Cav-1 as a dimer and a schematic of the cav-1 domains. Macmillan Publishers Ltd.. Reproduced with permission from Nature Publishing Group in the format of a Thesis (2010) via Copyright Clearence center. C) Regulation of signaling via caveolae microdomains. G-proteincoupled receptors (GPCR), G-proteins ( $\alpha$ and $\beta \gamma$ ), and receptor tyrosine kinases can reside in or translocate in and out of caveolae. Cav-1 directly regulates protein function or indirectly regulates Ras-Stat, extracellular signal-regulated kinases (Erk), and Janus kinases (Jak)-Stat signaling pathways. Lipid-modified proteins such as endothelial nitric oxide synthase (eNOS) and the Src family of kinases can target to caveolae and interact with caveolin-1. Reproduced with permission from Wolters Kluwer Health: Circulation Research [62], copyright 2004. 
The earliest function of caveolae was deduced based on its membrane location and the protein components present in each, which indicated that caveolae, were primarily involved in vesicle trafficking [61, 63]. For example, caveolae play a role in clathrin-independent endocytosis, transcytosis, potocytosis, cholesterol transportation and calcium metabolism [6469]. The early description of caveolae function only describes one of the two purposes of caveolae. The second function of caveolae is to regulate signal transduction, allowing them to be regarded as signalosomes $[60,70-71]$. More specifically, caveolae serve as signaling platforms by compartmentalizing and concentrating various classes of signaling molecules, including Gprotein subunits, receptor and non-receptor tyrosine kinases and other protein components (Figure 5-C) [72-76]. In summary, caveolae are involved in a variety of cellular processes including endocytosis, lipid homeostasis and signal transduction. The multifunctional nature of caveolae provides a paradigm by which numerous disease processes may be affected, indicating the importance of caveolae [77-81].

\section{Cav-1 Protein}

The integral membrane proteins, called caveolin, are the principal and indicative protein components of caveolar membranes [60]. They are predominantly located at the plasma membrane and serve as protein markers of caveolae, but are also found in the Golgi, endoplasmic reticulum (ER), in vesicles and at cytosolic locations [52, 82-83]. The caveolin gene family consists of three members in vertebrates: caveolin-1, caveolin-2 and caveolin-3, which encode four protein isoforms (caveolin-1 $\alpha$, caveoline-1 $\beta$, caveolin-2 and caveolin-3) [60].

Caveolin-1, also known as Cav-1 or VIP21, is a 21 to $24 \mathrm{kDa}$ protein that associates with the cytoplasmic surface of caveolae. It was the original member of this family and serves as the primary structural component of caveolae [84] (Figure 5-A). In fact, along with cholesterol and 
sphingolipids, cav-1 appears to play a direct role in caveolar biogenesis $[57,60,85]$. The two other members of the caveolin family, caveolin-2 (cav-2) and caveolin-3 (cav-3), also target exclusively the caveolar microdomains [60]. The expression levels of cav-1, -2 , and -3 fully correlate with the presence of caveolae in those tissues. Interestingly, the expression patterns of cav-1 and -2 are largely distinct from that of cav-3; whereas adipocytes, endothelial cells, pneumocytes, and fibroblasts have the highest expression levels of cav-1 and -2 , cav-3 expression is limited to muscle cells of all types (cardiac, skeletal, and smooth muscle cells) [52, 86-87]. The three proteins overlap in their expression in a few cell types, including smooth muscle cells. Since their discovery, the caveolin proteins have been shown to be intimately involved in all aspects of caveolar function.

The many functions of caveolae can be attributed to its major protein component, cav-1. Cav-1 is able to interact with signaling molecules, scaffolding proteins and different membrane components through the different domains found along its structure (Figure 5-B). The 3 distinct structural regions of cav-1 are the hydrophilic cytosolic N-terminal (1-101 residues) and Cterminal (135-178 residues) regions which flank the central hydrophobic domain(102-134 residues) creating a hairpin-like structure that spans the plasma membrane [60]. The N-terminal and C-terminal are oriented towards the cytosol with the central region located in the membrane. The membrane attachment domains are located at the N- and C-termini and are designated as NMAD (residues 82-101) and C-MAD (residues 135-150) [88-89]. Located along the c-terminal are palmitoylation sites on cysteine (Cys) 133, 143 and 156, that are involved in membrane anchorage [90-91]. The central region (residues 102-134) was first suggested to be a transmembrane domain (TMD), but after predicting its beta-sheet rather than alpha-helix conformation, it was suggested that it was a protein binding domain. Further analysis indicated 
that the domain was involved in hetero-oligomerization of cav-1 with cav- 2 and in specific interactions with other proteins [92]. The caveolin scaffolding domain (CSD), located at the Nterminus (amino acids 82-101), is involved in the binding and inhibition of proteins containing a defined caveolin binding motif, such as $\omega \operatorname{xxx} \omega x \operatorname{xx} \omega$ or $\omega \operatorname{x} \omega \operatorname{xxxx} \omega$ - where $\omega$ is an aromatic amino acid (Trp, Phe or Tyr) [93]. The oligomerization domain (amino acids 61-101) contains CSD and directs the formation of homooligomers (14-16 cav-1 molecules), which interact with cholesterol and signaling molecules [89].

The organization of the individual cav-1 domains provides a structural platform for recruiting and compartmentalizing signaling proteins (Figure 5-C). Several signaling proteins have been reported to assemble in caveolae and interact with cav-1 such as: receptor tyrosine kinases and their downstream associates (Epidermal Growth Factor Receptor (EGFR), Platelet Derived Growth Factor Receptor (PDGFR), v-Ha-ras Harvey rat sarcoma viral oncogene homolog (H-Ras), V-raf-1 murine leukemia viral oncogene homolog 1 (Raf-1), ERK, Phosphatidylinositol 3-kinases (PI3K)) [94-100], non-receptor tyrosine kinases (Src family, Fak) [99, 101], receptor serine/threonine kinases (TGF- $\beta$ receptor type I) [102-103], G-protein coupled receptors and their downstream associates (G-protein alpha subunits, adenylyl cyclase, Protein Kinase-A (PKA), PKC isoforms) [104-108], steroid hormone receptors (Androgen Receptors (AR) and Estrogen Receptors (EstR)) [109-111], Endothelial nitric oxide synthase (eNOS) [112-114] and numerous others. Cav-1 binding acts as a negative regulator upon most signaling molecules. For example, as a tumor suppressor, cav-1 binds to EGFR and inhibits phosphorylation and activation of the receptor. Cav-1 also is able inhibit activation by binding directly to signaling molecules and stopping signaling cascades. 


\section{Cav-1 and Inflammation}

The involvement of cav-1 in the processes of inflammation is multifaceted and is indicative of its varied functions as a membrane protein. The expression of cav-1 by immune cells is not well defined due to varying results from different cell lines. Previous research suggests that it is expressed by myeloid, but not lymphoid cells, but due to more specific and elaborate detection methods, recent evidence suggests that it is present in all immune cells. Cav1 has been identified in murine macrophages [115] and mast cells [116], human dendritic cells [117], mast cells (MCs) [116], lymphocytes [85] and neutrophils (PMNs) [118] and bovine monocytes, macrophages and dendritic cells (DCs) [119]. Cav-1 expression in these cells is not isolated to the cell surface, but can be present intracellularly as in the case of bovine DCs where cav-1 is focally isolated to the perinuclear region. Expression may also not be constitutive but inducible by different signals, making it difficult to determine the expression. This is the case with peritoneal macrophages, which demonstrate sparse caveolin staining concentrated in vesicles in the golgi region. Subsequent stimulation with Freund's adjuvant results in increased caveolin staining that is no longer restricted to the golgi, but dispersed throughout the macrophage. Murine macrophages demonstrated similar increases in cav-1 post exposure to lipopolysaccharide (LPS). Therefore, cav-1 expression may not be constitutive, but rather transient and stimulus specific. The nature of cav-1 expression amongst immune cells is dependent on different factors including cell type and state of activation, indicating that cav-1

could be an important component in mediating specific immune cell functions. It has been demonstrated that cav-1 plays a part in regulating PMN, macrophage, antigen presenting cell (APC), B cell and $\mathrm{T}$ cell functions. 
The presence and function of cav-1 in PMNs is incompletely understood. Studies have indicated that cav-1 is important in the mechanism of PMN activation, adhesion and transmigration [120]. PMN activation induces the formation of the primary source of oxygen derived reactive species, superoxide. Hu et al. [120] observed that the production of superoxide in response to stimulus with PMN activating factors formyl-Met-Leu-Phe (fMLP) plus plateletactivating factor (PAF) is reduced by $50-80 \%$ in cav- ${ }^{-/-}$PMNs compared to control, indicating cav-1 mediates PMN activation. Furthermore, cav-1 ${ }^{-/-}$PMN migration and adhesion to fibrinogen in response fMLP is reduced compared to controls. Similarly, both Hu et al. [120] and Garrean et al. [121] observed that activated cav- $1^{-1-}$ PMNs exhibited deficient adherence and migration to and across pulmonary micro vascular endothelial cell monolayers compared to controls. Combined, this indicates that cav-1 is essential for PMN adhesion and migration.

Macrophages are one of the primary host immune defense cells, playing an essential role in phagocytosis, cytokine production and antigen presentation. Cav-1 has been shown to be an important regulator of macrophages during inflammation, regulating macrophage apoptosis [122], lipid metabolism [123], endocytosis [124] and immunomodulatory effects [125]. The role of cav-1 in macrophage apoptosis was elucidated by studies using thioglycollate-elicited mouse peritoneal macrophages (tg-MPM). tgMPMs treated with different apoptotic agents, including simvastatin, camptothecin or glucose deprivation, resulted in large increases of cav-1 expression [122]. Cav-1 increase was determined to be a specific marker of apoptosis that is closely associated with the externalization of phosphatidylserine (PS) [122]. Cav-2 did not show similar changes in response to the apoptotic agents, indicating this was specific to cav-1.

Cav-1 is important in mediating macrophage lipid metabolism [123]. Specific characteristics of cav-1, such as its ability to bind to cholesterol and it location in cholesterol rich 
lipid rafts, imply its involvement in the maintenance of cellular cholesterol homeostasis or cholesterol efflux. Specifically, caveolae and cav-1 are associated with the proteins CD36 and SR-BI, two members of the B scavenger receptor family that are responsible for high density lipid (HDL) transfer. Studies have shown that cav-1 regulates the activity of both receptors, altering cholesterol efflux [123].

The relationship between cav-1 and macrophages is mainly focused on uptake of antigen since cav-1 is essential for non-clathrin coated endocytosis [126-127]. Cav-1 deficient macrophages demonstrated impaired phagocytosis that was determined to be dependent upon cav-1 expression [124]. Also, treatment with the caveolae inhibitor filipin decreased the speed of fluid phase and receptor mediated endocytosis by macrophages[127]. Combined, these two studies indicate the importance of cav-1 in phagocytosis by macrophages.

Cav-1 has been shown to be a potent immunomodulatory molecule in macrophages. Macrophage expression of cav-1 is not consistent for all subtypes, but is dependent upon their location, environmental signals and function. Recently, it was verified that cav-1 is expressed by peritoneal macrophages (PMs) by Kiss et al. [128]. It was also reported that cav-1 expression by PMs is intimately involved in the processes of inflammation and pathogenesis of atherosclerosis [123]. Similar to PM, the expression of cav-1 in alveolar macrophages (AMs) was undetermined until recently when Wang et al [125] observed cav-1 expression in murine AMs by western blotting and immunofluoresence microscopy. The importance of cav-1 expression by AMs and PMs was determined by loss of function analysis using small interfering RNA (siRNA) to down regulate cav-1 expression. Down regulation of cav-1 increased PM and AM inflammatory response to LPS stimulation, with increased tumor necrosis factor- $\alpha$ (TNF- $\alpha$ ) and IL-6 production. However, it also decreased the production of the anti-inflammatory mediator IL-10. 
Furthermore, over expression of cav-1 in a murine macrophage cell line demonstrated a significant attenuation of the proinflammatory cytokine TNF- $\alpha$ and IL-6, and increased IL-10 production in response to LPS stimulus. Combined, these results indicate that cav-1 has a protective role in inflammation by suppression of proinflammatory cytokine (TNF- $\alpha$ and IL-6) production and augmentation of anti-inflammatory cytokine (IL-10) production in response to LPS.

Results from studies of cav-1 and the adaptive immune response has provided evidence that cav-1 regulates both T cell and B cell activity. A study by Ohnumua et al. [129] showed that cav-1 is the co stimulatory ligand for CD26, and that interaction/ligation by cav-1 results in $\mathrm{T}$ cell proliferation and activation with co stimulation from T cell Receptor (TCR)/CD3. Similarly, cav-1 has been shown to influence B cell development of thymus independent immune responses [130]. Studies of cav- $1^{-/-}$mice's humoral response to thymus dependent and independent antigens demonstrated decreased immunoglobulin production in response to thymus independent antigen. This demonstrated that cav-1 deficient mice are unable to develop a normal thymus independent immune response, implicating that B cell thymus independent immunity is regulated by cav-1. Therefore, cav-1 plays an integral part in regulating aspects of the adaptive immune response.

Cav-1 serves as a central molecule in signaling cascades allowing it to regulate multiple signaling pathways. Functioning as a scaffolding protein, cav-1 organizes and concentrates various classes of signaling molecules, including G protein subunits, receptor and non-receptor tyrosine kinases and small GTPases within caveolae. Moreover, cav-1 also inhibits the activation of downstream signaling events of many proteins, including c-Src, H-Ras, mitogen activated protein (MAP) kinases. The primary example of cav-1 regulation of inflammatory 
signaling is the interaction between cav-1 and nitric oxide (NO) production. Cav-1 inhibition of eNOS regulates inflammatory signaling by decreasing NO production which inhibits the activation of nuclear factor kappa-light-chain-enhancer of activated B cells (NF-kB) and synthesis of proinflammatory proteins [121]. Another important inflammatory signaling mediator, cyclooxygenase-2 (Cox-2), has recently been reported to be regulated by cav-1, but by a different molecular mechanism [131]. In the ER, soluble cav-1 binds to Cox-2 and facilitates its degradation, suppressing Cox-2 mediated proinflammatory activation. Combined these two examples demonstrate that cav-1 is a critical regulator of inflammatory signaling cascades and key in the processes of inflammation.

\section{Cav-1 and Fibrosis}

There is extensive evidence associating cav-1 with the regulation of collagen expression in vitro and in vivo. Experimental models of fibrosis show evidence of decreased cav-1 expression compared to controls. A good example is the decrease of cav-1 expression in bleomycin-induced fibrosis tissue as well as in cell cultures [132]. Wang et al. observed a twofold reduction in cav-1 mRNA in IPF lung tissues compared to control [133]. Cav-1 protein expression in idiopathic pulmonary fibrosis (IPF) lung tissue was decreased by $73 \%$ when compared to controls [133]. The association between reduced cav-1 expression and collagen over expression in fibrotic tissue is further substantiated by the fibrotic phenotype seen in cav-1 knockout mice [134-135]. It is apparent that cav-1 and collagen expression are inversely related indicating that cav-1 works in opposition of profibrogenic processes. Profibrogenic processes are regulated by several mediators, such as $\mathrm{T}_{\mathrm{h}} 2$ cytokines and TGF- $\beta$, and different cell types, specifically myofibroblasts. Each of these mediators and cell types are regulated by cav-1 and are fundamental in understanding the pathogenesis of fibrosis. 
Cav-1 serves as a signaling platform for numerous signaling molecules. The interaction of kinases with cav-1 inhibits their activity and therefore prevents profibrotic signaling. A primary example is cav-1 suppression of TGF- $\beta$ profibrotic signaling (Figure 6). The regulation of TGF- $\beta$ signaling involves numerous signaling pathways allowing for different methods to inhibit activation. TGF- $\beta$ SMAD2/3 phosphorylation and nuclear translocation is responsible for activating fibroblast $\alpha$-SMA expression, fibronectin production and collagen type I production. The TGF- $\beta$ receptors (TGF- $\beta$ Rs) I and II are localized in cav-1 enriched fractions of human endothelial cells and have been reported to interact by immunopercipitation. The interaction between cav-1 and TGF- $\beta$ RI was reported by Razani et al. to inhibit smad- 2 phosphorylation, preventing the smad complex formation and nuclear translocation in fibroblasts [102]. TGF- $\beta$ induced ERK1/2 signaling regulates collagen type I production [45]. ERK1/2 have been demonstrated by Mineo et al. [136] to localize to caveolae and directly interact with cav-1. Interaction between cav-1 and ERK1/2 inhibits collagen type 1 production. JNK activation is pivotal for TGF- $\beta$ induced fibronectin production and is regulated by cav- 1 via modulation of SMAD2 phosphorylation and nuclear translocation. Therefore, cav-1 regulates TGF- $\beta$ profibrotic signaling by suppressing each signaling cascade and preventing profibrotic signals. 
A.

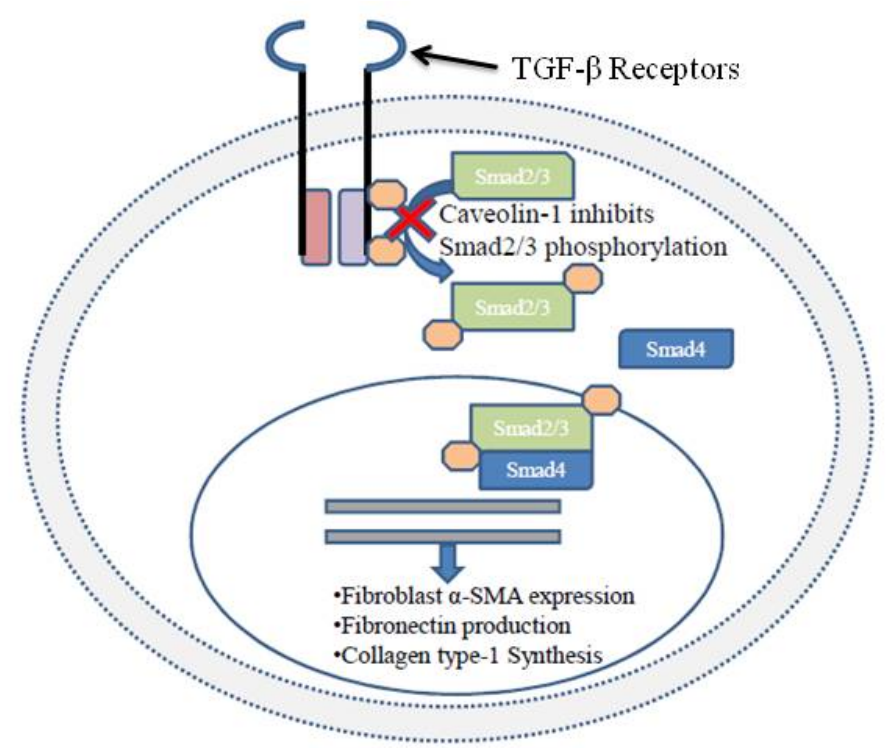

B.

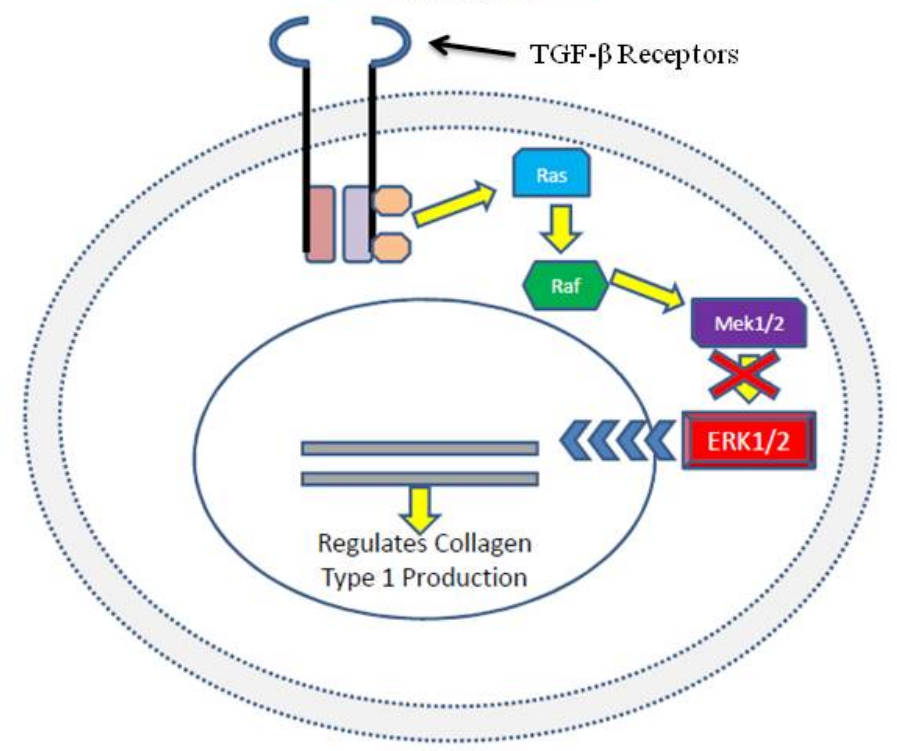

Figure 6 TGF- $\beta$ Profibrogenic Signaling

A) Diagram that illustrates fibrogenic TGF- $\beta$ signaling pathways. After TGF- $\beta$ binding, Type II receptors (T $\beta$ R-II), which are constitutively active serine-threonine kinases, recruit and dimerize with type I receptors (T $\beta R-I)$. Subsequently, T $\beta R-I I$ phosphorylates the T $\beta R$-I, resulting in their activation. Active T $\beta R-I$ specifically phosphorylates receptor-regulated Smad 2 and Smad3 via the protein kinase ALK-5. Phosphorylated Smad 2 and Smad 3 then complex with co-Smad4, resulting in their transport to the nucleus. In the nucleus, they cooperate with other factors to regulate transcription of critical genes, here represented by genes encoding CTGF and $\alpha 1$ and $\alpha 2$ type I collagens. B) After TGF- $\beta$ binding, Type II receptors (T $\beta$ R-II), which are constitutively active serine-threonine kinases, recruit and dimerize with type I receptors (T $\beta R-I)$. Subsequently, T $\beta R-I I$ phosphorylates the T $\beta$ R-I, resulting in their activation. T $\beta$ R-I results in activation of the Ras-Raf-Erk signaling pathway which culminates in the production of type-1 collagen. 


\section{CHAPTER 3}

\section{Eicosanoids}

\section{Introduction}

The eicosanoid family of lipids are integral mediators of inflammation and homeostasis that are derived from a single membrane released 20 carbon fatty acid, arachidonic acid [137]. These lipid mediators have numerous functions that include roles in inflammation, fever, regulation of blood pressure, blood clotting, immune system modulation, control of reproductive processes, tissue growth and regulation of sleep/wake cycle [138]. Members of the eicosanoid family include prostaglandins (PGs), leukotrienes (LTs), prostacyclins, thromboxanes $\left(\mathrm{TXA}_{2}\right)$ and epoxyeicosatrienoic acids (EETs). They can be classified by their biological activity into two categories; those that promote inflammation (pro-inflammatory) and tissue destruction and those that stop inflammation (anti-inflammatory) and promote tissue repair [137]. The synthesis and degradation of the eicosanoids is tightly regulated to allow the proper balance of damage and repair and to prevent an imbalance or disease resulting from either chronic inflammation or abnormal fibropriliferation [139]. Therefore, the interaction and balance between eicosanoids is critical to homeostasis and could provide insight into the pathology of inflammatory and fibrotic disease.

The pathobiology of pulmonary fibrosis can be attributed to the aberrant fibroproliferative response to lung injury resulting from the loss of regulation over tissue repair processes. This results in the remodeling of the airway that is characterized by the loss of alveolar epithelial cells that are replaced by excessive deposition of matrix proteins such as collagen. Eicosanoids regulate the cells and pathways involved in the inflammatory response and tissue repair and play a significant role in the pathology of lung fibrosis. PGS and LTs are 
the two eicosanoid metabolites whose biological activity has been implicated in the pathology of lung fibrosis [140]. Increased levels of LTs and decreased levels of PGs are a characteristic of profibrotic milieus. Studies have indicated that LTs, specifically cysteinyl leukotrienes (cysLTs), promote predominantly proinflammatory and fibroproliferative activity, while PGs predominantly do the opposite [139]. This review will focus on PGs and LTs and their role in pulmonary fibrosis.

\section{Initiation of Synthesis:}

The initiating step in the synthesis of eicosanoids is the release of arachidonic acid from membrane phospholipids by cytosolic phospholipase A2 (cPLA2). Arachidonic acid is stored in abundance as an esterfied component of the phospholipid bilayer and whose release is tightly regulated by the activity of type IV cPLA2 [141]. Optimal functional activation of this enzyme requires intracellular calcium flux and phosphorylation by mitogen-activated protein kinases (MAPKs) which is inhibited by anti-inflammatory signaling such as corticosteroids and Aspirin [142]. Thus, the onset of an inflammatory response is associated with a surge in production of these lipid mediators. Cells are activated by mechanical trauma or by specific cytokines, growth factors or other stimuli such as the inflammatory signal platelet activating factor or bradykinin. Stimulus results in the activation and translocation of cPLA2 to the nuclear envelope, ER and golgi apparatus [143]. Once activated cPLA2 hydrolyzes the ester linkage between a fatty acid and the $\mathrm{OH}$ at the $\mathrm{C} 2$ of the glycerol backbone, releasing inositol trisphosphate (IP3) and diacylglycerol which is then catalyzed by diacylglycerol lipase into arachidonic acid. Free arachidonic acid can then be metabolized into any eicosanoid lipid mediators based on the abundance and activity of the specific synthetic enzymes. 


\section{Prostaglandins (PGs) Synthesis}

Cyclooxygenase-1 and cyclooxygenase-2 (COX-1 and COX-2), more correctly termed prostaglandin endoperoxide H synthases- 1 and -2 (PGHS-1 and PGHS-2), are key enzymes that catalyze the first committed step in the synthesis of PGS from fatty acid precursors [144] (Figure 7). COX-1 is always present in tissues and is responsible for basal and constitutive PG synthesis, while COX-2 is induced by appropriate physiological stimuli (cytokines, tumor promoters and growth factors) in various inflammatory or "induced" settings [145-146]. At the ER and nuclear membrane arachidonic acid is presented to $\mathrm{COX}-1 / \mathrm{COX}-2$ and is metabolized to the intermediate prostaglandin $\mathrm{H}_{2}\left(\mathrm{PGH}_{2}\right)$. A range of $\mathrm{PGs}$ are generated by conversion of $\mathrm{PGH}_{2}$ by specific terminal synthases that are cell- and tissue-specific.

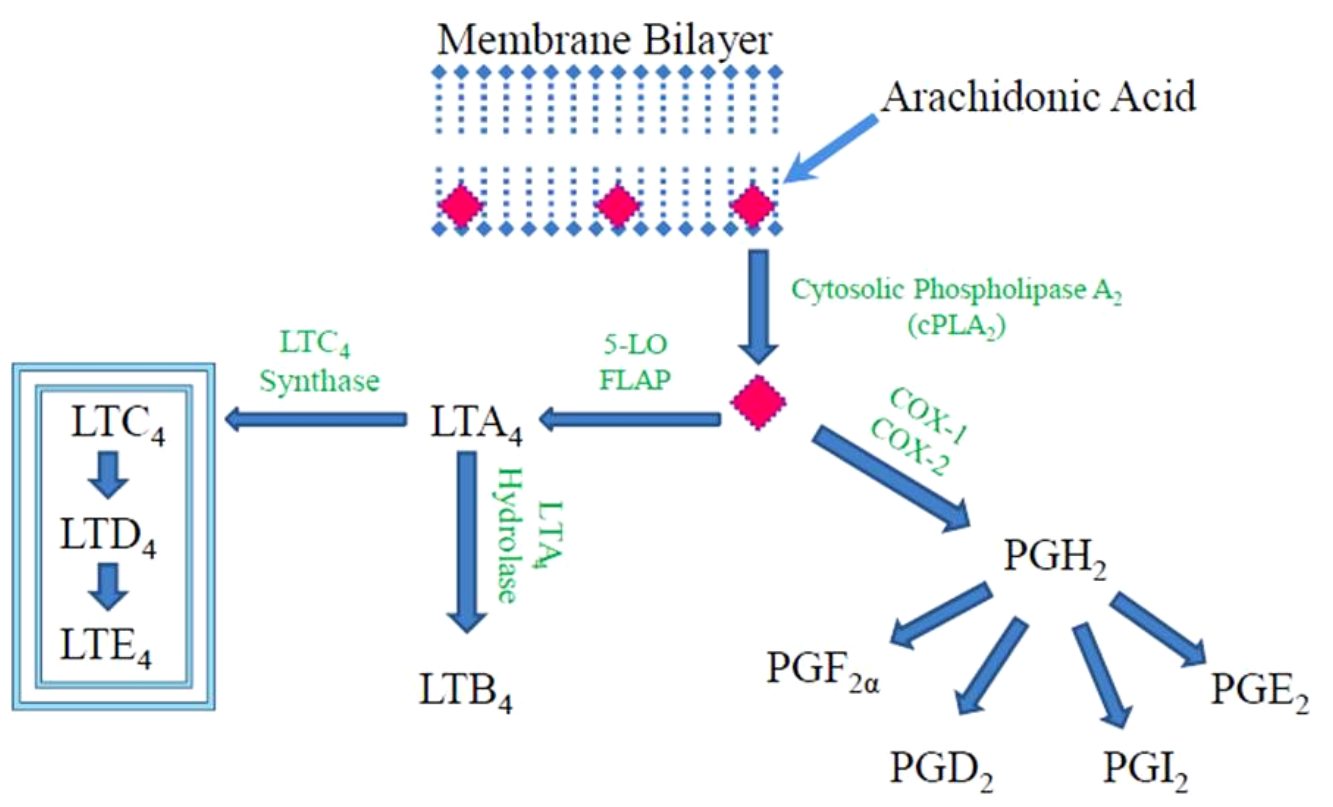

Figure 7 Eicosanoid Synthesis

Cytosolic phospholipase A2 (cPLA2) releases arachidonic acid from the membrane phospholipids that is metabolized by either 5lipoxygenase (5-LO) to initiate the production of the leukotrienes or by cycloxygenase $-1 / 2$ to initiate the production of the prostaglandins. The synthesis of specific eicoasnoids is carried out by terminal synthases. 
The pairing of $\mathrm{PGH}_{2}$ synthesis and production of downstream PGs by downstream enzymes and specific cell types is the primary means of regulating the production of each mediator. $\mathrm{PGE}_{2}, \mathrm{PGI}_{2}, \mathrm{PGD}_{2}, \mathrm{PGF}_{2 \alpha}$ and $\mathrm{TXA}_{2}$ are synthesized by both leukocytes and structural cells and effect these cells and local tissue. Generally speaking, $\mathrm{PGF}_{2 \alpha}$ synthase is mainly found in the uterus, prostacyclin synthase is dominantly located in endothelial cells and two types of PGD synthase are found in the brain and mast cells. Looking more specifically at the lungs, there are two PGs that are dominantly involved: $\mathrm{PGE}_{2}$ and $\mathrm{PGI}_{2} . \mathrm{PGE}_{2}$ is the most ubiquitous and abundant PG with both proinflammatory and anti-inflammatory functions dependent on context. $\mathrm{PGE}_{2}$ can be produced by most cell types that express cytosolic $\mathrm{PGE}_{2}$ synthase. More specialized cells, such as macrophages and bronchial epithelial cells express microsomal PGE synthase-1 (mPGES-1) and microsomal PGE synthase -2 (mPGES-2) [147]. mPGES are members of the microsome-assocaited proteins involved in eicosanoid and glutathione metabolism (MAPEG) that also include other enzymes involved in eicosanoid synthesis [148]. The expression of mPGES-1 and -2 is up regulated by the same signals associated with up regulation of COX-2, indicating their activity is associated with increases in inflammation. $\mathrm{PGI}_{2}$ is synthesized from $\mathrm{PGH}_{2}$ by PGI synthase (PGIS) which is expressed by endothelial cells as well as vascular and smooth muscle cells of the airway. It is highly transcribed in the heart, lungs, smooth muscle, kidneys and ovaries. It is easy to see that coordinate induction of multiple enzymes in the prostanoid pathway, in particular mPGES and COX-2, can be modified in accordance with the requirements of context to produce the appropriate PG response. The intricate interaction between these mediators is complex and can lead to the amplification or dampening of an inflammatory response and the nature of subsequent responses. 


\section{Mechanism of PG Signaling: Receptors and Cellular Effects}

PGs are mainly released through facilitated transport via the PG transporter (PGT) to interact with local cell types and tissues expressing the appropriate 7 transmembrane G protein coupled receptors (GPCR). There are at least 9 known PG receptors found in both mouse and man, along with numerous splice variants (Figure 8). To be more specific there are four specific GPCRs: $\mathrm{EP}_{1}, \mathrm{EP}_{2}, \mathrm{EP}_{3}$ and $\mathrm{EP}_{4}$ that bind $\mathrm{PGE}_{2}$, two bind PGD: $\mathrm{DP}_{1}$ and $\mathrm{DP}_{2}$ [149-152]. The PG receptors are classified into three groups among the GPCR super family based on homology and signaling attributes, with $\mathrm{DP}_{2}$ being the only exception. $\mathrm{DP}_{1}, \mathrm{EP}_{2}$, and $\mathrm{EP}_{4}$ are considered "the relaxant" receptors. This group signals through $G_{s}$ mediated increases in cyclic adenosine monophosphate (cAMP) and induce muscle relaxation and vasodilation [137]. Another group, known as the "contractile" receptors includes $\mathrm{EP}_{1}$ and signal through $\mathrm{G}_{\mathrm{q}}$ mediated increases in intracellular calcium leading muscle contraction and vasoconstriction [137]. The $\mathrm{EP}_{3}$ receptor is considered an "inhibitory" receptor and signals through $\mathrm{G}_{\mathrm{i}}$ mediated decreases in cAMP and has been known to induce fever [137]. These receptors are predominantly located at the plasma membrane in sites adjacent to PG synthesis where they can induce specific biological effects shown in figure 9. 
A.

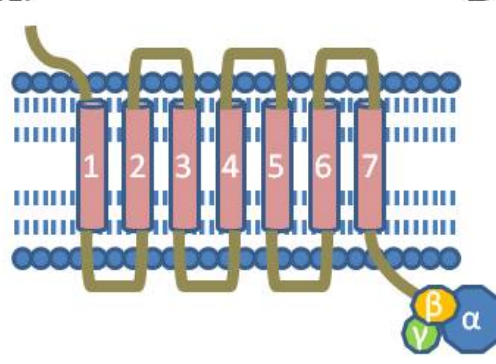

B.

Leukotriene Receptors

\begin{tabular}{|c|c|c|}
\hline Receptors & Ligands & Effect \\
\hline $\mathrm{BLT}_{1}$ & $\mathrm{LTB}_{4}$ & $\begin{array}{l}\text { - Induces Chemotaxis } \\
\text { - Inflammation } \\
\text { - Contraction }\end{array}$ \\
\hline $\mathrm{BLT}_{2}$ & $\mathrm{LTB}_{4}$ & •???? \\
\hline CysLT $_{1}$ & $\mathrm{LTC}_{4}, \mathrm{LTD}_{4}, \mathrm{LTE}_{4}$ & $\begin{array}{l}\text {-Bronchoconstriction } \\
\text {-Inflammation } \\
\text {-Edema }\end{array}$ \\
\hline $\mathrm{CysLT}_{2}$ & $\mathrm{LTC}_{4}, \mathrm{LTD}_{4}, \mathrm{LTE}_{4}$ & $\begin{array}{l}\text {-Inflammation } \\
\text {-Altered Vascular Permeability } \\
\text {-Induction of Tissue Fibrosis }\end{array}$ \\
\hline
\end{tabular}

C.

\section{Prostaglandin Receptors}

\begin{tabular}{cccc}
\hline & G Protein & Signaling & Effect \\
\hline EP1 & $G_{\mathrm{q}}$ & TCa & Contractile \\
EP2 & $G_{5}$ & TCAMP & Relaxant \\
EP3 & $G_{i}$ & ICAMP & Inhibitory \\
EP4 & $G_{5}$ & TCAMP & Relaxant \\
\hline
\end{tabular}

\section{Figure 8 LT and PG Receptors}

A) Schematic representation of a 7-transmembrane g protein coupled receptor. B) Table describing the Leukotriene receptors' ligand specificity and biological effects. C)Table describing the Prostaglandin receptors' biological effects and signaling mechanisms. 


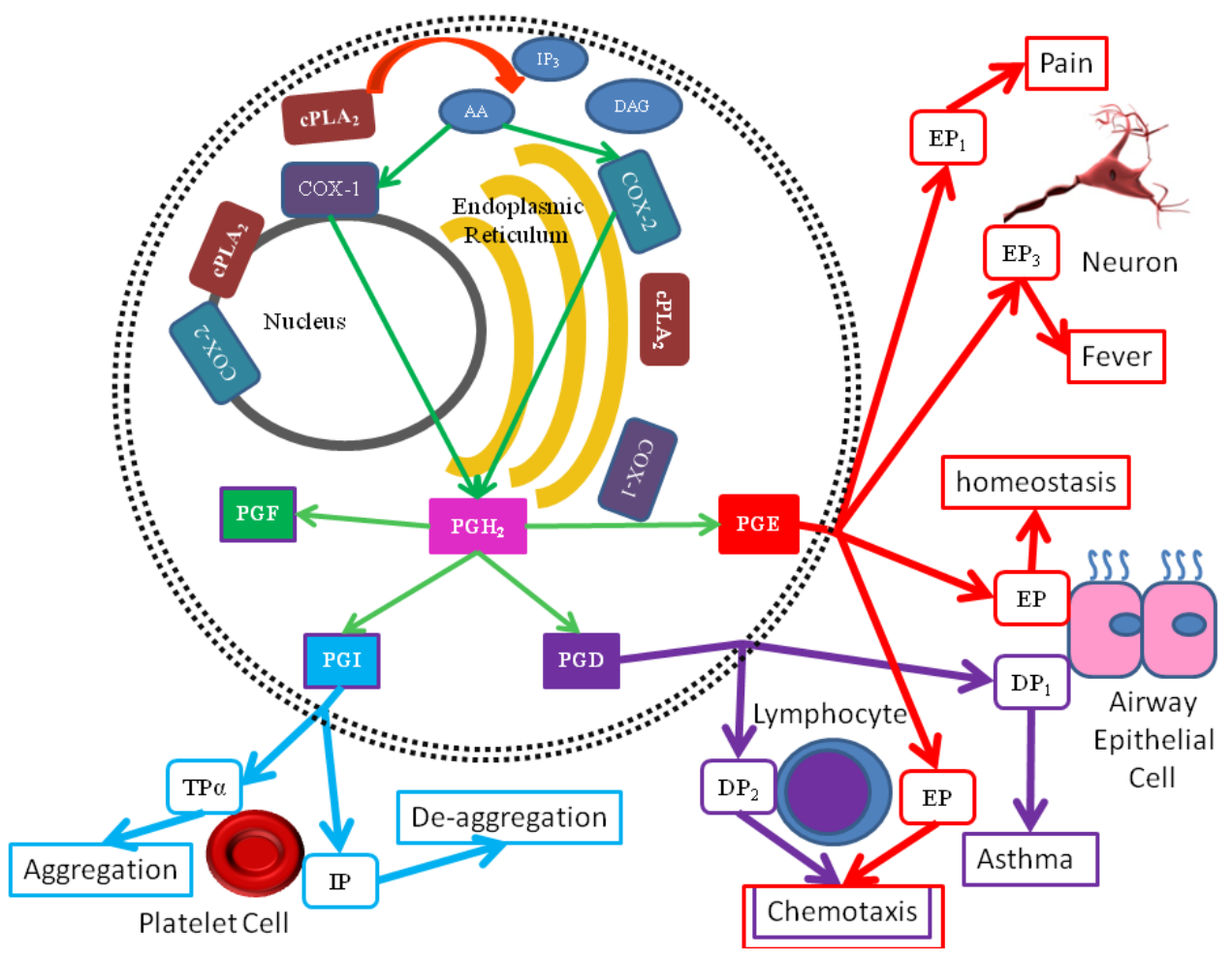

Figure 9 PG Activity

A schematic presentation of prostaglandin synthesis, secretion, receptor binding and biological activity. Upon synthesis prostaglandins can undergo facilitated transport from the cell through the prostaglandin transporter (PGT) or other carrier to exert autocrine or paracrine actions on a family of prostaglandin receptors EP1, EP2, EP3, EP4, DP1, DP2, and TP $\alpha$, on the cell types indicated. Only a few of the diverse activities of prostaglandins are shown here.

\section{The Role of PGs in Lung Inflammation}

\section{PGE2 and Its Receptors}

$\mathrm{PGE}_{2}$ is one of the best known and well studied $\mathrm{PG}$, whose diverse regulatory actions demonstrate the dual nature of this group. $\mathrm{PGE}_{2}$ influences almost every cell type involved in inflammation by acting through specific EP receptors to induce a particular response. The response to $\mathrm{PGE}_{2}$ is dependent upon which receptor is predominantly expressed; indicating that the diverse effects of $\mathrm{PGE}_{2}$ are not specific to the mediator itself, but rather is determined by the receptor. This allows for a single mediator to induce numerous sundry responses in one local area in order to produce a specific inflammatory context. 
The effects of $\mathrm{PGE}_{2}$ on individual cells of the immune system are distinct and contingent upon the function and location of the immune cell and its context, whether it is involved in the innate or adaptive wing of the immune response. This can be demonstrated by the varied effects of $\mathrm{PGE}_{2}$ signaling on different innate inflammatory cells such as mast cells (MCs). MCs have been shown to be crucial for optimal immune responses during infection because of their ability to instantly release several pro-inflammatory mediators from intracellular stores and their location at the host-environment interface during the innate response. They exert these effects by altering the inflammatory environment after detection of a pathogen and by mobilizing various immune cells to the site of infection and to draining lymph nodes. Studies have demonstrated that $\mathrm{PGE}_{2}$ acts as both an inducer and suppressor of $\mathrm{MC}$ activation by blocking the high affinity Fc receptor for IgE (FceRI) [153-154]. FceRI is a member of the multi chain immune recognition receptor family that is expressed on the cell surface of macrophages, as well as basophils, eosinophils and MCs. Cross-linking of FceRI via IgE bound multivalent antigens leads to the induction of a signaling cascade that regulates the rapid release of preformed mediators such as histamine and heparin ('degranulation'), the rapid synthesis of arachidonic acid metabolites, such as prostaglandins and leukotrienes, and the expression of cytokines (e.g. IL-3, IL-4, IL-5, IL-6, IL-10, IL-13, TNF- $\alpha$ ) and chemokines [155-157]. Inhibition of FceRI by $\mathrm{PGE}_{2}$ in MCs is attributed to high levels of cAMP and has been shown to stop exocytosis of granules, inhibit $\mathrm{LTC}_{4}$ and $\mathrm{PGD}_{2}$ generation and suppress TNF- $\alpha$. Specifically, signaling by $\mathrm{PGE}_{2}$ through the cAMP mediated $\mathrm{EP}_{2}$ receptor has been shown to block the activity of the high affinity FceRI [158-160]. This results in the suppression of FceRI mediated exocytosis and eicosanoid production. In contrast, $\mathrm{PGE}_{2}$ signaling through $\mathrm{EP}_{3}$ induces exocytosis and eicosanoid generation [159]. The suppressive effects of $\mathrm{PGE}_{2}$ on FceRI also effects $\mathrm{MC}$ 
transcription of TNF- $\alpha$ by inhibiting FceRI mediated production through the expression of the protein inducible cAMP early repressor (ICER), that can then inhibit cytokine transcription [161]. Therefore, $\mathrm{PGE}_{2}$ has the ability to regulate the activation and function of MCs, which in turn can directly determine the outcome of an inflammatory response.

$\mathrm{PGE}_{2}$ is involved in the adaptive immune response as implicated by the expression of the EP receptors on T cell, B cells and DCs. The initiation of the adaptive immune response is dependent upon the activation of APCs, specifically DCs. DCs serve as the source of stimulus for other inflammatory cells as a producer of cytokines and as a source of antigenic material for lymphocyte activation. In order to fulfill their function, DCs must be able to take up antigenic material and migrate to local lymph nodes to present it to lymphocytes for proper activation and response. $\mathrm{PGE}_{2}$ has been shown to affect all aspects of DC function including activation, migration and co-stimulation of lymphocytes. The activation of DCs is augmented by $\mathrm{PGE}_{2}$ via the EP receptors. The secretion of the cytokines IL-12, CCL3, CCL4, tumor necrosis factor and IL-1 is abated in the presence of $\mathrm{PGE}_{2}$ during DC activation [162-165]. Under these same circumstances, DCs showed enhanced expression of chemokine receptors, such as CCR7 necessary for migration to lymph nodes [166]. The presence of $\mathrm{PGE}_{2}$ during $\mathrm{DC}$ maturation is essential for the acquirement of a migratory phenotype, as demonstrated by a lack of migration when $\mathrm{PGE}_{2}$ is not present from the onset of maturation [166]. Early in vivo studies using a transgenic mouse lacking the EP4 receptor demonstrated deficient recruitment of Langerhans cells [167]. In addition, the inhibition of EP2 and EP4 receptor signaling by antagonists demonstrated similar deficiencies in migration [167]. Consequently, agonists for EP2 or EP4 receptors are able to trigger DC migration, with both receptors being equally apt at facilitating migration $[162,166]$. Upon migrating to the lymph node, DCs are responsible for activating 
naïve and memory $\mathrm{T}$ cells, and therefore initiate the adaptive immune response. Studies using monocytes derived DCs (MoDCs) isolated from human blood demonstrated that $\mathrm{PGE}_{2}$ matured MoDCs have an enhanced potential to induced antigen-specific $\mathrm{T}$ cell proliferation. The co stimulatory molecules $\mathrm{OX} 40 \mathrm{~L}$ and $\mathrm{CD} 70$ expression on MoDCs is dependent on $\mathrm{PGE}_{2}$ stimulation [168]. These co stimulatory molecules induce proliferation of antigen-specific $\mathrm{T}$ cells when expressed by activated DCs during the adaptive immune response. Therefore, $\mathrm{PGE}_{2}$ enhances the proliferation of antigen specific $\mathrm{T}$ cells during the adaptive immune response by up regulation the co stimulatory molecules OX40L and CD70 [168].

$\mathrm{PGE}_{2}$ has been shown to regulate the proliferation, apoptosis and cytokine secretion of Tcells [169]. Studies have mainly focused on $\mathrm{CD}^{+} \mathrm{T}$-cells and have implicated that $\mathrm{PGE}_{2}$ has varied effects on their function and activation. The inhibition of T-cell proliferation by $\mathrm{PGE}_{2}$ is well established, but the mechanism of inhibition is still up for debate. $\mathrm{PGE}_{2}$ has been found to inhibit intracellular calcium release and the activity of p59 (fyn) protein tyrosine kinase; both could be the reason for decreased proliferation. EP receptors are also capable of inhibiting T-cell proliferation [170]. Studies have demonstrated that up regulation of certain EP receptors, $\mathrm{EP}_{4}$, induce a decrease in the secretion of IL-2 and therefore suppress T-cell proliferation [171].

$\mathrm{PGE}_{2}$ not only controls the proliferation of T-cells, it also regulates their survival, directly controlling the number and population of $\mathrm{T}$ cells present. $\mathrm{PGE}_{2}$ 's modulation of apoptosis is dependent upon the age and activation of the $\mathrm{T}$ cells. Naïve $\mathrm{CD} 4^{+} \mathrm{CD} 8^{+}$double positive thymocytes undergo apoptosis in vitro and in vivo when in the presence of $\mathrm{PGE}_{2}$ [172], as do resting mature T-cells by increased expression of the protein c-myc [173]. On the contrary, $\mathrm{PGE}_{2}$ is able to prevent $\mathrm{T}$ cell receptor (TCR)-mediated activation-induced cell death by augmenting Fas ligand (FasL) expression on the T-cell surface[174]. Inhibition of $\mathrm{T}$ cell 
apoptosis by $\mathrm{PGE}_{2}$ is also mediated by decreased FasL mRNA and protein expression [174]. Therefore, $\mathrm{PGE}_{2}$ is selectively induces apoptosis in immature thymocytes, allowing it to play a role in the shaping of the $\mathrm{T}$-cell repertoire. Furthermore, $\mathrm{PGE}_{2}$ regulates the activities of mature resting and activated $\mathrm{T}$ cells respectively, by inducing and inhibiting apoptosis,.

The influence of $\mathrm{PGE}_{2}$ on the adaptive immune response begins at the point of initiation with its ability to regulate dendritic cells whose activity controls the $\mathrm{T}$ cell adaptive immune response. The adaptive response can be type 1 or type 2 and is determined by the type of antigen present. The three effector $T_{h}$ subsets involved are $T_{h} 1, T_{h} 2$ and $T$ helper 17 cells $\left(T_{h} 17\right)$ with specific cytokines inducing the differentiation of each. $T_{h} 1$ cell differentiation is induced by IL$12, \mathrm{~T}_{\mathrm{h}} 2$ cell differentiation requires IL- 4 and $\mathrm{T}_{\mathrm{h}} 17$ differentiation is induced by TGF- $\beta$ and IL- 6 and is expanded by IL-23. $\mathrm{PGE}_{2}$ modulates both $\mathrm{DC}$ and $\mathrm{T}$ cell cytokine secretion that is involved in $\mathrm{T}$ helper cell differentiation [168], indicating $\mathrm{PGE}_{2}$ as a plausible regulator of the $\mathrm{T}$ cell phenotype in the adaptive immune response. Studies have shown that $\mathrm{PGE}_{2}$ decreases IFN$\gamma$ and IL-2 secretion by T cells, as well as DC secretion of IL-12, TNF and IL-1 [169]. However, $\mathrm{PGE}_{2}$ facilitates the expression of IL-23 by activated DCs [175]. Notably, IL-12 and IL-23 share the common p40 subunit as well as being responsible for $\mathrm{T}_{\mathrm{h}}$ cell differentiation. Combined, the ability of $\mathrm{PGE}_{2}$ to abate IL-12 production, while enhancing IL-23 production by DCs, directs the adaptive immune response towards a $\mathrm{T}_{\mathrm{h}} 17$ cell phenotype [176].

\section{$\mathrm{PGD}_{2}$ and Its Receptors}

$\mathrm{PGD}_{2}$ is the major PG produced by the COX pathway in activated mast cells which initiates IgE-mediated Type I acute allergic response [177-179]. It is released into the airway following antigen challenge by its major cellular source, mast cells, but is also produced by inflammatory cells, such as antigen presenting dendritic cells and $\mathrm{T}_{\mathrm{h}} 2$ cells, as well as 
fibroblasts, bronchial smooth muscle cells and airway epithelial cells [180-181]. Secretion of $\mathrm{PGD}_{2}$ elicits key components of allergic asthma such as bronchoconstriction and airway eosinophil infiltration by inducing vasodilatation and increased permeability in order to facilitate transendothelial migration of inflammatory cells [182-183]. Other function of this lipid mediator includes regulating the activation and chemotaxis of eosinophils, basophils and $T_{h} 2$ cells [149$150,184-185]$.

In order to exert its biological effects, $\mathrm{PGD}_{2}$ must bind and activate two distinct GPCRs, the D prostanoid (DP) receptor and the recently discovered CRTH2 receptor ("DP2"). The DP receptor is a member of prostanoid GPCR super family and signals to $\mathrm{G}_{\mathrm{s}}$-mediated increases in intracellular cAMP. This promotes relaxation of both vascular and airway smooth muscle, inducing vasodilation and vascular permeability, promoting extravasation and infiltration of inflammatory cells. Despite avid interest there has not been an established connection between DP receptor activation and $\mathrm{PGD}_{2}$-stimulated eosinophil activation and migration [186-187]. The identification of the novel receptor, $\mathrm{CRTH} 2$, has provided clarification of the mechanism behind $\mathrm{PGD}_{2}$ biological activity $[149,188] . \quad \mathrm{CRTH} 2$ is considered an orphan receptor that is predominantly expressed on type 2 polarized lymphocytes [149]. Though DP and CRTH2 receptors exhibit equivalent binding affinity for $\mathrm{PGD}_{2}$, they share no similarities and are not structurally related. Rather, CRTH2 is most closely related to other chemoattractant receptors. The effects of CRTH2 are mediated through $\mathrm{G}_{\mathrm{i}}$-dependent increases in intracellular calcium levels and reduction in intracellular cAMP levels, indicative of proinflammatory signaling [149, 189]. These pathways have been shown to mediate eosinophil activation by regulating the cells shape change, actin polymerization and $\mathrm{CD} 11 \mathrm{~b}$ up regulation $[150,190]$. Therefore, the 
combination of these two receptors help to create an inflammatory environment that contributes to allergic disease.

The pro-inflammatory effects of $\mathrm{PGD}_{2}$ are mediated by both receptors via multiple signaling pathways whose activity is dependent on local receptor expression. DP receptors are predominantly expressed on bronchial epithelium indicating that receptor activation mediates production of cytokines and lipid mediators that recruit DP receptor expressing cells such as: platelets, fibroblasts, DCs, T cells, basophils, eosinophils and mast cells [191]. Currently there are several experimental models that support and demonstrate DP receptor mediated airway inflammation and hyper-reactivity. DP antagonists promote anti-inflammatory properties in experimental models that include the inhibition of antigen-induced conjunctivial micro vascular permeability in guinea pigs [192] and attenuated ovalbumin (OVA)-induced hyper-reactivity in mice [193]. The pro-inflammatory effect of DP signaling is further exemplified by the observation that mice deficient in the DP receptor have diminished airway hyper-sensitivity [194]. Therefore, DP receptor signaling is key in mediating the effects of mast cells released $\mathrm{PGD}_{2}$ on the airway epithelium inducing the release of specific cytokines and chemokines, such as IL-8 and GM-CSF, creating airway inflammation, obstruction and hyper-reactivity [194].

$\mathrm{PGD}_{2}$ released by mast cells also mediates the activation, chemotaxis and infiltration of inflammatory cells that express the $\mathrm{CRTH} 2$ receptor. This high affinity $\mathrm{PGD}_{2}$ receptor is expressed in type 2 polarized lymphocytes [195], basophils, eosinophils [196] and monocytes [197]. Signaling via CRTH2 is responsible for the activation and recruitment of $\mathrm{Th}_{2}$ cells, eosinophils, basophils and monocytes, initiating and driving eosinophilic and inflammatory cell infiltration $[149,197]$. The chemotactic response to $\mathrm{PGD}_{2}$ by eosinophils, basophils and $\mathrm{Th}_{2}$ cells is mediated by CRTH2. This is substantiated by in vitro and in vivo studies that confirm 
the chemotaxis of these cell types is in response to $\mathrm{PGD}_{2}$ signaling through the CRTH2 receptor not DP. Specifically the, antagonism of CRTH2 receptor exhibited greatly decreased eosinophilic infiltration post antigen exposure in ramatroban-treated animals [198-199]. This was further validated by studies of a $\mathrm{PDG}_{2}$ double knockout model by Gervais et al.[200]. Treatment with a CRTH2 agonist stimulated chemokine release and eosinophil degranulation, where as treatment with the DP agonist BW245C did not elicit these effects. In this same study CRTH2 activation also lead to eosinophil shape changes, chemotaxis, enhancement of chemotactic responsiveness to other chemoattractants and degranulation, where as stimulation through DP activation induced eosinophil apoptosis. Blocking DP activation using a selective antagonist BW A868C significantly enhances CRTH2-mediated activation indicated by increased $\mathrm{CD} 11 \mathrm{~b}$ expression post $\mathrm{PGD}_{2}$ stimulation [149-150]. Therefore, $\mathrm{PGD}_{2}$ activation and recruitment of eosinophils is controlled by a dual receptor system that requires collaboration between the two high affinity receptors, DP and CRTH2.

$\mathrm{PGD}_{2}$ can act not only as a pro-inflammatory mediator during allergic inflammation, but also has the ability to act as an inhibitor of inflammation in specific contexts via DP receptor signaling. The DP receptor is expressed by DCs that are responsible for initiating adaptive immune response when they come into contact with possible antigens. Studies done by Hammad et al. [201] using a FITC-OVA mouse model of inflammation to observe dendritic cell activation and migration. They show that the intratracheal instillation of FITC-OVA together with $\mathrm{PGD}_{2}$ inhibits the migration of FITC $^{+}$lung DC to draining LNs [201]. Decreased DC migration decreases antigen specific $\mathrm{T}$ cell activation, proliferation and the production of antigen specific cytokines. Under steady state conditions, macrophages might secrete $\mathrm{PGD}_{2}$ to suppress $\mathrm{DC}$ and $\mathrm{T}$ cell activation [202]. During inflammation, $\mathrm{PGE}_{2}$ and $\mathrm{LTC}_{4}$ are produced and induce 
differentiation and maturation of tissue-resident DCs [167, 203]. Under these conditions, the anti-inflammatory effects of $\mathrm{PGD}_{2}$ are overridden by the pro-inflammatory effects of $\mathrm{PGE}_{2}$, $\mathrm{LTC}_{4}$, and pro-inflammatory cytokines. Also, at the resolution of inflammation, COX-2 enzyme mainly generates $\mathrm{PGD}_{2}$, rather than $\mathrm{PGE}_{2}$ [204]. The release of $\mathrm{PGD}_{2}$ might suppress $\mathrm{DC}$ migration to prevent further immune stimulation, thus contributing to the resolution of inflammation.

The biological activity of $\mathrm{PGD}_{2}$ signaling is not restricted to the inflammatory effects of asthma alone, but is pertinent in the progression from chronic unresolved inflammation into permanent remodeling of the airway. When airway inflammation is not adequately treated, it can result in permanent structural changes in the airways. such as increased airway wall thickness that involves both smooth muscle and collagen tissue and increased mucous production due to proliferation of airway epithelial goblet cells resulting in mucus hypersecretion. Antigen induced expression of the high affinity DP receptor has been observed in both mouse bronchiole epithelial cells and human nasal epithelial goblet cells. DP receptor expression combined with the ability of DP signaling to induce mucus secretion by colonic goblet cells insinuates the possibility that DP receptors may provoke mucus secretion in response to $\mathrm{PGD}_{2}$ in asthma and allergic rhinitis.

\section{PGs and Airway Remodeling}

PGs play an important role in maintaining airway homeostasis, by inhibiting remodeling processes. This is best exemplified under the normal condition of the airway. Under normal circumstances, the epithelium releases mediators, specifically $\mathrm{PGE}_{2}$, that restrict mesenchymal cell proliferation and activation [205]. Upon injury to the epithelium $\mathrm{PGE}_{2}$ production is diminished resulting in the induction of repair responses that promote remodeling. 
Subsequently, the various mediators and growth factors that support the proliferation and survival of mesenchymal cells are released. Specifically, fibroblast growth factor (FGF), insulin growth factor (IGF), platelet-derived growth factor (PDGF), endothelin-1 (ET-1) and TGF- $\beta$ [206]. These factors induce inflammation and activation of cells involved in the repair response, specifically fibroblasts. Therefore, $\mathrm{PGE}_{2}$ suppresses remodeling as demonstrated by the maintenance of homeostasis when it is present in the airway under normal conditions.

Fibroblasts are the critical cells type involved in normal wound healing and whose apoptosis is required to prevent excessive matrix deposition or scarring. They also produce numerous mediators that induce proliferation and activation of remodeling processes. A critical feature of fibrotic lung disease is increased transition of fibroblasts into myofibroblasts or fibroblast like cells expressing $\alpha$-SMA [207]. Furthermore, in areas of active fibrosis there are increased numbers of myofibroblasts that are associated with promoting fibrotic processes $[50$, 208]. Myofibroblasts are the predominant source of increased collagen gene expression and production as demonstrated by the correlation between decreased lung compliance and their presence [209]. Not only do myofibroblasts alter tissue contractility and increase ECM synthesis, they also secrete mediators of fibrogenesis. Myofibroblasts secrete two specific substances: monocyte chemotractant protein-1 (MCP-1) and TGF- $\beta 1$ that induce inflammatory and fibrogenic processes [210-211]. These characteristics of myofibroblasts establish this cells type as a key catalytic factor in fibrosis [212]. $\mathrm{PGE}_{2}$ is a potent mediator of fibroblast activity, inhibiting their proliferation [213-214], survival [215], differentiation [216], growth factor expression [217], migration [218] and collagen synthesis [219-220]. By augmenting fibroblast/myofibroblast activity, $\mathrm{PGE}_{2}$ can regulate the fibrotic processes involved in the 
development of disease and could be a relevant factor involved in avoiding pathological processes.

Regulation of fibroblast proliferation involves two sides: an inducer of proliferation and a suppressor of proliferation, that act in opposition to maintain homeostasis. Induction of proliferation occurs during injury and inflammation due to both decreased suppression and increased pro-proliferative signals. For example, during inflammation AMs play a key role in initiating fibroblast proliferation by secreting fibronectin and macrophage-derived growth factor (AMDGF) [213]. In situations of injury and inflammation numerous secreted mediators such as TGF- $\beta$, FGF and IGF-1 increase due to infiltrating inflammatory cells or injured epithelium. At the conclusion of inflammation or when repair has occurred suppression of profibrogenic mediators returns and the processes of repair cease. Recently, it has been suggested that alveolar epithelial cells (AECs) are the primary producers of suppressive signals and that $\mathrm{PGE}_{2}$ is the key mediator. This is supported by early studies by Bitterman et al. [213] that determined that $\mathrm{PGE}_{2}$ inhibits fibroblast proliferative response to fibronectin and AMDGF in a dose dependent manner. This demonstrated for the first time that $\mathrm{PGE}_{2}$ is an inhibitor of fibroblast proliferation. Further studies determined that $\mathrm{PGE}_{2}$ is a relevant endogenous anti-proliferative mediator of fibroblasts because mice deficient in $\mathrm{PGE}_{2}$ demonstrate exaggerated fibroblast proliferation [221-222]. Therefore, it was determined that AECs suppressed fibroblast proliferation by secreting $\mathrm{PGE}_{2}$ and loss of AEC suppression results in repair. The importance of $\mathrm{PGE}_{2}$ suppression of profibrogenic processes is key in avoiding fibroproliferative diseases and may be relevant in understanding the processes involved.

Fibroblast differentiation into myofibroblasts is necessary for wound healing or remodeling to occur [223]. The main function of myofibroblasts is to form adhesions between 
the wound edge and the ECM. These adhesions allow them to pull the ECM when they contract, reducing the wound size by pulling the wound edges together. Fibroblasts lay down collagen to reinforce the wound as myofibroblasts contract to allow for complete repair [40]. $\alpha$-SMA provides the contractile ability to fibrocytes and is the main identifying marker of myofibroblasts [51, 208-209]. At least three local events are needed to generate $\alpha$-SMA-positive differentiated myofibroblasts: 1) accumulation of biologically active TGF- $\beta 1,2)$ the presence of specialized ECM proteins like the ED-A splice variant of fibronectin, and 3) high extracellular stress, arising from the mechanical properties of the ECM and cell remodeling activity $[40,208]$. IL-1 $\beta$ and IFN- $\gamma$ have been shown to down regulate the expression of $\alpha$-SMA mRNA and protein as well as decrease fibroblast proliferation and collagen synthesis [209, 224-225] . More importantly, $\mathrm{PGE}_{2}$ was shown by Kolodsick et al. [216] to inhibit TGF- $\beta 1$ induced $\alpha$-SMA expression through the $\mathrm{EP}_{2}$ receptor. $\mathrm{PGE}_{2}$ is able to limit synthesis of matrix collagen and prevent fibroblast transition, two prominent components of remodeling [216]. It also induces fibroblast apoptosis, preventing excessive matrix deposition or remodeling [215]. Huang et al. [215] demonstrated that $\mathrm{PGE}_{2}$ signaling via EP2 or EP4 induced fibroblast apoptosis. Apoptosis was modulated by different survival pathways such as activation of both caspase 8 and 9 [215], Fas ligand [215] and enhanced phosphatase and tensin homolog (PTEN) activity [218, 226]. Signaling through EP2 also prevents fibroblast chemotaxis and migration as well as its production of collagen [218-219]. Therefore, $\mathrm{PGE}_{2}$ is a key regulator of fibroblast activity [227].

$\mathrm{PGE}_{2}$ not only regulates the fibroproliferative aspects of remodeling, it also suppresses the inflammatory component. Inflammation is a key component in remodeling, where the inflammatory response provides the cells and mediators responsible for repair. $\mathrm{PGE}_{2}$ opposes leukocyte accumulation through numerous different mechanisms. It inhibits myelopoiesis, 
diminishing the available cells who could participate. It also inhibits the recruitment and chemotaxis of inflammatory cells deterring their accumulation. The activation of cells and secretion of inflammatory mediators that are involved in remodeling is also effected by $\mathrm{PGE}_{2}$. $\mathrm{PGE}_{2}$ negates the production of a variety of inflammatory mediators, including reactive oxygen species, IL-8, TNF- $\alpha$, FGF, ET-1 and LTs. It also up-regulates the expression of the suppressive mediator IL-10, which also induces anti-inflammatory aspects. This demonstrates that $\mathrm{PGE}_{2}$ is paramount in suppressing aspects of the inflammatory response that could lead to remodeling.

\section{Leukotrienes (LTs) Synthesis}

The synthesis of LTs is initiated by 5-lipoxygenas (5-LO) with the assistance of 5lipoxygenase activating protein (FLAP) [228] as shown in Figure 7. FLAP serves as a membrane anchor for 5-LO and acts as an amine bind protein to enhance its ability to interact with its substrate [229]. More importantly it is necessary for the activation of 5-LO's enzymatic activity and therefore essential for LT synthesis [230-231]. FLAP and 5-LO oxygenate arachidonic acid to produce leukotriene $\mathrm{A}_{4}\left(\mathrm{LTA}_{4}\right)$, the unstable precursor of all LTs [232]. LTA $_{4}$ is either conjugated with reduced glutathione by leukotriene $\mathrm{C}_{4}$ synthase making leukotriene $\mathrm{C}_{4}\left(\mathrm{LTC}_{4}\right)$ or hydrolyzed by leukotriene $\mathrm{A}_{4}$ hydrolase to form leukotriene $\mathrm{B}_{4}\left(\mathrm{LTB}_{4}\right)$ [233]. Both $\mathrm{LTB}_{4}$ and $\mathrm{LTC}_{4}$ are then transported out of the source cell by specific transporter proteins [233]. Exported $\mathrm{LTC}_{4}$ undergoes sequential peptide cleavage events to produce leukotriene $\mathrm{D}_{4}\left(\mathrm{LTD}_{4}\right)$ and finally leukotriene $\mathrm{E}_{4}\left(\mathrm{LTE}_{4}\right) . \mathrm{LTC}_{4}, \mathrm{LTD}_{4}$ and $\mathrm{LTE}_{4}$ are known as the cysteinyl leukotrienes (cysLTs).

Leukocytes are the primary producers of LTs because they are the only cells that express sufficient 5-LO and FLAP to synthesize appreciable amounts [138]. Incidentally, non leukocyte cells that express distal $\mathrm{LTA}_{4}$ metabolizing enzymes can take up leukocyte derived $\mathrm{LTA}_{4}$ and 
produce LTs by a process called transcellular biosynthesis [234]. The primary example of this process is the interaction between neutrophils and endothelial cells. In this case, neutrophils produce and release $\mathrm{LTA}_{4}$ that endothelial cells take up and metabolize into $\mathrm{LTC}_{4}$ using their $\mathrm{LTC}_{4}$ synthase, demonstrating another source of LTs.

\section{LT Receptors}

LTs exert their biological activity by binding to specific heptahelical receptors of the rhodopsin class that are expressed on the outer plasma membrane of structural and inflammatory cells [235-236]. These receptors signal through $G$ proteins in the cytoplasm that induce increases in intracellular calcium and reductions in intracellular cAMP. Changes in intracellular cAMP and $\mathrm{Ca}^{2+}$ activates different downstream kinase cascades that can then alter cellular activity [233]. Resulting changes in cellular activity is dependent upon the LT and cell types involved as shown in figure 10 . 


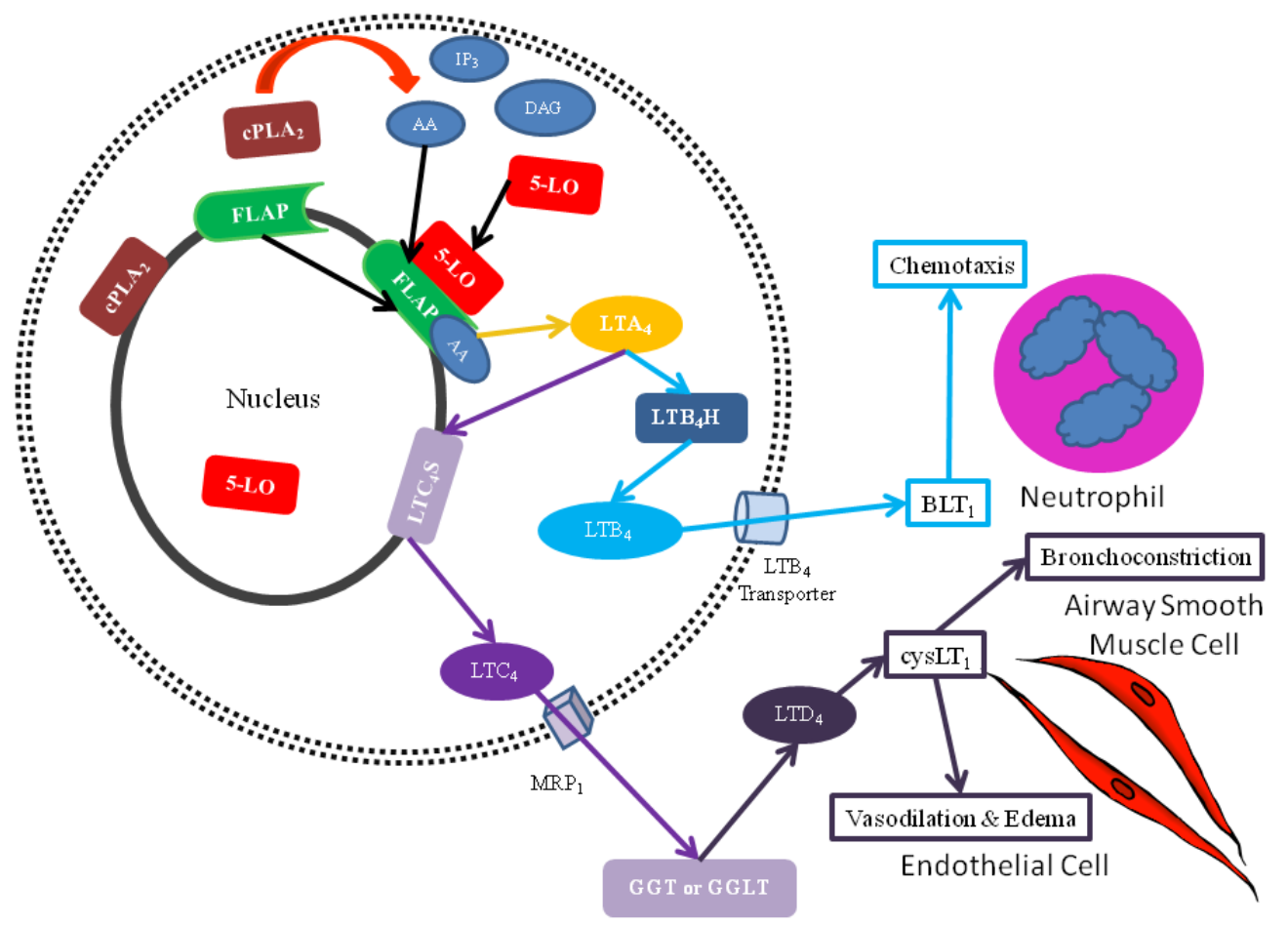

Figure 10 LT Activity

Leukotrienes synthesis, secretion, receptor binding and biological action. $\mathrm{LTA}_{4}$ and $\mathrm{LTB}_{4}$ can potentially be formed on either side of the nuclear envelope by nuclear- or cytosolic-localized pools of 5-LO and LTA 4 hydrolase in macrophages and mast cells. The $\mathrm{LTB}_{4}$ transporter can facilitate transfer of $\mathrm{LTB}_{4}$ out of the cell, where it can act on neutrophils through the BLT $\mathrm{B}_{1}$ receptor, inducing chemotaxis and recruitment. LTA 4 can also be converted to $\mathrm{LTC}_{4}$ by $\mathrm{LTC}_{4}$ synthase, a FLAP-like protein found in the nuclear envelope. The multidrug resistance-associated protein-1 (MRP1) can transport $\mathrm{LTC}_{4}$ out of the cell, where it is metabolized by extracellular-localized g-glutamyl transpeptidase (GGT) or g-glutamyl leukotrienase (GGLT) to LTD . LTD $_{4}$ can bind to the cysLT $T_{1}$ receptor on both endothelial cells and airway smooth muscle cells or be further metabolized to make $\mathrm{LTE}_{4}$.

LT receptors were originally divided into two classes known as B leukotriene receptors (BLT) and CysLT receptors based on ligand preference. There are two BLT receptors, BLT $_{1}$ and $\mathrm{BLT}_{2}$ that preferentially bind $\mathrm{LTB}_{4}$ (Figure 8). BLT 1 is the high affinity receptor for $\mathrm{LTB}_{4}$ that mediates almost all of its signaling, whereas the role of the lower affinity $\mathrm{BLT}_{2}$ receptor is not well known. There are two cysLT receptors: type 1 and type 2 cysteinyl leukotriene receptor $\left(\operatorname{cysLT}_{1}\right.$ and $\left.\operatorname{cysLT}_{2}\right) . \mathrm{CysLT}_{1}$, mediates sustained bronchoconstriction, mucus secretion and edema in the airways [237]. CysLT $_{2}$ is responsible for mediating inflammation, vascular 
permeability and tissue fibrosis [237]. Combined these receptors induce the different biological effects of $\mathrm{LTA}_{4}$ and the cysLTs.

\section{LTs and Airway Inflammation}

\section{$\mathrm{LTB}_{4}$}

$\mathrm{LTB}_{4}$ is a potent lipid inflammatory mediator involved in regulating aspects of allergic airway inflammation and the pathogenesis of asthma. $\mathrm{LTB}_{4}$ is a prominent chemoattractant for PMNs [238], but it also influences the chemotaxis of monocytes, macrophages and eosinophils [239-240]. In addition to its potency as a chemoattractant, $\mathrm{LTB}_{4}$ serves as an activator of PMNs through $\mathrm{BLT}_{1}$ receptor signaling [241]. PMN BLT 1 receptor signaling promotes two functions that are key components of PMN inflammatory responses. BLT 1 receptor ligation encourages cPLA2 cleavage of membrane phospholipids releasing arachidonic acid that is converted into LTs [242]. Also, $\mathrm{LTB}_{4}$ signaling induces PMN degranulation resulting in release of inflammatory mediators that can dictate the local inflammatory response [243]. Therefore, $\mathrm{LTB}_{4}$ is an important mediator of the early phases of inflammation that include the recruitment and activation of inflammatory cells that create the inflammatory environment.

MCs play a central role in inflammatory and immediate allergic reactions. Studies by Weller et al. [244] revealed that $\mathrm{LTB}_{4}$ acts as an autocrine mediator of MCs. In this study, MC progenitor cells derived from either BALB/C bone marrow or heparin treated human umbilical cord blood were shown to express the $\mathrm{BLT}_{1}$ receptor, indicating $\mathrm{LTB}_{4}$ involvement in early mast cell activation. Result of an in vitro chemotaxis assay demonstrated that $\mathrm{LTB}_{4}$ released by activated MC is an effective chemotractant for MC progenitors. In addition, in vivo studies demonstrated the accumulation of adoptively transferred fluorescent labeled c-Kit ${ }^{+} \mathrm{MC}$ progenitor to the site of subcutaneous injection of $\mathrm{LTB}_{4}$. Weller et al.'s results indicate that 
$\mathrm{LTB}_{4}$ is a potent chemoattractant for MC progenitor cells, but not for mature MCs. The response to $\mathrm{LTB}_{4}$ is temporal, dissipating with the maturing of MCs which corresponds with the decrease in $\mathrm{BLT}_{1}$ receptor mRNA expression and chemotaxis by mature MCs. This temporal shift of $\mathrm{LTB}_{4}$ sensitivity allows for immature MCs to be recruited to sites of inflammation and prevents concentration of mature MCs to the site of focal allergen stimulus, depleting mature MCs from the periphery.

It was more recently determined that $\mathrm{LTB}_{4}$ signaling through $\mathrm{BLT}_{1}$ is an important mediator of $\mathrm{T}$ cell recruitment and activation in allergic inflammation [245]. Miyahara et al. [246] found that during the course of naive $\mathrm{CD}^{+} \mathrm{T}$ cell differentiation, the $\mathrm{LTB}_{4}$ receptor $\mathrm{BLT}_{1}$ is expressed and functional on $\mathrm{T}_{\mathrm{h}} 1$ and $\mathrm{T}_{\mathrm{h}} 2$ effector cells. In addition, antigen-specific effector $\mathrm{CD}^{+} \mathrm{T}$ cells generated in vivo up-regulated $\mathrm{BLT}_{1}$ when they migrated out of the lymphoid compartment and were recruited into tissue. BLT $\mathrm{T}_{1}$ mediated $\mathrm{LTB}_{4}$-induced $\mathrm{T}_{\mathrm{h}} 1$ and $\mathrm{T}_{\mathrm{h}} 2$ cell chemotaxis and firm adhesion to endothelial cells under flow. Furthermore, $\mathrm{BLT}_{1}$ mediated early $\mathrm{CD}^{+}$and $\mathrm{CD}^{+} \mathrm{T}$ cell recruitment into the airway in a model of allergic pulmonary inflammation. This indicates that the $\mathrm{LTB}_{4}-\mathrm{BLT}_{1}$ pathway is involved in linking early immune system activation and early effector $\mathrm{T}$ cell recruitment.

In conjunction with $\mathrm{LTB}_{4}$ and $\mathrm{BLT}_{1}$ ability to recruit effector $\mathrm{T}$ cells, it can also play an important role in initiating and developing a $\mathrm{T}_{\mathrm{h}} 2$-type immune response that is focal in airway hyper responsiveness (AHR) $[245,247]$. In the absence of BLT $_{1}$ expression, development of AHR was attenuated, but not completely abrogated in mice in an OVA-induced bronchial asthma model [246]. $\mathrm{BLT}_{1}^{-/}$mice developed significantly lower airway responsiveness to inhaled methacholine, lower goblet cell hyperplasia, decreased IgE expression and decreased $T_{h} 2$ cytokine expression, specifically IL-13 production in vivo and in vitro as well as IL-5 in vivo 
[246]. It was also determined that the development of $\mathrm{T}_{\mathrm{h}} 2$ cells was also impaired in the absence of $\mathrm{BLT}_{1}$ [247]. When $\mathrm{BLT}_{1}{ }^{-/-}$mice received allergen sensitized $\mathrm{BLT}_{1}^{+/+} \mathrm{T}$ cells through adoptive transfer the development of AHR was fully restored as well as $T_{h} 2$ cytokine production and $T_{h} 2$ cell development [246]. Thus $\mathrm{LTB}_{4}$ and $\mathrm{BLT}_{1}$ interaction plays an important role in initiating and developing $\mathrm{T}_{\mathrm{h}} 2$ type immune response and the full development of AHR.

\section{Cysteinyl Leukotrienes (cysLTs)}

CysLTs are recognized as potent inflammatory mediators that initiate and propagate a diverse array of biological responses in the airway. They are best known for their contractile effects on the smooth muscle and endothelium [248-249]. For example, in the airway cysLTs bind to cysLT $T_{1}$ receptors expressed on airway smooth muscle resulting in bronchoconstriction [237]. CysLT regulation of muscle tone is intimately associated with the regulation of vascular permeability and vasoconstriction [249]. Changes in vascular permeability are key in allowing for inflammatory cell extravasation and migration to different sites of inflammation. Changes in permeability also control plasma protein leakage which can develop into edema. Therefore, cysLTs signaling through cysL $\mathrm{T}_{1}$ plays an important role in the muscle tone of the airway and vasculature.

CysLTs serve as a chemotactic mediator and activation stimulus for effector leukocytes such as macrophages, monocytes and eosinophils. CysLTs have been shown to influence both progenitor and differentiated immune cells through both receptors. It was observed that the cysLT $\mathrm{L}_{1}$ receptor, but not cysLT $\mathrm{T}_{2}$ receptor, are expressed on $\mathrm{CD}^{+} 4^{+}$peripheral blood derived progenitor cells [250]. In vitro treatment of these cells with cysLTs induced transendothelial migration, which was inhibited by pretreatment with Monteleukast (cysLT $\mathrm{T}_{1}$ receptor antagonist) [250]. Monteleukast inhibition was not present in mature peripheral blood leukocytes, indicating 
that the cysLT $T_{1}$ receptor is no longer the primary means of leukocyte signaling, with the cysLT receptor taking its place. $\mathrm{CysLT}_{2}$ receptor expression occurs during development and may be important in differentiating progenitor cell and mature cell cysLT signaling. CysLT signaling is a stimulus for activation of peripheral leukocytes and is dependent upon the expression of cysLT receptors which is regulated by cytokines. For example, treatment of peripheral blood monocytes or macrophages with IL-4 or IL-13 induces increased cysLT ${ }_{1}$ receptor expression [251]. Enhanced receptor expression results in amplified cysLT chemotaxis.

CysLTs pro-inflammatory characteristics have long been associated with the activity of eosinophils in allergic inflammation. The migration of eosinophils into the airway involves several steps; including eosinophilopoesis and release from the bone marrow, up regulation of adhesion molecules on eosinophils as well as on the vascular endothelium and the increase in chemoattractant molecules released into the airways. CysLTs have been associated with each aspect of increased airway eosinophilia. The increase in the number of eosinophils through eosinophilopoesis is regulated by cysLT $T_{1}$ receptor signaling in combination with IL-13 and eotaxin [252]. In addition, the proliferation of eosinophils that occurs during allergic reactions is controlled by $\mathrm{LTD}_{4}$ in combination with the growth factors, granulocyte-macrophage colonystimulating factor (GM-CSF) and IL-5 [253]. Therefore, cysLT signaling increases the number of eosinophils present in the airway. Eosinophil recruitment is facilitated by cysLT regulation of adhesion molecules on both the endothelium and on eosinophils. P-selectin, an adhesion molecule that is expressed on activated endothelial cells, function to recruit eosinophils and other leukocytes to the site of inflammation. CysLTs $\mathrm{LTC}_{4}$ and $\mathrm{LTD}_{4}$ induce surface expression of endothelial P-selectin [254], and further assist in the accumulation of eosinophils. To increase eosinophil migration, cysLTs also up regulate $\beta_{2}$-integrin expression on eosinophils, stimulate 
vascular endothelial cells to produce PAF and increase adhesion of leukocytes [255-256]. The Combined efforts of cysLT signaling are meant to increase eosinophil chemotaxis to sites of inflammation. Once they arrive, cysLT signaling is able to induce survival of eosinophils in order to maintain the inflammatory reaction [257]. Therefore, cysLT activity is prominently involved in controlling eosinophil differentiation, proliferation, chemotaxis, migration and survival.

CysLTs influence the adaptive immune response through their ability to mediate the maturation and migration of DCs [203]. In vivo studies using multidrug-resistance associated protein-1 deficient mice, which have impaired responses to arachidonic acid stimulated inflammation because this protein is the major transporter for $\mathrm{LTC}_{4}$, exhibited significantly suppressed DC migration compared with wild type controls [203]. Injection of $\mathrm{LTC}_{4}$ or $\mathrm{LTD}_{4}$ reversed the suppression of DC migration [203]. DCs produce endogenous $\mathrm{LTC}_{4}$ when initially exposed to antigen which serves as a critical determinant for homing to regional lymph nodes, indicating why disruption of $\mathrm{LTC}_{4}$ synthesis suppressed DC migration.

DC migration is an important step in the activation of an adaptive immune response, but not the only important function of DCs in regulating the adaptive response. DCs also play an important part in controlling the type of $\mathrm{T}$ cell response based on the type of antigen and chemical mediators present. CysLTs play an important part in directing DC immunomodulatory functions. In order to determine the effect of cysLTs on airway inflammation Machida et al. [258] used murine bone marrow derived DCs pulsed with mite allergens, Dermatophagoides farinea (Der-f) and then analyzed for production of in vitro cytokines with or without exogenous cysLT treatment. Preliminary findings demonstrated that DCs produce cysLTs in response to aeroallergens and express the cysLT1 receptor. Analysis of cytokine production showed $\operatorname{Der} f$ 
induced increased production of IL-10 and IL-12 compared to controls. Der $f$ and cysLT pulsed DCs produced significantly higher levels of IL-10 compared to $\operatorname{Der} f$ alone but with similar IL12 levels. Treatment of DC with Der $f$ and the cysLT1 antagonist LTRA demonstrated increased levels of IL-12 and decreased levels of IL-10. This is important because higher levels of IL-12 and low levels of IL-10 are associated with the development of a $T_{h} 1$ response [259-262], while higher levels of IL-10 and lower levels of IL-12 are associated with a $T_{h} 2$ response [263]. Therefore, these results demonstrate that cysLTs induce the production of a cytokine profile promoting a $\mathrm{T}_{\mathrm{h}} 2$ response, with higher levels of IL-10 and lower levels of IL-12. The pulsed DCs were then subsequently instilled into the airways of naïve mice that were then exposed to Der $f$ to determine in vitro effects of cysLTs on DCs. They analyzed the pathology and BAL cytokine profiles of the mice to find that instillation with $\operatorname{Der} f$ DCs induced allergic airway inflammation characterized by increased eosinophilia and IL-5 production. The addition of the cysLT, $\mathrm{LTD}_{4}$, leads to an even greater number of eosinophils in the airway and increased production of IL-5. In contrast, mice instilled with DCs pulsed with $\operatorname{Der} f$ and LRTA showed reduced eosinophilia and IL-5 production in the airway, but demonstrated increased IFN- $\gamma$. Therefore, in vivo and in vitro studies demonstrated that cysLT treatment influences airway DCs to initiate a $\mathrm{T}_{\mathrm{h}} 2$ response, directly affecting the adaptive immune response.

CysLTs influence over the adaptive immune response not only involves the regulation of DC immunomodulatory functions, but also involves regulating the initiation and amplification of $\mathrm{T}_{\mathrm{h}} 2$ cell dependent inflammatory responses. $\mathrm{T}_{\mathrm{h}} 2$ cell dominant inflammatory responses are a prominent component of asthma and the development of AHR [264-265]. LTB $_{4}$ and BLT $_{1}$ signaling have been established as key factors in the regulation AHR mechanisms [247, 266]. These mediators are known as inducers of AHR by their ability to recruit and activate IL-13 
producing effector/memory $\mathrm{T}$ cells, but they also work in concert with cysLTs [267]. Kim et al. [268] used mice with a targeted disruption of $\mathrm{LTC}_{4}$ synthase to determine the role of cysLTs in the development of $\mathrm{T}_{\mathrm{h}} 2$ cell dependent inflammation in the airways. They found that $\mathrm{LTC}_{4} \mathrm{~S}^{\text {null }}$ mice diminished manifestations of $\mathrm{T}_{\mathrm{h}} 2$ cell dependent pulmonary inflammation. More specifically, accumulation of eosinophils and lymphocytes, goblet cell hyperplasia and mucus

production, IL-4, IL-5 and IL-13 transcription and secretion and serum IgE and IgG1 were all diminished in $\mathrm{LTC}_{4} \mathrm{~S}^{\text {null }}$ mice. This demonstrates that cysLTs are key in regulating the adaptive immune response in their ability to initiate or amplify $\mathrm{T}_{\mathrm{h}} 2$ dependent inflammation.

\section{Leukotrienes and Airway Remodeling}

The key features of airway remodeling include: the accumulation and activation of inflammatory cells, a $\mathrm{T}_{\mathrm{h}} 2$ cell immune response phenotype, mesenchymal cell hyperplasia and increased matrix protein deposition. Looking at theses hallmarks of airway remodeling it is apparent that LTs play an important role in the process. LTs increase the number of inflammatory cells present in the airway through various modes of action. LTB $_{4}$, as stated above, is known to be a potent chemoattractant for neutrophils, eosinophils, macrophages and lymphocytes (effector $\mathrm{T}$ cells). CysLTs provide increased means of inflammatory cell extravasation by up regulating endothelial cell adhesion molecule expression. Additionally, LT signaling can inhibit apoptosis of recruited cells, such as neutrophils and eosinophils. When the activity of LTs is blocked either through inhibition of synthesis or receptor antagonism inflammatory cell accumulation is attenuated. Therefore it is apparent that LTs play an important role in the first part of airway remodeling.

$\mathrm{T}_{\mathrm{h}} 2$ cell dominated inflammatory responses are associated with the development of AHR and remodeling. cysLTs and $\mathrm{LTB}_{4}$ have been shown to regulate the initiation and amplification 
of a $\mathrm{T}_{\mathrm{h}} 2$ dominant immune response. $\mathrm{LTB}_{4} \mathrm{BLT} 1$ interaction is mandatory for the recruitment of $\mathrm{T}$ cells and the initiation and development of a $\mathrm{T}_{\mathrm{h}} 2$-type immune response [246] as exemplified by reduced AHR associated with decreased IL-5, IL-4 and IL-13 production in $\mathrm{BLT}^{\text {null }}$ mice. Specifically, $\mathrm{LTB}_{4}-$ BLT1 roles include: influencing DC migration to lymph nodes for T cell activation [246], attracting and activating lymphocytes (CD4 and CD8 T cells) [266], recruiting and activating IL-13 producing T cells [269] and stimulating T cell production of IL-4 and IL-5 [247]. Similarly, cysLTs regulate the initiation and amplification of $\mathrm{T}_{\mathrm{h}} 2$ celldependent inflammation. They influence airway DCs to initiate a $T_{h} 2$ response [258] and induce the production of $\mathrm{T}_{\mathrm{h}} 2$ cytokines IL-4 and IL-5 [268]. Combined, the cysLTs and $\mathrm{LTB}_{4}$ are prominent mediators involved in the initiation, activation and development of $\mathrm{T}_{\mathrm{h}} 2$ immune responses and are therefore relevant to the process of airway remodeling.

Mesenchymal cell hyperplasia results in airway smooth muscle cell and fibroblast proliferation. Studies have implicated that LTs augment both cell types and are key in inducing proliferation. Studies by Vannella et al [270] demonstrated that cysLTs regulate fibrocyte function via autocrine and paracrine signaling. They found that $\mathrm{LTD}_{4}$ induces fibrocyte proliferation to greater extent than $\mathrm{LTC}_{4}[270]$. The mitogenic properties of $\mathrm{LTD}_{4}$ are solely mediated by the cysL $\mathrm{T}_{1}$ receptor as demonstrated by augmented fibrocyte proliferative response to $\mathrm{LTD}_{4}$ when treated with cytLT 1 antagonists [271]. $\mathrm{LTD}_{4}$ along with $\mathrm{LTB}_{4}$ has also been shown to augment airway smooth muscle cell (ASM) proliferation when combined with epidermal growth factor (EGF) or IGF [272]. However, LT signaling combined with growth factors has no implication in altering collagen or ECM proteins.

CysLTs may modulate extracellular matrix remodeling during pulmonary inflammation. Primarily, cysLTs are able to influence fibroblast activity, specifically they induce the secretion 
of FGF which can induce increased collagen synthesis. Studies have implicated that collagen synthesis can be induced by $\mathrm{LTC}_{4}$, and $\mathrm{LTE}_{4}$ as demonstrated in rat lung fibroblasts. The role of cysLTs in collagen deposition is further verified by the dramatic reduction of airway collagen deposition by Monteleukast treatment in a chronic mouse asthma model, indicating a potent anti fibrotic pathway through cysL $\mathrm{T}_{1}$.

\section{Eicosanoids \& Fibrosis}

LTs and PGs work in opposition of one another to create a balance resulting in homeostasis. The overproduction of profibrotic and proinflammatory LTs and diminished $\mathrm{PGE}_{2}$ is associated with the development of fibrotic disorders. Evidence supporting the pivotal role for eicosanoids in fibrotic lung disease derives from observations that human and animal models of pulmonary fibrosis exhibit a synthetic imbalance favoring profibrotic LTs. The amount of $\mathrm{LTB}_{4}$ in bronchoalveolar lavage fluid (BAL) isolated from patients with IPF was greater than the amounts isolated from normal volunteer's samples [273-274]. Similarly, lung homogenates from IPF patients contained 15 fold greater $\mathrm{LTB}_{4}$ and 5 fold higher cysLTs that did normal controls [275]. Interestingly, the increased levels of LTs correlated directly with the histological severity of fibrosis, indicating a possible link between LTs and the progression of fibrosis. The source of LT overproduction are the AMs and is believed to be caused by constitutive activation of the 5LO enzyme. The observation of increased LT levels in the lung gathered from IPF patients correlated with the results of bleomycin induced fibrosis in both mice and hamsters, indicating the importance of LT expression in fibrotic disease.

The cause of increased LT synthesis in fibrotic lungs has not been identified, but a number of possible mediators are relevant candidates due to their elevated expression in the fibrotic lung and their ability to stimulate constitutive 5-LO activity. Candidates include 
cytokines, such as IL-8 [276], IL-4 [277] and IL-5 [278], immune complexes [279], MCP-1 [280], endothelin-1 [281] and TGF- $\beta$ [282-283]. Further studies are necessary to determine the stimulus and mechanism responsible for increased LT levels.

The underproduction of $\mathrm{PGE}_{2}$ diminishes any suppression of profibrotic signaling. This seems to be the case as determined from IPF patients and different animal models of fibrosis. BAL fluid from patients with IPF contains lower levels of $\mathrm{PGE}_{2}$ than BAL from controls. Fibroblasts isolate from IPF patient lung tissue demonstrate a reduced ability for $\mathrm{PGE}_{2}$ synthesis. The synthetic capacity by IPF fibroblasts is impaired under normal basal condition as well as in response to stimuli, such as LPS, IL-1, TNF- $\alpha$ and TGF- $\beta$. Impaired COX-2 mRNA and protein is responsible for the diminished synthetic activity. COX-2 is important in different inflammatory and induced settings; loss or diminished activity of this enzyme leaves cells unable to respond to their environment. Since $\mathrm{PGE}_{2}$ serves as an important counter signal to fibroblast proliferation and activation, the loss of its activity is a relevant and important component in fibrosis. 


\section{CHAPTER 4}

\section{Effects of Reovirus Exposure on Airway Reponses in an Experimental Model of Lower Respiratory Tract Infection (LRTI)}

\section{Rational}

Viral LRTI early in life has been reported as a potent initiating factor for the subsequent development of childhood asthma [1, 284-285]. Furthermore, LRTIs, such as viral bronchiolitis, are the leading cause of child and infant hospitalization, with an estimated 120,000 hospital admissions annually [286]. RSV is the predominant cause of severe bronchiolitis and pneumonia in infants worldwide [287]. In addition, there is growing evidence that early RSV infection is an important risk factor for the development of recurrent wheezing during the first decade of life [1]. Other common early life viral pathogens such as rhinovirus, influenza, parainfluenza (PIV), adenovirus, human metapneumovirus (hMPV), and human boca virus (hBoV) are known etiological agent of viral bronchiolitis [288-291]. Similarly, reoviruses are commonly found in the airways of exposed infants early in life [3-5] and are associated with pulmonary infections and the common cold [6]. The increased rate of reovirus infection during early childhood combined with the lack of information regarding the mechanisms behind its pathogenesis suggests that reovirus could be relevant in the development of childhood asthma.

Previous studies in animal models have shown that RSV infection results in dysregulated neuroimmune interactions and altered synthesis/release of pro-inflammatory neuropeptides generating amplified airway reactivity and neurogenic-mediated inflammation [284]. The essential and pivotal result of RSV infection is increased expression of Nerve Growth Factor (NGF) and its high affinity receptor tyrosine kinase A (TrKa) in the lungs [292]. NGF is the 
central regulatory factor controlling development, responsiveness and the level of neurotransmitter expression and transcription [293-294]. NGF increased expression and signaling results in augmented synthesis and release of the proinflammatory neurotransmitter substance $\mathrm{P}(\mathrm{SP})$ by nociceptive fibers of the lower respiratory tract [295]. Concomitantly, augmented NGF induces the over expression of SP's high-affinity receptor, neurokinin 1 (NK-1), by inflammatory cells and on the airway epithelium and vascular endothelium, resulting in exaggerated neurogenic inflammation [295]. Therefore, RSV-induced release of NGF primes different cellular effectors of the immune system (e.g., lymphocytes, monocytes, MCs), rendering them susceptible to the modulatory influence of the increased abundance of SP. The alterations to the airway sensory innervations induced by RSV infection early in life could predispose infants and children to the development of asthma.

The results of different studies indicate that the development of neurogenic inflammation is exceedingly dependent upon viral LRTI occurring early in life. Although to a lesser extent than RSV, reovirus exposure has been shown to be relevant within a critical time frame for the developing lung. Very little is known about the neurogenic-mediated airway responses following exposure to reovirus and their potential impact on the development of asthma later in life. Therefore, we wished to investigate the neurogenic inflammatory response to reovirus infection in an animal model of lung infection.

In addition, reovirus respiratory infection has been previously reported to generate increased collagen deposition and fibroblast proliferation. Specifically, Bellum et al. [38] reported that $\mathrm{CBA} / \mathrm{J}$ mice infected with reovirus $\mathrm{T} 1 / \mathrm{L}$ demonstrate a pathology of increased collagen deposition and fibroblast proliferation, making it an ideal animal model for BOOP. However, the processes and underlying mechanisms of fibrosis have not been clearly elucidated. 
Cav-1 and the eicosanoids, $\mathrm{PGE}_{2}$ and cysLTs, have been reported as principal mediators of fibrotic disease and may be important in the pathogenesis of reovirus induced fibrosis. Therefore, we investigated the effect of reovirus on airway remodeling and the relevance of cav1 and the eicosanoids reovirus pathogenesis.

Specific Aim 1: To investigate the effects of reovirus exposure on physiological airway responses and whether these responses were associated with neurogenic inflammation.

Specific Aim 2: To investigate the effects of reovirus exposure on expression neurotrophins and their receptors.

Specific Aim 3: To investigate the effects of reovirus exposure on airway remodeling.

\section{Material and Methods}

\section{$\underline{\text { Animals }}$}

Adult rats (12 week-old) of both sexes and weanling rats ( 2 week-old) born to pathogenfree timed pregnant dams of Fischer 344 (F-344) strain were obtained from Harlan Sprague Dawley Inc. (Indianapolis, IN). Due to the profound effects of respiratory infections on neurosensory airway regulation [296], the animals were kept in polycarbonate micro-isolator cages in a strictly controlled pathogen-free environment to prevent any microbial crosscontamination. These cages were placed on racks that provided positive individual ventilation with class 100 air to each cage at the rate of approximately one cage change of air per minute (Maxi-Miser; Thoren Caging System, Hazleton, PA). We used separate rooms for housing reovirus-infected and pathogen-free rats, serviced by trained husbandry technicians. All manipulations, inoculation and cage maintenance, were conducted inside class 100 laminar flow hoods. Bedding, water and food were autoclaved prior to use and unpacked only under laminar 
flow. Cages and water bottles were run through a tunnel washer after every use and disinfected with both chemical and heat. The West Virginia University Animal Care and Use Committee (WVU-ACUC) approved all experimental procedures followed in this study.

\section{Virus Preparation and Titration}

Reovirus was prepared according to a previously published method [297]. Briefly, reovirus serotype 1, strain Lang (T1L) stock, was originally obtained from Dr. Donald H. Rubin (Nashville, TN) and amplified in mouse L929 cells. Third-passage reovirus was purified by extraction with 1,1,2-trichloro-1,2,2-trifluoroethane followed by $\mathrm{CsCl}$ gradient centrifugation. The concentration of virions in the purified preparation was determined by measuring the optical density at $260 \mathrm{~nm}$ according to the method of Smith et al. [297]. The infectious viral dose was determined by plaque assay. Before inoculation, the virus stock was diluted for a final concentration of $2.5 \times 10^{6} \mathrm{PFU} / \mu 1$ (High dose) and $7.5 \times 10^{2} \mathrm{PFU} / \mu 1$ (Low dose). Virus-free media was used as a negative control.

\section{Inoculation}

Adult rats were anesthetized with pentobarbital sodium $(50 \mathrm{mg} / \mathrm{kg}$ i.p.). The trachea was carefully intubated with a 16-gauge cannula and $100 \mu 1$ of a suspension containing reovirus at either high dose or low dose or virus-free media was injected over the airway mucosa using $1 \mathrm{ml}$ syringe.

Weanling rats were anesthetized with pentobarbital sodium $(25 \mathrm{mg} / \mathrm{kg}$ i.p. $) .40 \mu \mathrm{lof}$ suspension containing reovirus at either high dose, low dose or virus-free media was carefully pipetted into the back of the weanling's throat and aspirated into the lungs. 


\section{Vascular Extravasation}

The extravasation of Evans Blue-labeled albumin from the tracheobronchial circulation was used as a marker of neurogenic-mediated inflammatory responses [298-299] following reovirus infection. Rats were anesthetized with pentobarbital sodium (adults: $50 \mathrm{mg} / \mathrm{kg}$ i.p.; weanlings: $25 \mathrm{mg} / \mathrm{kg}$ i.p.). The tracer, Evans blue dye $(30 \mathrm{mg} / \mathrm{kg}$ given i.v. over $5 \mathrm{sec}$.) was injected into the left femoral artery. Immediately after the injection of the tracer, virus infected and control rats were intravenously injected with $75 \mu \mathrm{g} / \mathrm{kg}$ of capsaicin (8-methyl-N-vanillyl-6nonenamide; Sigma-Aldrich, St. Louis MO) dissolved in a vehicle with a final concentration of $0.75 \%$ ethanol, $0.375 \%$ Tween 80 , and $0.85 \% \mathrm{NaCl}$ in aqueous solution over 2 min through the right femoral artery. The chemical was delivered in a volume of $1 \mathrm{ml} / \mathrm{kg}$ of body weight. Five minutes after injection of the tracer, the chest was opened and a 22-gauge cannula was inserted into the ascending aorta through the left ventricle. After incision of the left atrium, the circulation was perfused with $100 \mathrm{ml}$ of PBS (adult rats) or $50 \mathrm{ml}$ of PBS (weanling rats) over 2 min using a syringe pump. The extra pulmonary airways (from the first tracheal ring to the end of the main stem bronchi) and the left lung were dissected and prepared for Evans blue extraction. The connective tissue was carefully removed and the trachea was cut along the ventral side. The specimens were blotted dry, weighed and incubated in formamide for 18 hours at $50^{\circ} \mathrm{C}$ to allow the tracer to diffuse into the formamide. The extravasation of Evans bluelabeled albumin from the tracheobronchial circulation was quantified by measuring the optical density of the formamide extracts at a wavelength of $620 \mathrm{~nm}$. The quantity of Evans blue dye extravasated into the airway tissues, expressed in nanograms per milligram of wet-tissue weight, was interpolated from a standard curve of Evans blue concentrations $(0.5-10 \mu \mathrm{g} / \mathrm{ml})$. 


\section{Neurotrophin Immunoassay}

NGF and BDNF protein expression in the lungs were measured with a commercial kit (Promega, Madison, WI) using the antibody-sandwich technique. In brief, $100 \mathrm{mg}$ of lung-tissue samples were homogenized in 5 volumes of lysis buffer that contained $20 \mathrm{mM}$ Tris $\cdot \mathrm{HCl}$, $150 \mathrm{mM} \mathrm{NaCl}, 1 \%$ Nonidet P-40, 10\% glycerol, $1 \mathrm{mM}$ phenylmethylsulfonyl fluoride, $10 \mu \mathrm{g} / \mathrm{ml}$ aprotinin, $1 \mu \mathrm{g} / \mathrm{ml}$ leupeptin, and $0.5 \mathrm{mM}$ sodium vanadate. The supernatants of homogenized tissue samples were incubated for $18 \mathrm{~h}$ at $4^{\circ} \mathrm{C}$ in 96 -well plates coated with $100 \mu$ of either antiNGF or anti-BDNF polyclonal antibodies $(1.5 \mu \mathrm{g} / \mathrm{ml}$ in $100 \mathrm{mM}$ carbonate coating buffer; $\mathrm{pH}$ 9.7) to bind NGF or BDNF from the homogenates. After washing, a specific rat monoclonal antibody was applied $(0.6 \mu \mathrm{g} / \mathrm{ml}$ in buffer, $100 \mu \mathrm{l} /$ well $)$ and incubated for $18 \mathrm{~h}$ at $4^{\circ} \mathrm{C}$ to bind the captured NGF or BDNF. The plates were again thoroughly washed, and horseradish peroxidaseconjugated antibody to rat IgG was added to each well and incubated for $3 \mathrm{~h}$ at room temperature to detect the amount of specifically bound monoclonal antibody. After final washing to remove unbound antibody conjugate, the chromogenic substrate was added, and the color change generated by the reaction was read at a 450 -nm wavelength. Test samples and NGF or BDNF standards (100 $\mu 1 /$ well) were measured in duplicate. With this assay, NGF and BDNF can be quantified with a lower detection limit of $15.6 \mathrm{pg} / \mathrm{ml}$ and $<2 \%$ cross reactivity with other neurotrophic factors.

\section{Reverse Transcription-Polymerase Chain Reaction (RT-PCR)}

The presence of RNA in lung tissue was detected by RT-PCR in lung tissue specimens. The lungs were collected from the animals upon euthanasia with an i.p. dose of pentobarbital sodium followed by exsanguination. The tissue specimens were immediately flash frozen in liquid nitrogen and stored at $-80^{\circ} \mathrm{C}$. The frozen specimens were then placed in RLT disruption 
buffer and homogenized using a conventional rotor-stator homogenizer (Brinkmann Instruments, Westbury, NY) for 45-60sec until each sample was uniformly homogenous. Total RNA was extracted from lung homogenates using RNEasy Midi-Kits (Qiagen GmbH, Hilden, Germany) according to the manufacturer's specifications. RNA samples $(1 \mathrm{pg}-2 \mathrm{mg})$ were added to a $50 \mu 1$ master mix consisting of 400uM each of deoxynucleotide triphosphates (dNTPs), 10 units of RNAse inhibitor, $2 \mu$ of an enzyme mix containing Taq DNA Polymerase (one-step RT-PCR Promega, Madison, WI) and 50 pmol each of primers flanking the nucleotide sequence for NGF, BDNF, p75, TrKa, TrKb, and the housekeeping gene $\beta$-Actin. The same master mix without the RNA sample was used as a negative control. The primer pairs were designed on the basis of previously published protocols [300] and were used to differentiate cDNA-generated PCR products from genomic DNA contamination. The specific primer sequences (sense and antisense) are illustrated in table 1. Amplification was performed using a Gene-Amp PCR System 9600 thermal cycler (Perkin-Elmer, Waltham, MA). The process was started with an initial step of $50^{\circ} / 30$ min then $95^{\circ} / 15$ min followed by $25-35$ cycles with a denaturing step followed by an annealing step, an extension step, and then one final extension step at $68-72^{\circ}$ for 10 min. All programs included a $4^{\circ}$ hold step at the end. Amplified PCR products were sizefractionated by electrophoresis through a $2 \%$ agarose gel and stained with ethidium bromide. The gels were then photographed using an imaging system (FOTO/Analyst Luminary Workstation, Fotodyne, Hartland, WI). The intensity of DNA bands were analyzed by computerized densitometry (TotalLab TL-101 Image Analysis Software) and expressed as the ratio of the densitometric score measured for each target normalized by the $\beta$-actin control. The primer sequence (sense and antisense) for all gene targets and their expected size is shown in Table 1. 


\section{Histopathology}

The right lung from each animal was fixed in $10 \%$ buffered formalin, embedded in paraffin, and cut in 3-mm-thick sections. Hematoxylin and Eosin (H\&E) and Masons Trichrome staining was performed for histopathologic analysis. All slides were coded and interpreted by two independent pathologists who were blinded to whether the specimens were coming from an infected animal or a control. Histopathologic changes were graded as mild (5-10 inflammatory cells in 3 or more adjacent alveoli), moderate (10-20 inflammatory cells per alveolus), or severe (>20 inflammatory cells per alveolus).

Morphometric analysis was conducted on Trichrome-stained slides to quantitate the presence of collagen. Regions of interest for each slide were identified based on the presence of the same pulmonary structures. The quantity of collagen within the selected field was determined by computerized analysis of the intensity of blue-stained structures and expressed as a percentage of tissue area.

\section{$\underline{\text { Cav-1 Western Blot }}$}

The lungs were collected from the animals upon euthanasia with an i.p. dose of pentobarbital sodium followed by exsanguination. The tissue specimens were immediately flash frozen in liquid nitrogen and stored at $-80^{\circ} \mathrm{C}$. The frozen specimens were then placed in homogenization buffer $\left(0.1 \mathrm{M}\right.$ Phosphate $\left(\mathrm{PO}_{4}\right) \mathrm{pH} 7.4$ contained $1 \mathrm{mM}$ EDTA and $10 \mu \mathrm{M}$ indomethacin) and homogenized using a conventional rotor-stator homogenizer (Brinkmann Instruments, Westbury, NY) for 45-60sec until each sample was uniformly homogenous. Sample protein concentration was determined using a BCA protein assay kit purchased from Pierce (Rockford, IL). Based on protein concentration, homogenates were diluted to $20 \mu \mathrm{g}$ of protein/sample. Each sample received SDS sample buffer containing 1M Tris- $\mathrm{HCl}(\mathrm{pH} 6.8), 5 \%$ 
(w/v) SDS, 2.5\% (v/v) $\beta$-mercaptoethanol, 5\% glycerol in double-distilled water and boiled to solubilize sample proteins. Boiled samples were subjected to SDS/PAGE, electrotransferred to a nitrocellulose membrane, and immunoblotted with Cav-1 and $\beta$-actin monoclonal antibodies from BD Biosciences (San Diego California). Bands were visualized using Super Signal West Pico Stable Peroxide and Luminol/Enhancer solutions (Pierce). Densitometry analysis of blots and corresponding $\beta$-actin controls was performed using an imaging system (FOTO/Analyst Luminary Workstation, Fotodyne, Hartland, WI). The intensity of protein bands was analyzed by computerized densitometry (TotalLab TL-101 Image Analysis Software) and expressed as the ratio of the densitometric score measured for each target normalized by the $\beta$-actin control.

\section{Quantification of $\mathrm{PGE}_{2}$ by enzyme immunoassay (EIA)}

$\mathrm{PGE}_{2}$ was extracted from lung homogenates by solid phase extraction with $\mathrm{C} 18$ solid phase extraction cartridges (SPE Cartridges/C-18) purchased from Caymen Chemical (Ann Arbor, MI). Post removal, lung tissue samples were flash frozen in liquid nitrogen and stored at $-80^{\circ} \mathrm{C}$. The frozen specimens were then placed in homogenization buffer $\left(0.1 \mathrm{M}\right.$ Phosphate $\left(\mathrm{PO}_{4}\right)$ pH 7.4 contained $1 \mathrm{mM}$ EDTA and $10 \mu \mathrm{M}$ indomethacin) and homogenized using a bead beater. Homogenized samples were diluted 1:4 in homogenization buffer and the $\mathrm{pH}$ of samples was measured and adjusted to 4.0 using $\mathrm{HCl}$. $\mathrm{pH}$ adjusted samples were applied to the $\mathrm{C} 18$ cartridge (pretreated with methanol followed by HPLC grade water). $\mathrm{PGE}_{2}$ was eluted from the cartridge with ethyl acetate containing 1\% methanol. Eluted samples in methanol were dried with a speedvac concentrator under vacuum and reconstituted with enzyme immunoassay (EIA) buffer from Caymen Chemicals (Ann Arbor, MI) for EIA measurement. The concentration of $\mathrm{PGE}_{2}$ in lung homogenate samples were analyzed using a specific EIA kit from Caymen Chemicals (Ann Arbor, MI) according to manufacturer's instructions. 


\section{Quantification of CysLT by enzyme immunoassay (EIA)}

cysLTs were isolated from long homogenates using a cysLT Affinity Purification Kit from Caymen Chemicals (Ann Arbor, MI) per manufacturer's instructions. The concentrations of CysLTs in lung homogenates were evaluated using a specific EIA kit from Caymen Chemicals (Ann Arbor, MI) according to manufacturer's instructions.

\section{$\underline{\text { Statistical Analysis }}$}

All data were analyzed using a multivariate analysis of variance (ANOVA) for repeated measures. If a significant effect was found, a post-hoc t-test with Bonferroni correction was used to identify significant pairs. Individual comparisons were made using paired and unpaired t-test when appropriate (Sigmastat 2.0 for Windows, SPSS Inc., Chicago, IL). Values in the text and figures are presented as mean $\pm \mathrm{SE}$. Differences having a $\mathrm{P}$ value $<0.05$ were considered significant.

\section{Experimental Protocols}

\section{Specific Aim 1 \& 2}

a) 5-Day Protocol. Adult rats (12 week-old) were infected with $100 \mu 1$ of a suspension containing either high dose reovirus $\left(2.5 \times 10^{6} \mathrm{PFU} / \mu \mathrm{l} ; \mathrm{n}=6\right)$ or low dose reovirus $\left(7.5 \times 10^{2}\right.$ $\mathrm{PFU} / \mu 1 ; \mathrm{n}=6$ ). Control animals $(\mathrm{n}=5)$ were inoculated with an equal volume of virus-free media. Weanling rats ( 2 week-old) were infected with $40 \mu 1$ of a suspension containing either high dose reovirus $\left(2.5 \times 10^{6} \mathrm{PFU} / \mu \mathrm{l} ; \mathrm{n}=5\right)$ or low dose reovirus $\left(7.5 \times 10^{2} \mathrm{PFU} / \mu \mathrm{l} ; \mathrm{n}=6\right)$. Control animals $(n=5)$ were inoculated with an equal volume of virus-free media. Five days after inoculation, the Evans Blue procedure was performed as previously described. The extra pulmonary airways (from the first tracheal ring to the end of the main stem bronchi) and the left lung were dissected 
and prepared for Evans blue extraction. The right lung was removed for RNA extraction and histopathologic analysis. All animals were weighed before inoculation and after the 5-day incubation period.

b) 30-Day Protocol. Adult rats (12 week-old) were infected with $100 \mu 1$ of a suspension containing either high dose reovirus $\left(2.5 \times 10^{6} \mathrm{PFU} / \mu 1 ; \mathrm{n}=6\right)$ or low dose reovirus $\left(7.5 \times 10^{2}\right.$ PFU/ $\mu 1 ; n=6)$. Control animals $(n=6)$ were inoculated with an equal volume of virus-free media. Weanling rats ( 2 week-old) were infected with $40 \mu 1$ of a suspension containing either high dose reovirus $\left(2.5 \times 10^{6} \mathrm{PFU} / \mu \mathrm{l} ; \mathrm{n}=6\right)$ or low dose reovirus $\left(7.5 \times 10^{2} \mathrm{PFU} / \mu \mathrm{l} ; \mathrm{n}=6\right)$. Control animals $(n=6)$ were inoculated with an equal volume of virus-free media. Thirty days after inoculation, the Evans Blue procedure was performed as previously described. The extra pulmonary airways (from the first tracheal ring to the end of the main stem bronchi) and the left lung were dissected and prepared for Evans blue extraction. The right lung was removed for RNA extraction and histopathologic analysis. All animals were weighed before inoculation and after the 30-day incubation period.

\section{Specific Aim 3}

5-Day protocol: Weanling rats (2 week-old) were infected with $40 \mu \mathrm{l}$ of a suspension containing either high dose reovirus $\left(2.5 \times 10^{6} \mathrm{PFU} / \mu \mathrm{l} ; \mathrm{n}=2\right)$ or low dose reovirus $\left(7.5 \times 10^{2}\right.$ PFU $/ \mu 1 ; n=2)$ and control animals $(n=2)$ were inoculated with an equal volume of virus-free media. At 5 days post inoculation rats were anesthetized with Pentobarbital sodium $(25 \mathrm{mg} / \mathrm{kg}$ i.p.). Blood samples were taken prior to sacrifice. Lungs were inflated with .5 to $1.5 \mathrm{ml}$ of formalin using a 16 gauge cannula attached to a syringe and allowed to sit for 5 minutes prior to removal. The right lung was dissected and prepared for histopathologic analysis. The left lung was removed for RNA, protein and hormone analysis. 
60-Day protocol: Weanling rats ( 2 week-old) were infected with $40 \mu 1$ of a suspension containing either high dose reovirus $\left(2.5 \times 10^{6} \mathrm{PFU} / \mu \mathrm{l} ; \mathrm{n}=2\right)$ or low dose reovirus $\left(7.5 \times 10^{2}\right.$ $\mathrm{PFU} / \mu 1 ; n=3)$. Control animals $(\mathrm{n}=3)$ were inoculated with an equal volume of virus-free media. At 60 days post inoculation rats were anesthetized with Pentobarbital Sodium (25mg/kg i.p.). Blood samples were taken prior to sacrifice. Lungs were inflated with .5 to $1.5 \mathrm{ml}$ of formalin using a 16 gauge cannula attached to a syringe and allowed to sit for 5 minutes prior to removal. The right lung was dissected and prepared for histopathologic analysis. The right lung was removed for RNA, protein and hormone analysis.

90-Day protocol: Weanling rats ( 2 week-old) were infected with $40 \mu 1$ of a suspension containing either high dose reovirus $\left(2.5 \times 10^{6} \mathrm{PFU} / \mu \mathrm{l} ; \mathrm{n}=2\right)$ or low dose reovirus $\left(7.5 \times 10^{2}\right.$ $\mathrm{PFU} / \mu 1 ; n=3)$. Control animals $(\mathrm{n}=2)$ were inoculated with an equal volume of virus-free media. At 90 days post inoculation rats were anesthetized with Pentobarbital Sodium (50mg/kg i.p.). Blood samples were taken prior to sacrifice. Lungs were inflated with .5 to $1.5 \mathrm{ml}$ of formalin using a 16 gauge cannula attached to a syringe and allowed to sit for 5 minutes prior to removal. The right lung was dissected and prepared for histopathologic analysis. The right lung was removed for RNA, protein and hormone analysis.

120-Day protocol: Weanling rats (2 week-old) were infected with $40 \mu 1$ of a suspension containing either high dose reovirus $\left(2.5 \times 10^{6} \mathrm{PFU} / \mu 1 ; \mathrm{n}=2\right)$ or low dose reovirus $\left(7.5 \times 10^{2}\right.$ $\mathrm{PFU} / \mu \mathrm{l} ; \mathrm{n}=3$ ). Control animals $(\mathrm{n}=2)$ were inoculated with an equal volume of virus-free media. At 120 days post inoculation rats were anesthetized with Pentobarbital Sodium (50mg/kg i.p.). Blood samples were taken prior to sacrifice. Lungs were inflated with .5 to $1.5 \mathrm{ml}$ of formalin using a 16 gauge cannula attached to a syringe and allowed to sit for 5 minutes prior to removal. 
The right lung was dissected and prepared for histopathologic analysis. The right lung was removed for RNA, protein and hormone analysis.

\section{Results}

\section{HISTOPATHOLOGY AND MORPHOMETRIC ANALYSIS}

\section{Weanlings}

H\&E stained microscopic lung sections from weanling rats sacrificed 5 days post inoculation were analyzed to determine the inflammatory response to reovirus and how it is augmented by virus dose. Rats infected with high dose reovirus developed multifocal and coalescent, acute, suppurative and lymphocytic bronchopneumonia. The grade of bronchopneumonia was described as moderate to acute. Features of the pneumonia included the accumulation of neutrophils in the alveolar region, intraluminal accumulation of mucus in bronchioles, and peribronchiolar and perivascular accumulation of mononuclear inflammatory cells (Figure 11-A \& Figure 12-A). In comparison, the Low dose reovirus resulted in a milder multifocal, lymphocytic, bronchopneumonia. Features of the pneumonia included the peribronchiolar and perivascular accumulation of mononuclear inflammatory cells that were predominantly lymphocytes (Figure 11-B Figure 12-A). Control animals demonstrated no significant findings. 
A.

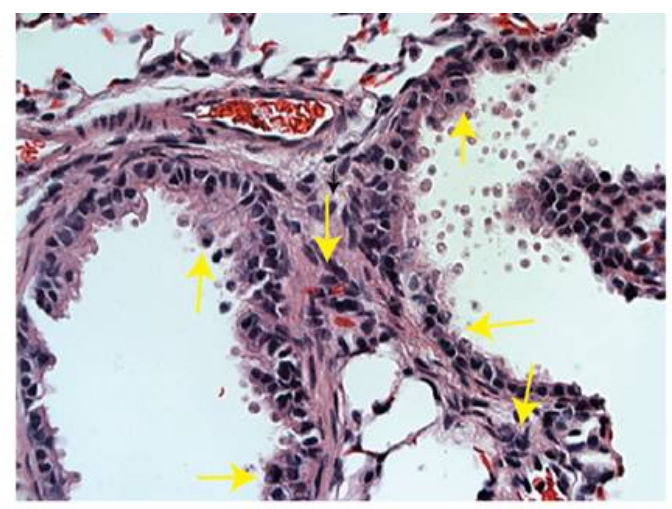

C.

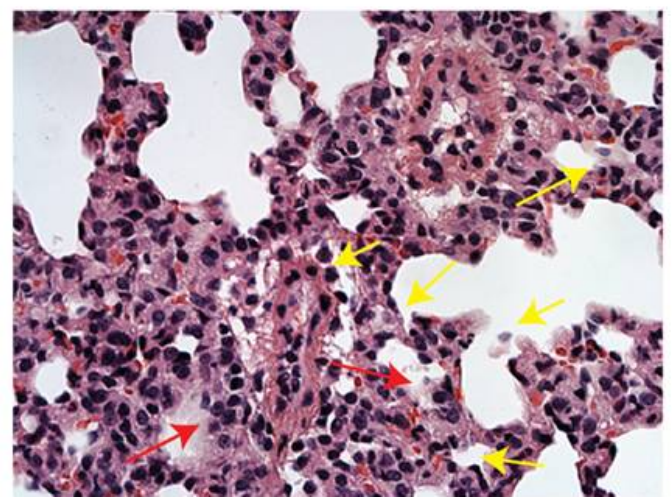

B.

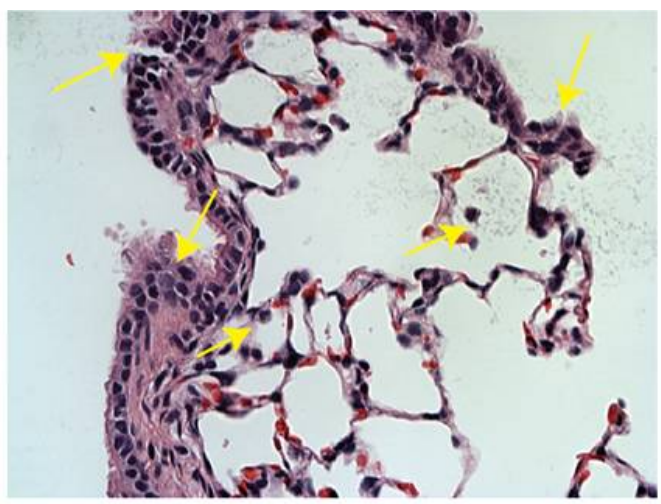

D.

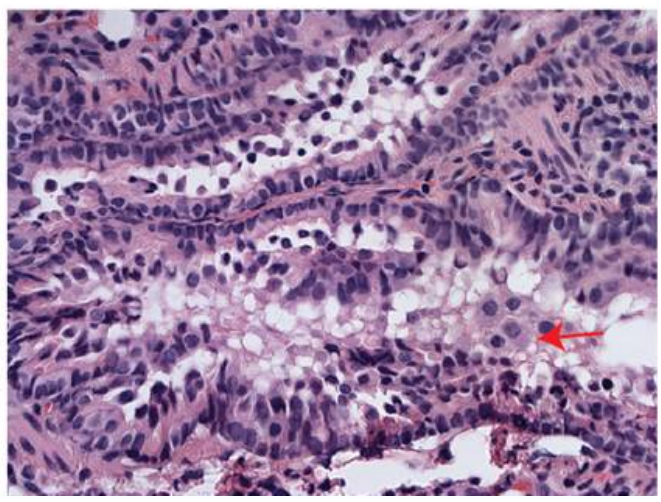

Figure 11 Histological features of reovirus induced pneumonia at 5 and 30 days post infection

Electron micrographs of H\&E stained lung sections from weanling rats infected with either 2.5x106 PFU/ $\mu$ l (High dose) or $7.5 \times 102 \mathrm{PFU} / \mu \mathrm{l}$ (Low dose) of reovirus T1/L at $40 \mathrm{x}$ magnifications. A) The accumulation of polymorphonuclear cells (PMNs) in the alveolar spaces (yellow arrows), perivascular accumulation of mononuclear inflammatory cells, intralumenal accumulation of mucous in bronchioles, and peribronchiolar influx of neutrophils was apparent in lung sections from rats 5 days post infection with high dose reovirus. B)Low dose virus infection resulted in peribronchiolar and perivascular accumulation of mononuclear inflammatory cells (yellow arrows) at 5 days post infection. C) PMNs (yellow arrows) and chronic inflammatory cells were seen within the bronchioles and the interstitium at 30 days post infection with high dose virus. Mucus and necrotic cellular debris was also noted in bronchiole spaces as well as foamy macrophages (red arrows). D)Low dose reovirus infection resulted in mild chronic peribronchiolar inflammation. Features included mild, multifocal, mucous metaplasia, presence of chronic inflammatory cells in the interstitium and around respiratory bronchioles, with foamy macrophages being seen as well (red arrow).

At 30 days post inoculation rats infected with high dose virus exhibited moderate chronic inflammation that was focused around respiratory bronchioles. Inflammatory features included the presence of PMNs and chronic inflammatory cells within the bronchioles and the interstitium (Figure 12-A). Mucus and necrotic cellular debris was also found in bronchiole spaces along with foamy macrophages (Figure 11-C). In a few samples, occasional bronchioles contained detached epithelial cells associated with faint cytoplasmic vacuoles and basophilic foci, which could be representative of viral inclusions. Low dose reovirus infection resulted in mild chronic 
peribronchiolar inflammation. Features included mild, multifocal, mucus metaplasia, presence of chronic inflammatory cells in the interstitium and around respiratory bronchioles (Figure 12-

A). Moderate, multifocal, medial hypertrophy of pulmonary arterioles was also identified and specific to low dose virus infection.

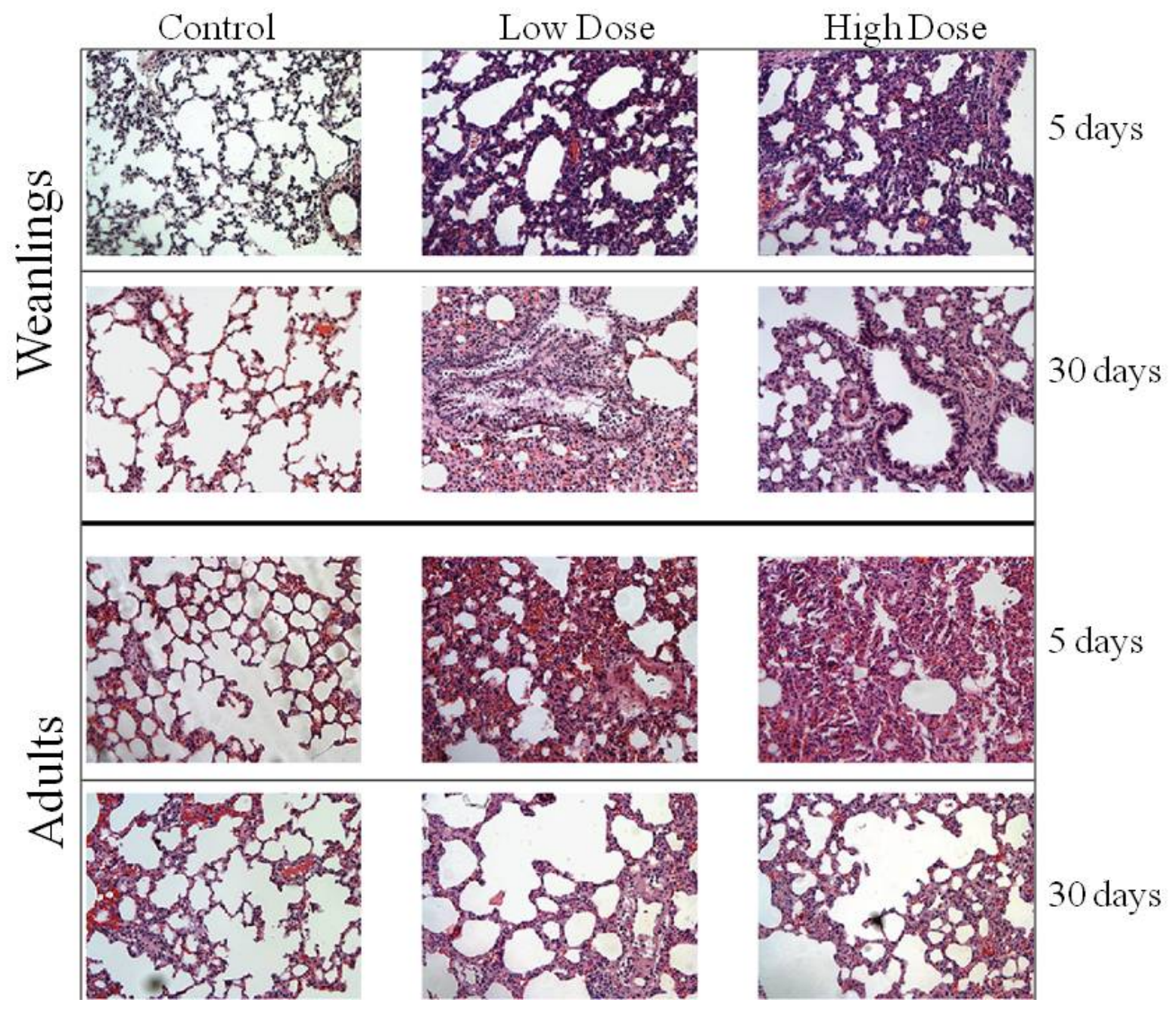

Figure 12 Histological features of reovirus induced pneumonia in adult and weanling rats

Electron micrographs of H\&E stained lung sections from weanling rats infected with either $2.5 \times 106 \mathrm{PFU} / \mu 1$ (High dose) or $7.5 \times 102 \mathrm{PFU} / \mu \mathrm{l}$ (Low dose) of reovirus $\mathrm{T} 1 / \mathrm{L}$ at $40 \mathrm{x}$ magnifications. A) H\&E-stained microscopic sections of weanling rat lungs obtained 5 and 30 days following inoculation with either low or high dose reovirus or pathogen-free vehicle. At 5 days postinfection, lung sections from rats infected with either dose of reovirus, show acute cellular infiltrates consisting mainly of neutrophils, monocytes and mast cells consistent with moderate to severe interstitial and peribronchial inflammation compared to control animals. At 30 days the acute histopathologic changes were completely resolved and no significant differences were observable between treated and virus-free animals. Images are representative of 6 animals. B) H\&E-stained microscopic sections of adult rat lungs obtained 5 and 30 days post inoculation. Five days following infection with either dose of reovirus, the animals developed an acute inflammatory response characterized by the presence of moderate to severe acute peribronchial and interstitial cellular infiltrates comprised of neutrophils and monocytes which was associated with mild hyperplasia of bronchus-associated lymphoid tissue (BALT). These changes were lost at 30 days post inoculation as shown by the lack of any inflammatory cells in either virus-infected or pathogen-free animals. Images are representative of 6 animals. 
Trichrome stained microscopic lung sections from weanling rats 30 days post reovirus infection were analyzed for changes in collagen deposition in response to reovirus infection. Overall, reovirus infected weanlings exhibited signs of increased collagen accumulation associated with the early stages of fibrosis development at 30 days post infection. High dose reovirus infection resulted in focal, mild fibrosis (Figure 13-A). Fibrotic regions demonstrated decreased vascular perfusion in conjunction with increases in epithelial cells. Areas demonstrating intensified blue staining exhibited augmented collagen accumulation associated with increased numbers of fibroblasts in terminal respiratory bronchioles and alveolar spaces of the interstitium suggestive of interstitial fibrosis. Low dose reovirus infection resulted in a similar pathology of focal, mild fibrosis (Figure 13-A). Overall, the Trichrome-stained microscopic sections of weanling rat lungs 30 days after infection, showed signs of collagen accumulation which was graded as mild fibrosis.

Computerized morphometric analysis of lung sections showed a small, yet significant increase of collagen content in the lungs of low dose treated rats compared to controls (Figure $13-\mathrm{B})$ with a $\mathrm{p}$ value $<0.05$. A more robust increase in collagen was seen in response to high dose virus infection as demonstrated by a more significant increase in collagen content in infected rats compared to controls with a $\mathrm{p}$ value $<0.001$. These changes were not observed in any of the animals 5 days after infection. 

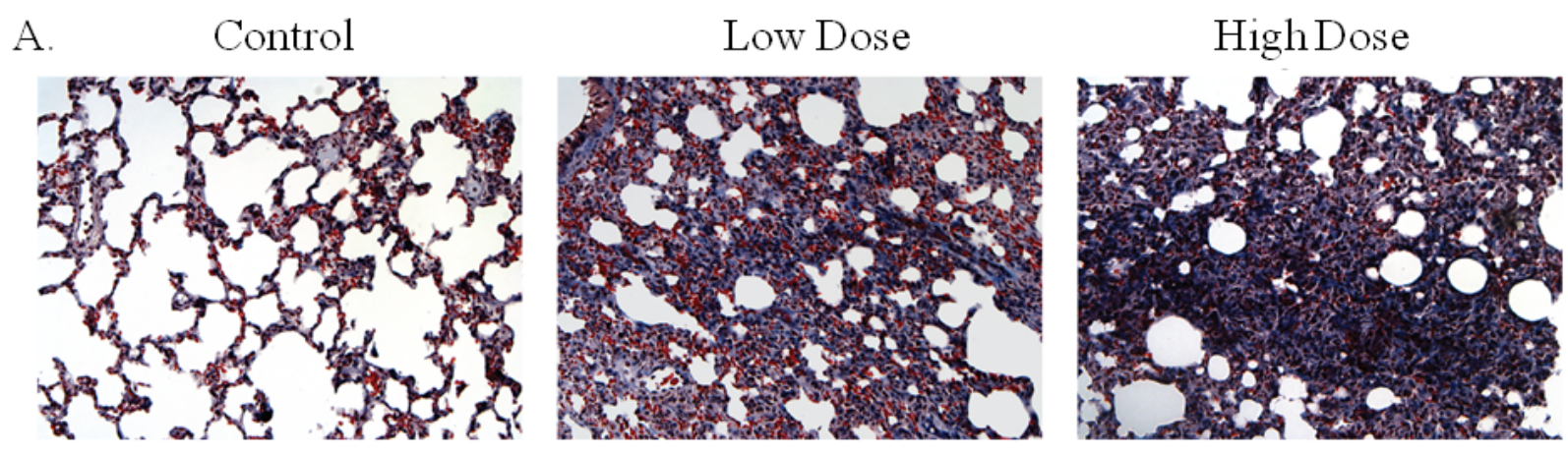

B.

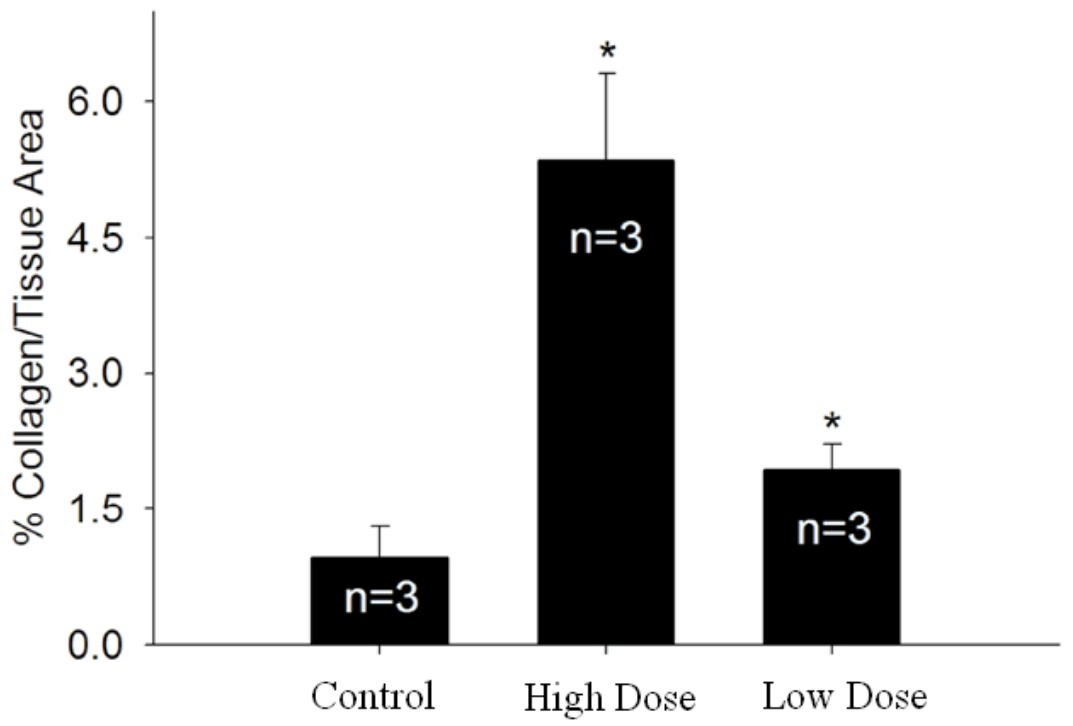

Figure 13 Trichrome Staining and Morphometric Analysis

A) Masson's Trichrome-stained weanling rat lung sections obtained 30 days after inoculation with either dose of reovirus or virus-free vehicle. An increased degree of collagen deposition was observed in infected rats compared to age-matched pathogenfree controls as indicated by a more significant blue staining Images are magnified 20x and are each representative of 6 animals. B) The computerized morphometric analysis of the same lung sections showed a significantly higher collagen deposition in treated animals compared to controls. This effect appears to be dose-dependent. Data are expressed as \%collagen/ tissue area and are mean $+/-\mathrm{SE}\left(\mathrm{n}=6 ;{ }^{* * *} \mathrm{p}<0.001 ;{ }^{*} \mathrm{p}<0.05\right.$; ANOVA with Fisher LSD post-hoc analysis).

At 60 days post inoculation rats infected with either reovirus dose exhibited mild focal chronic inflammation (Figure 14). Chronic inflammation was mainly focused on respiratory bronchioles. Germinal centers around airway spaces were observed. No acute inflammatory cells were observed, but a few foamy macrophages were seen in the lumen of terminal respiratory bronchioles. Trichrome staining revealed focal increases in collagen deposition as 
well as a thickening of alveoli septa. Increased number of fibroblasts associated with respiratory bronchioles was noted.
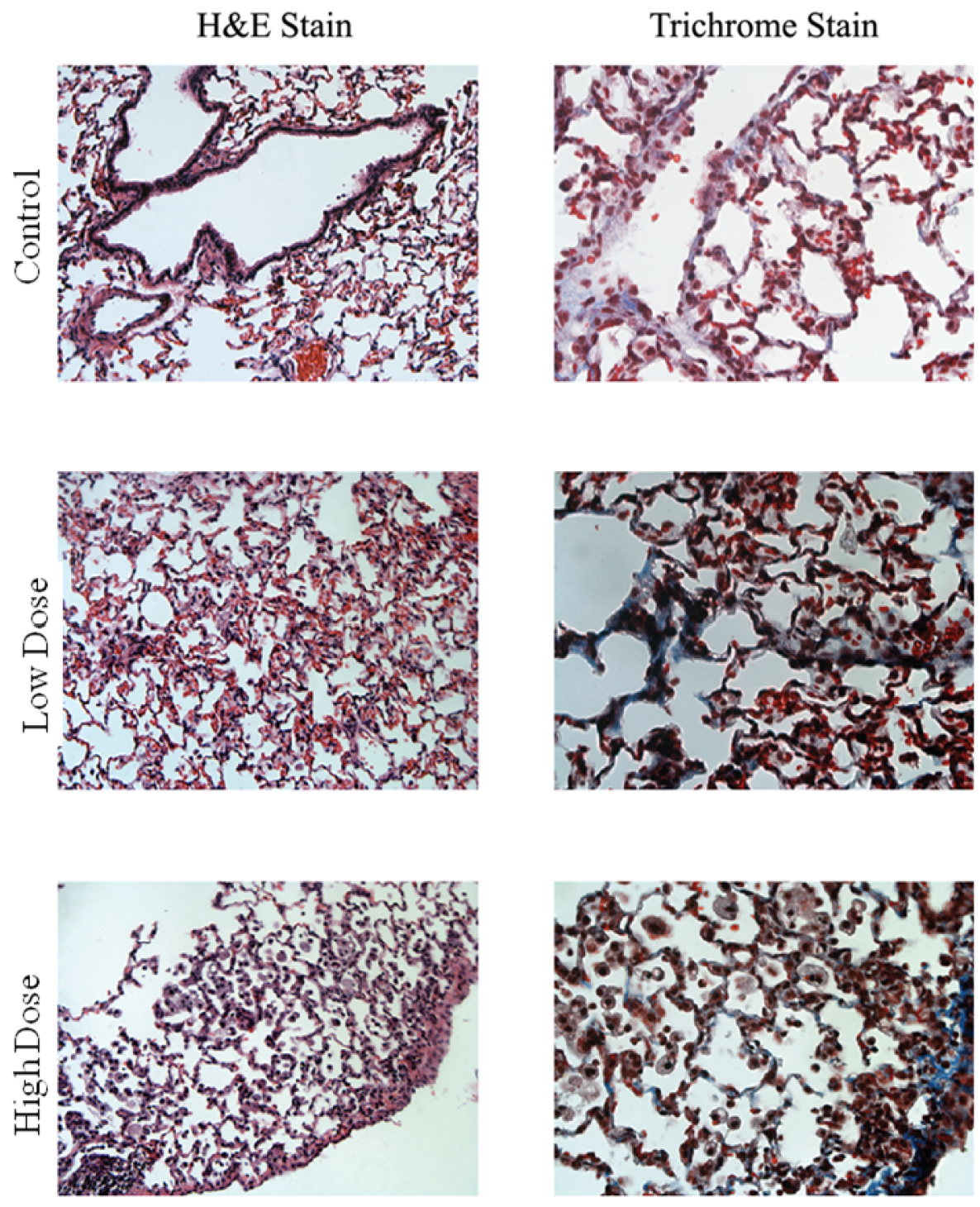

\section{Figure 14 Histology at 60 days post infection}

Electron micrographs of lung sections from weanling rats infected with either $2.5 \times 10^{6} \mathrm{PFU} / \mu \mathrm{l}$ or $7.5 \times 10^{2} \mathrm{PFU} / \mu \mathrm{l}$ of reovirus T1/L at 20× magnification.H\&E and Trichrome stained microscopic sections of rat lungs obtained 60 days post inoculation. Chronic inflammation was mainly focused on respiratory bronchioles. Germinal centers around airway spaces are observed. No acute inflammatory cells were observed, but a few foamy macrophages were seen in the lumen of terminal respiratory bronchioles. 


\section{Adults}

Five days post reovirus infection, H\&E-stained microscopic sections demonstrated acute peribronchial and interstitial cellular infiltrates made of neutrophils and monocytes which were graded moderate to severe (Figure 12). In addition, the presence of locally extensive, mild hyperplasia of bronchus-associated lymphoid tissue (BALT) was noted (Figure 15). These inflammatory cellular infiltrates were minimal or absent 30 days post reovirus infection (Figure 12). The lungs of control rats showed minimal or no pathologic changes. No significant differences were observed in the severity of pathologic changes between rats infected with high or low dose reovirus.

Control

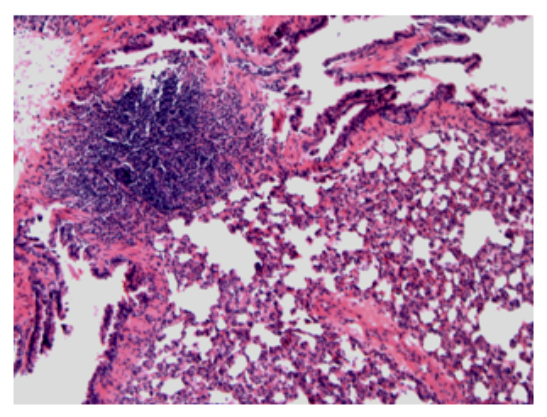

Low Dose

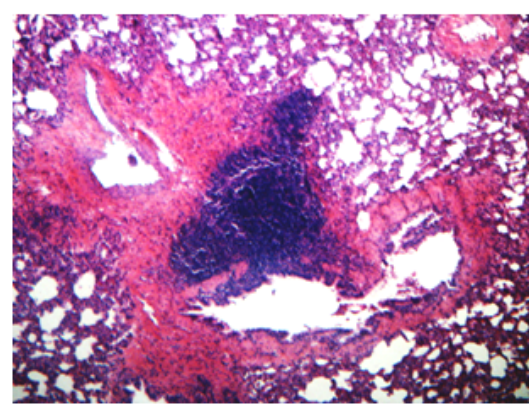

High Dose

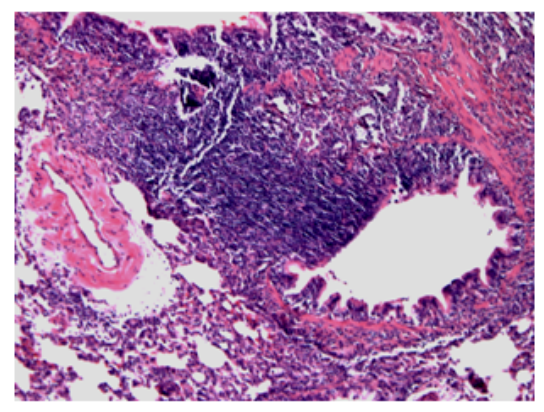

Figure 15 Adult Rat Bronchus-associated Lymphoid Tissue (BALT)

H\&E stained microscopic sections of adult rat lungs 5 days post reovirus infection. Locally extensive, mild hyperplasia of BALT was associated with reovirus infection. Low dose infection results in the expansion of BALT as demonstrated by the increased infiltration by inflammatory cells into the bronchiole epithelium and interstitium. High dose induced a similar hyperplasia with accumulations of lymphoid cells and formation of lymphoid follicles in the interstitium that expanded into the bronchiole lumen. Both low and high dose showed active germinal centers.

\section{Body Weight}

In order to further document the pathologic effect of the virus, we measured the average weight gain between inoculation and the end of incubation time, immediately before performing the vascular extravasation procedure. Weight gain was significantly reduced in weanling rats five 
days after infection compared to controls. Weight gain was unaffected at 30 days post infection as well as in treated and control adult rats at 5 and 30 days (Figure 16).

A.
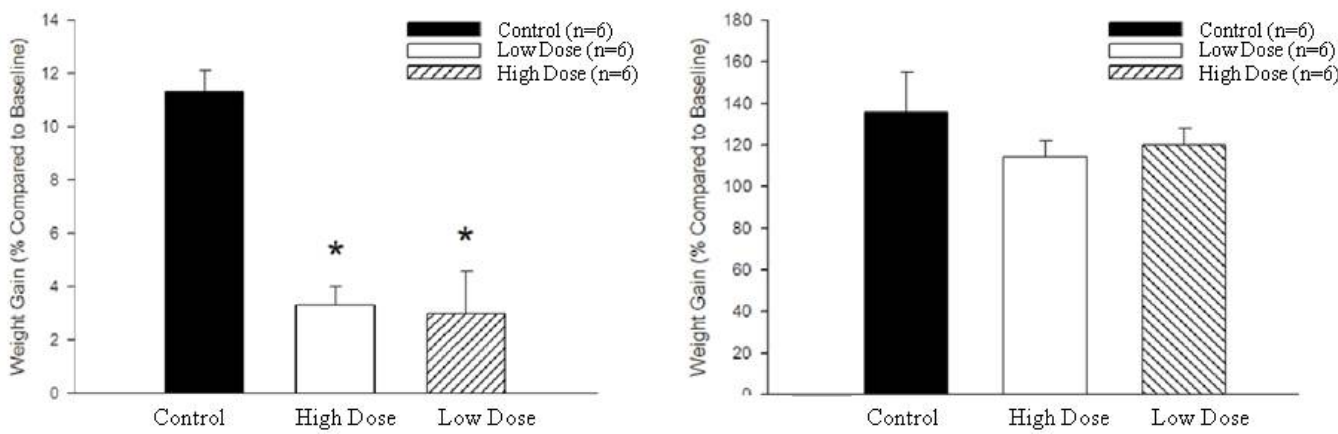

B.
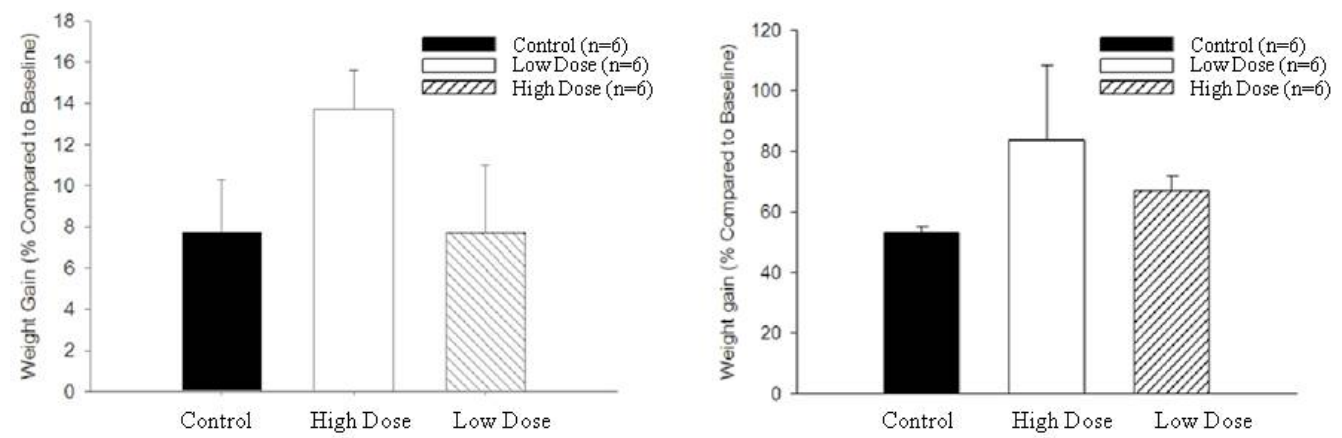

Figure 16 Acute Changes in Body Weight Demonstrate Reovirus Pathology

A) Weanlings failed to gain weight during the 5 days following infection with either low or high reovirus dose compared to their age-matched pathogen-free controls. Data are expressed as the average weight gain (\% compared to pre-treatment value) and are indicated as mean $\pm \mathrm{SE}(\mathrm{n}=6 ; * * * \mathrm{p}<0.001$; ANOVA with Fisher LSD post-hoc analysis). B) The growth rate was restored during the 30 days after reovirus infection and was comparable to pathogen-free controls. Data are expressed as the average weight gain (\% compared to pre-treatment value) and are indicated as mean $\pm \mathrm{SE}\left(\mathrm{n}=6 ;{ }^{* * *} \mathrm{p}<0.001\right.$; ANOVA with Fisher LSD post-hoc analysis). C) In adult rats, at 5 days post infection, no significant changes in weight gain were observed in infected rats compared to pathogen-free controls. Data are expressed as the average weight gain (\% compared to pre-treatment value) and are indicated as mean $\pm \mathrm{SE}(\mathrm{n}=6 ; \mathrm{p}=\mathrm{ns}$; ANOVA). D) In adult rats, at 30 days post infection, no significant changes in weight gain were observed in infected rats compared to pathogen-free controls. Data are expressed as the average weight gain (\% compared to pretreatment value) and are indicated as mean $\pm \mathrm{SE}(\mathrm{n}=6 ; \mathrm{p}=\mathrm{ns}$; ANOVA).

\section{Vascular Permeability}

In adult rats, Evans blue-labeled albumin extravasation from extra pulmonary and intra pulmonary airways was significantly increased 5 days after inoculation compared to agematched controls (Figure 17-C). The magnitude of the increase was not significantly different 
between high and low dose reovirus-treated animals. This effect was still significant 30 days after infection in the animals treated with the higher dose only (Figure 17-D).

In weanling rats, vascular extravasation from the lung was significantly larger in low dose infected rats compared to controls (Figure 17-A). Evans-blue labeled extravasation from the trachea was unaffected at 5 days. These effects were lost 30 days after reovirus infection (Figure 17-B).
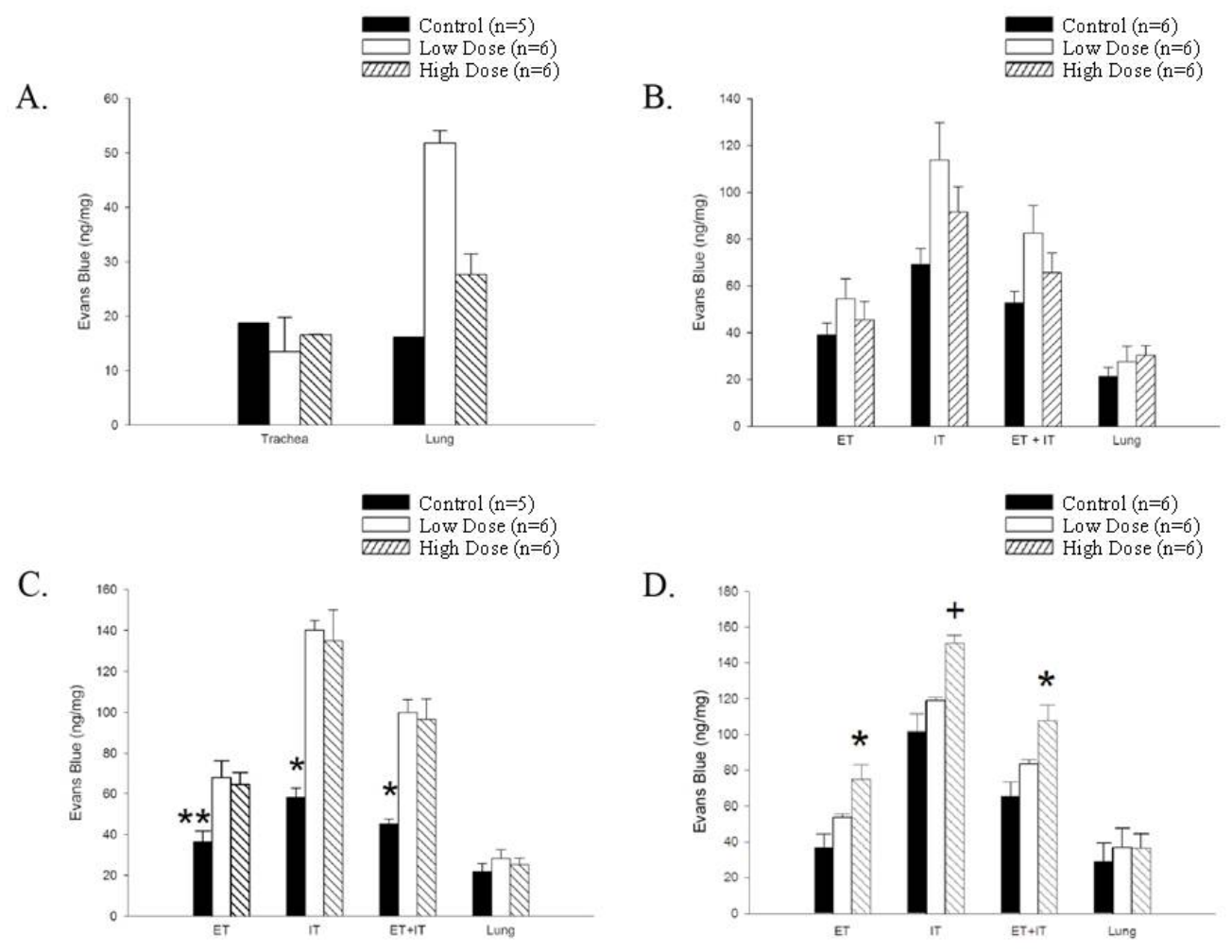

Figure 17 Changes in Vascular Permeability indicative of Neurogenic Inflammation

A) Extravasation of Evans blue-labeled albumin from the airway microvasculature in weanling rats following inoculation with either low or high dose reovirus or pathogen-free vehicle. In the intrapulmonary airways, vascular extravasation was significantly higher in infected animals compared to pathogen-free controls. This effect appears to be dose-related and is lost 30 days after inoculation. The extrapulmonary airway vessels were unaffected. Data are expressed as mean $\pm \mathrm{SE}(\mathrm{n}=5-6$; ***p $<0.001 \mathrm{vs}$ controls and low dose; \#p<0.001 vs controls and high dose; ANOVA with Fisher LSD post-hoc analysis). B) Vascular extravasation in the intrapulmonary and extrapulmonary airway microcirculation of adult rats 5 and 30 days after treatment with either dose of reovirus or pathogen-free vehicle. At 5 days post inoculation the quantity of Evans blue-labeled albumin recovered from the extrapulmonary airway micro vessels was significantly higher in infected rats compared to pathogen-free controls. No differential effects were observed between low and high reovirus dose. In animals infected with high dose reovirus, the increased vascular permeability was still significantly higher in treated animals compared to controls 30 days post reovirus infection. Data are expressed as mean $\pm \mathrm{SE}(\mathrm{n}=5-6 ; * * * \mathrm{p}<0.001$; ANOVA with Fisher LSD post-hoc analysis $)$. 


\section{Neurotrophin Expression}

\section{Protein Expression}

In order to determine if the changes in vascular extravasation seen in response to reovirus infection were mediated by neurogenic mechanisms we analyzed the protein expression of BDNF and NGF. In weanling rats NGF protein expression (Figure 18-B) was increased in control and low dose animal at 5 days post infections when compared to high dose animals which showed diminished NGF. These differences were not significant. The amount of NGF protein decreased at 30 days and was not affected by virus infection with all experimental groups showing similar expression. BDNF in weanling rats showed no significant changes at 5 and 30 days post infection, though there were higher levels of BDNF post viral clearance (Figure 18-A). In adult rats the amount of NGF (Figure 18-D) and BDNF (Figure 18-C) protein was unaffected by reovirus infection at both 5 and 30 days. 

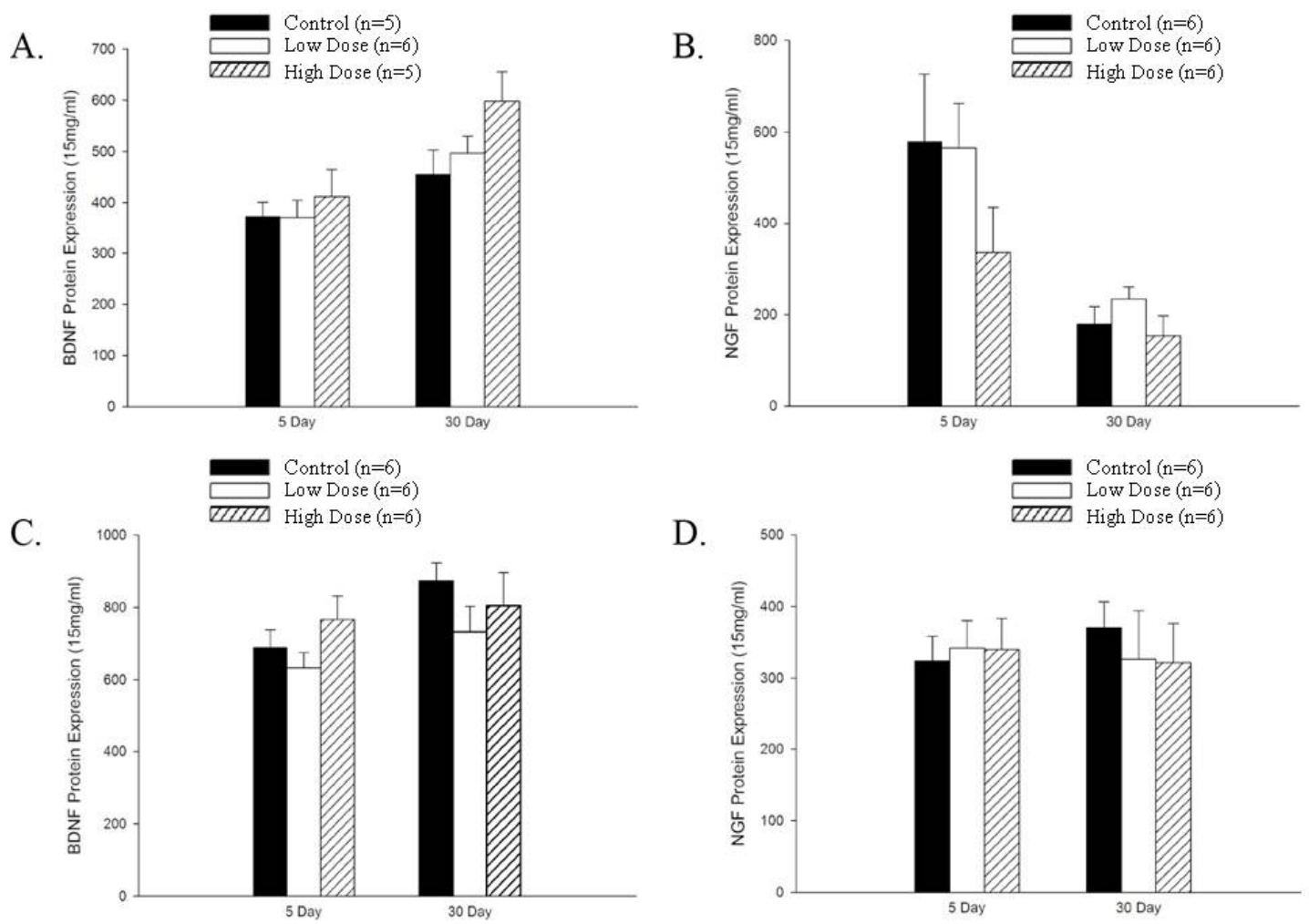

Figure 18 Neurotrophin Protein Expression at 5 \& 30 days

The amount of NGF and BDNF protein in rat lung homogenates were analyzed by ELISA at 5 and 30 days post infection. A) BDNF protein in weanling rats showed no significant changes at 5 and 30 days post infection. B) NGF protein expression in weanling rats at 5 days post infection with low and high dose reovirus demonstrated increased amounts of NGF protein. The amount of NGF protein decreased at 30 days and was not affected by virus infection. C) Expression of BDNF protein in adult rats was unaffected at 5 and 30 days post infection in all experimental groups. D) Expression of NGF protein in adult rats was unaffected at 5 and 30 days post infection in all experimental groups. Data are expressed as mean \pm SE ( $n=5-6$; ANOVA with Fisher LSD post-hoc analysis).

\section{mRNA Expression}

The expression of the neurotrophins NGF, BDNF and the receptors TrKb and p75 in lung homogenates as measured by mRNA levels (RT-PCR) did not reveal any significant changes in response to reovirus infection at 5 (Figure 19-A) and 30 days (Figure 19-B) in weanling rats. The expression of NGF and BDNF was significantly decreased in reovirus treated adult rats at 5 days post infection, while receptor expression was not affected (Figure 19-C). The changes in NGF and BDNF were no longer present at 30 days post infection and receptor expression continued to be unaffected (Figure 19-D). 

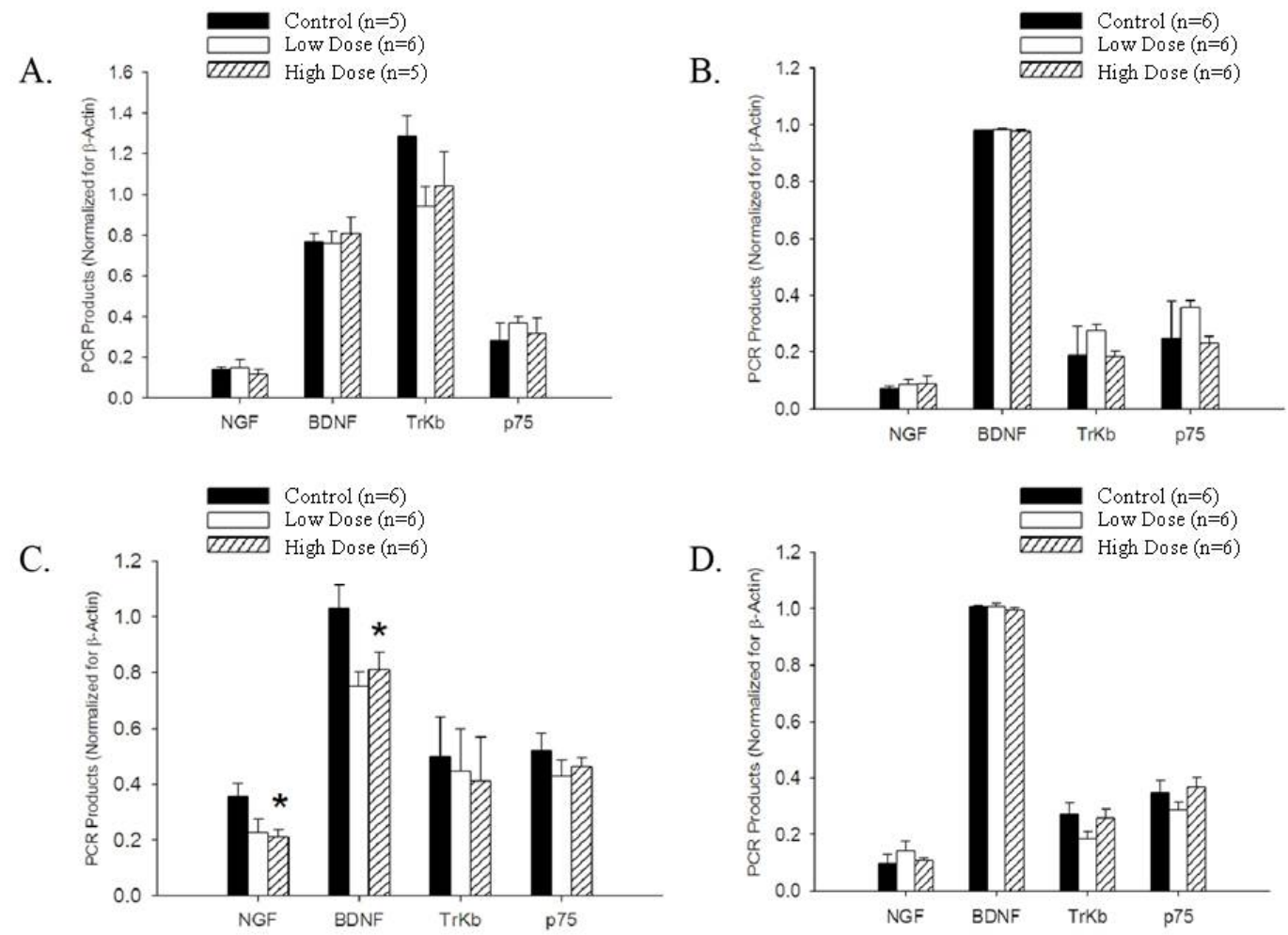

\section{Figure 19 mRNA Expression of Neurotrophins and their Receptors}

A) The semiquantitative RT-PCR analysis of NGF, BDNF, NGF high affinity receptor (TrKa), NGF low affinity receptor (p75), and BDNF receptor $(\mathrm{TrKb})$ mRNA products in lung tissues of weanling rats 5 and 30 days after inoculation with either low or high reovirus dose or virus-free vehicle did not reveal any significant changes. The amplified RT-PCR products were analyzed by electrophoresis on an ethidium bromide-stained $2 \%$ agarose gel. The gel was photographed and the intensity of the DNA bands was analyzed by computerized densitometry. Data are expressed as the ratio of the densitometric score measured for each target normalized by the $\beta$-actin control and are mean $+/-$ SE ( $n=5-6 ; p=n s$; ANOVA). B) The same analysis performed on adult rats lung homogenates 5 days after reovirus infection revealed a decreased expression of NGF and BDNF in infected rats compared to pathogen-free controls, whereas NGF high affinity receptor (TrKa) and low affinity receptor (p75) and BDNF receptor $(\mathrm{TrKb})$ were unaffected. These changes were lost 30 days post reovirus infection. The amplified RT-PCR products were analyzed by electrophoresis on an ethidium bromide-stained $2 \%$ agarose gel. The gel was photographed and the intensity of the DNA bands was analyzed by computerized densitometry. Data are expressed as the ratio of the densitometric score measured for each target normalized by the $\beta$-actin control and are mean $+/-\operatorname{SE}\left(n=5-6 ;{ }^{*} p<0.05\right.$; ANOVA with Fisher LSD post-hoc analysis).

\section{Caveolin-1 Protein Expression}

The effect of reovirus pulmonary infection on the expression of cav-1 was determined by western blot analysis. The expression of cav-1 protein at 5 days post infection was decreased in reovirus samples compared to controls (Figure 20-A). Statistical analysis determined that this decrease was not significant. At 30 days post infection Cav-1 protein expression was inconsistent, showing decreased and increased expression in response to reovirus infection 
(Figure 20-B). The magnitude of change in cav-1 expression was not dependent on the virus dose, but rather it was dependent upon the presence of fibrosis. Statistical analysis of samples exhibiting mild fibrosis as determined by a pathologist demonstrated significant decreases in cav-1 expression compared to controls (figure 20-D). In comparison, statistical analysis of samples exhibiting minimal to no signs of fibrosis in response to reovirus showed insignificant increased cav-1 expression compared to controls (Figure 20-E). At 60 days post infection, cav-1 expression appeared to decrease in response to virus infection when compared to controls (Figure 20-C), but was not significant. At 120 days cav-1 expression was not altered in comparison to controls (Figure 20-C). 
A.

5 Days Post Infectionn

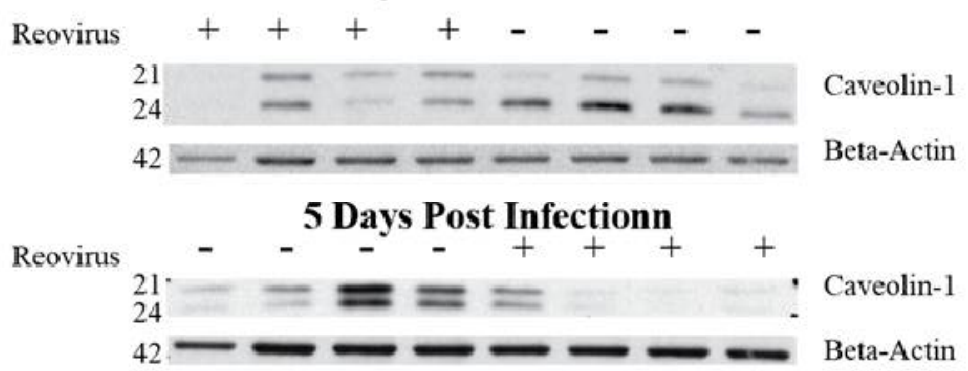

B.
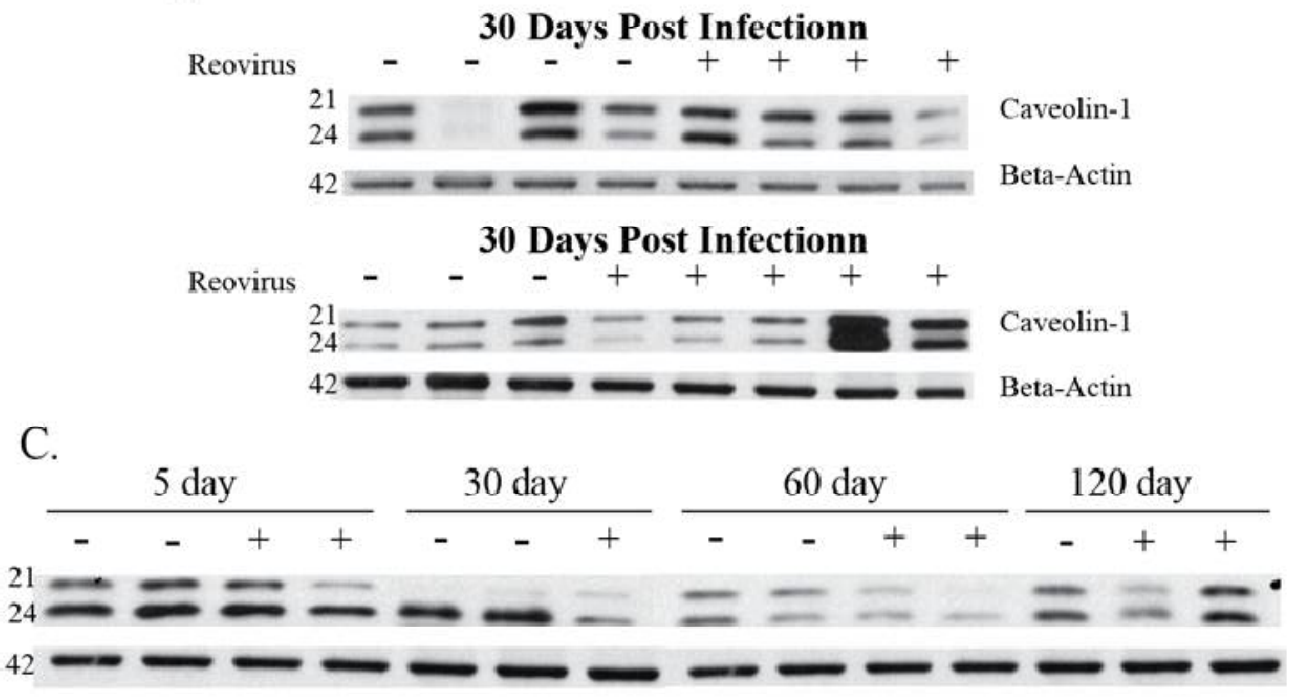

D.

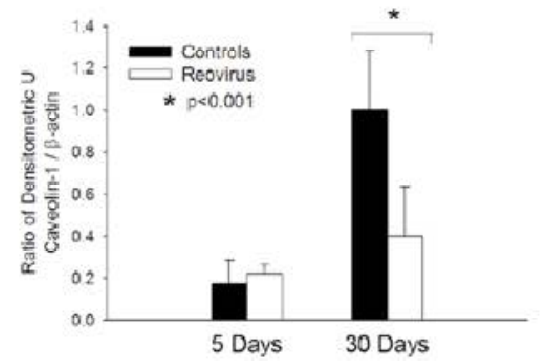

E.

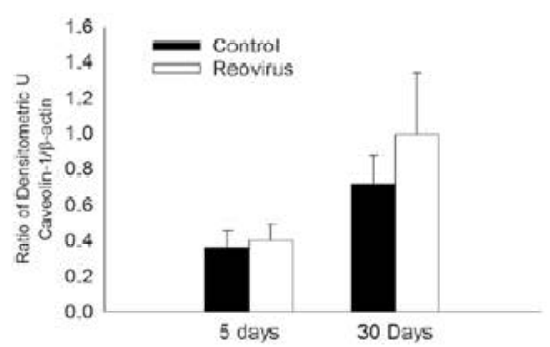

Figure 20 Analysis of Cav-1 Protein Expression post reovirus infection

A) Cav-1 protein expression at 5 days post infection was decreased in reovirus infected samples (indicated by + ) compared to controls (as indicated by -) as determined by immunoblot analysis of lung homogenates. B-actin serves as a loading control. B)Cav-1 expression at 30 days post infection was varied, with some samples showing decreased expression and othesr demonstrating increased expression in response to reovirus infection (indicated by + ) compared to controls (indicated by -). C) Cav-1 expression over the full time course, 5-120 days post infection as determined by immunoblot analysis using $\beta$-actin as a loading control. D) The expression of cav-1 in homogenates from rats lungs that were determined by hitological analysis to have significant fibrosis showed a significant decrease in cav-1 protein expression when compared to controls. Data are expressed as the ratio of the densitometric score measured for each target normalized by the $\beta$-actin control and are mean $+/-$ SE ( $n=6$; $* p<0.05$; ANOVA with Fisher LSD post-hoc analysis). E) Expression of cav-1 in samples from rats demonstrating less pronounced fibrosis in response to reovirus infection showed insignificant differencse in expression between reovirus infected samples and controls. The intensity of the protein bands was analyzed by computerized densitometry. Data are expressed as the ratio of the densitometric score measured for each target normalized by the $\beta$-actin control and are mean $+/-\mathrm{SE}\left(\mathrm{n}=6\right.$; ${ }^{*} \mathrm{p}<0.05$; ANOVA with Fisher LSD post-hoc analysis). 


\section{Concentration of PGs and LTs}

In order to determine the effect of reovirus infection on the synthesis of $\mathrm{PGE}_{2}$ and LTs in the airway we measured the concentration of each by enzyme immunoassay (EIA) at each time point (Figure 21-A). At 5 days post infection, the concentration of $\mathrm{PGE}_{2}$ was similar in the lungs of control and reovirus infected rats. $\mathrm{PGE}_{2}$ concentration diminished in response to reovirus infection compared to controls at 30 days. The difference in the median lung $\mathrm{PGE}_{2}$ concentrations between virus doses was minimal. At 60 days post infection the concentration of $\mathrm{PGE}_{2}$ in virus infected rats was diminished in response to both low and high dose infection when compared to controls, with high dose infected rats exhibiting the largest decrease in concentration. At 90 days post infection the amount of $\mathrm{PGE}_{2}$ was increased in reovirus infected rats, with high dose infection showing the greatest increase. At 120 days post infection $\mathrm{PGE}_{2}$ was decreased in control and low dose reovirus infected rats. In comparison rats infected with high dose demonstrated greater $\mathrm{PGE}_{2}$ levels. Statistical analysis revealed that all changes in concentration were not significant.

Reovirus infected rats' demonstrated increased amounts of LTs in the airway compared to controls at 5 days post infection (Figure 21-B). Changes in the concentration of airway LTs were no longer present at 30 days. LT concentration continued to be similar between the three experimental groups at 60 days post infection. At 90 days post infection the amount of LTs in the airway increased in high dose infected animals compared to both control and low dose. Increased LT synthesis in both low and high dose reovirus infected rats continued at 120 days post infection when compared to controls. Though there were apparent differences between experimental groups, there were no significant differences between groups. 

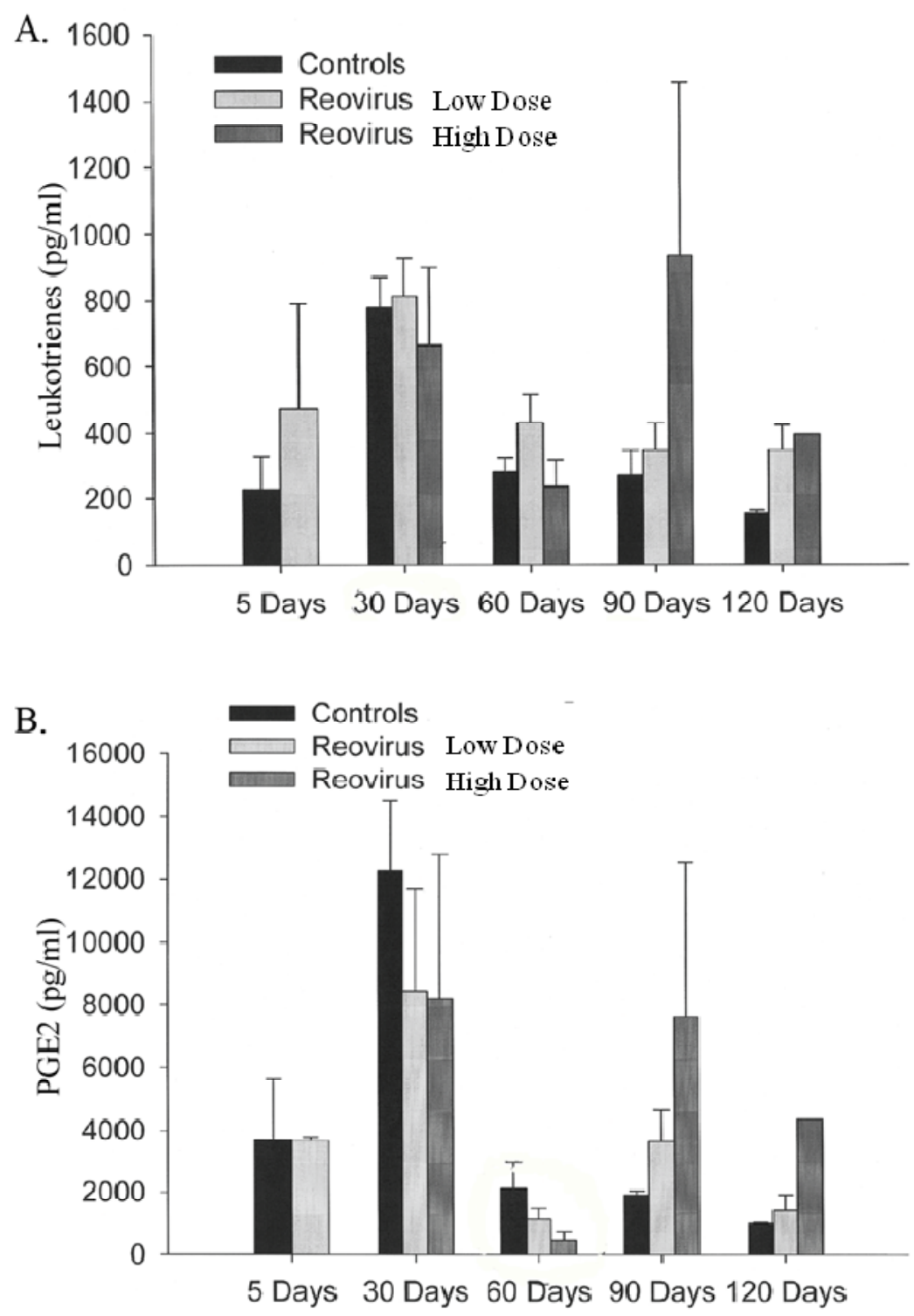

Figure 21 Expression of the hormones PGE2 and LT in response to reovirus infection

A) Concentration of LTs in lung homogenates from control and reovirus infected rats at 5, 30, 60, 90 and 120 days post infection were analyzed by using a specific enzyme immunoassay technique. Changes in LT concentration were not significant between experimental groups. Data represents the mean +/- SE ( $n=5-6$; ANOVA with Fisher LSD post-hoc analysis). B) Expression of $\mathrm{PGE}_{2}$ in lung homogenates from control and reovirus infected rats at 5, 30, 60, 90 and 120 days post infection were analyzed by using a specific enzyme immunoassay technique. Changes in $\mathrm{PGE}_{2}$ expression were not significant between experimental groups. Data represents the mean +/- SE ( $n=5-6$; ANOVA with Fisher LSD post-hoc analysis). 


\section{CHAPTER 5}

\section{Discussion}

\section{Reovirus and Neurogenic Inflammation}

Viral respiratory infections early in life have a significant impact on the developing airway resulting in pathogenic long term effects such as asthma and fibrosis. Viral induced dysregulation of the airway's neuroimmune interactions by RSV have been shown to result in airway hyper reactivity from potentiated neurogenic inflammation in response to augmented NGF and trkA expression. In this study, we used another common early life pathogen, reovirus, in a rat model of airway inflammation to reveal its effects on the airway's physiological responses. Our data show that acute reovirus LRTI in adult (12 week-old) and weanling (2 week-old) rats results in short term potentiation of neurogenic-mediated inflammation that coincides with acute inflammatory responses. This was indicated by the increased microvascular permeability following stimulation of capsaicin-sensitive sensory nerves 5 days after virus inoculation. This effect is lost in weanling rats 30 days after infection, but is still present in adult rats treated with high dose reovirus. The intrapulmonary and extra pulmonary airway responses were differentially affected in the two age groups as demonstrated by the differences in Evansblue labeled albumin extravasation in response to capsaicin. In weanling rats, the potentiation of capsaicin-induced neurogenic inflammation was significant in the lung or intrapulmonary airways, but not in the extra pulmonary airways of infected animals at only the 5 day time point. In adult rats, this effect was significant in both the extra pulmonary and intrapulmonary airways and was present at both 5 and 30 days. The age-related differential sensitivity of intra- and extra pulmonary airways to viral infection is consistent with previous observations in rats infected with RSV [295, 301] and reflects the developmental modification in the anatomic distribution of 
sensory nerve fibers across the respiratory tract [298, 302]. However, unlike RSV, reovirus did not result in potentiation of neurogenic inflammation at 30 days in weanling rats. Nonetheless, our data are the first to document such a physiological response in the airways of rats following lower respiratory tract infection with reovirus, suggesting that other viral pathogens can cause neurogenic inflammation.

Potentiation of neurogenic inflammation was observed in adult rats that received high dose virus leading to the hypothesis that viral dose may be a critical factor in potentiating neurogenic inflammation. More specifically, increasing severity of infection in response to higher doses of reovirus may directly correlate with increases in remodeling to airway innervations. In order to determine if higher doses of reovirus could induce potentiated neurogenic inflammation further studies infecting rats with a titer of reovirus greater then $2.5 \times 10^{6} \mathrm{PFU} / \mu 1$ would have to be done. This may be difficult in weanling rats since both high and low dose reovirus resulted in significant weight loss indicative of a severe infection, but may be possible in adult rats. Measuring the Evans-blue labeled albumin extravasation in response to a higher dose of reovirus could indicate whether or not potentiation of neurogenic inflammation is dose dependent. Furthermore, infection severity as determined by histolopathologic analysis and measurement of body weight in response to a higher dose of reovirus could provide a correlation between severity and changes in airway innervations.

Another important component that could be involved in reovirus induced changes to neurogenic inflammatory responses is neutral endopeptidase (NEP). NEP is a membrane bound metalloproteinase that exists at the surface of AECs, SMCs, sub mucosal gland cells and fibroblasts. The key role of NEP is to limit the actions of the sensory peptides known as tachykinins, such as SP, that provoke neurogenic inflammation. It preferentially cleaves SP into 
inactive fragments that lack the carboxyl terminal region that is necessary for the binding to NK1. Therefore, NEP activity is responsible for preventing SP induced exaggerated neurogenic inflammation. Studies have shown viral infection, and exposure to air pollutants and allergens are able to reduce NEP activity [303]. We speculate that reovirus infection destabilizes the integrity of the airway epithelium by either directly affecting epithelial cells or indirectly by inducing the production of detrimental inflammatory factors resulting in reduced NEP activity. As a result of reduced NEP, SP is able to induce exaggerated neurogenic inflammation during acute infection (5 days). At the cessation of acute inflammation (30 days) the detrimental factors are no longer present and the integrity of the airway epithelium returns as does the normal level of NEP activity. Reinstated NEP activity removes any excess SP, preventing an exaggerated neurogenic inflammatory response. The severity of infection is important in controlling the amount of damage or injury to the epithelium and could be the determining factor in whether or not changes in NEP can be returned to normal or become a chronic condition. Higher doses of reovirus could produce a more severe LRTI characterized by more significant injury to the airway epithelium, resulting in chronic reduced NEP activity. Persistent decreased NEP explains the potentiation of neurogenic inflammation in adult rats 30 days post infection with high dose reovirus. Therefore, higher doses of virus produce more severe LRTIs that can permanently switch the neurogenic airway responses from protective to detrimental, resulting in perpetuated and augmented airway inflammation.

Previous work has shown that the potentiation of neurogenic inflammation observed during acute infection with RSV is associated with up regulation of the neurotrophin NGF and its high and low affinity receptors (TrKa, and p75) which, in turn, controls the expression of the gene encoding the peptide neurotransmitter SP resulting in the increased release of this 
neuropeptide from airway sensory nerves [292]. It also induces increased expression of the SP high affinity receptor NK-1 in the airway, further contributing to exacerbating the neurogenic inflammatory response. In our study, we did not observe any significant changes in the expression of the neurotrophins NGF and BDNF as well as their receptors in weanling rats following reovirus infection, but we did observe a significant decrease in NGF and BDNF expression in adult rats at 5 days but not 30 days. Our findings, therefore, suggest a different mechanism that is not associated with NGF-driven up regulation of SP and NK-1 expression is responsible for reovirus induced neurogenic inflammation. Therefore, our results demonstrate that reovirus produces augmented neurogenic inflammation that is mediated by SP and NK-1 but the mechanism responsible for the change is not clear.

SP is one of the most effective and versatile inflammatory mediators known. It greatly increases endothelial permeability and blood flow in post capillary venules, causing airway edema; stimulates the proliferation and activation of $\mathrm{T}$ and $\mathrm{B}$ lymphocytes; attracts leukocytes to the vascular endothelium; causes degranulation of MCs with release of other mediators of inflammation; and primes and activates monocytes and macrophages to release a variety of cytokines such as TNF- $\alpha$ and IL-6 [304-310]. Receptors for SP have been identified in all these and various other cell types in humans. Although SP has been described as a peptide of neuronal origin, research has shown it is also produced by inflammatory cells such as macrophages, DCs and lymphocytes, during inflammation [308, 310-311]. In addition, augmented neurogenic inflammation that is mediated by SP was mainly associated with acute infection and inflammation, indicating that components of the inflammatory response to reovirus may act as regulatory mediators. Therefore, one possible hypothesis is that reovirus infection induces increased SP production and NK-1 expression by inflammatory cells resulting in augmented 
acute neurogenic inflammation. These changes are not potentiated because of the decreased presence of inflammatory cells at the chronic stage (30 days) of infection. Our results indicate that the acute inflammatory response to reovirus infection results in increased infiltration of the alveolar interstitium by inflammatory cells, specifically macrophages, PMNs and lymphocytes. The infiltrating inflammatory cells present at 5 days could serve as the source of increased SP that exacerbates the inflammatory processes and enhances neurogenic inflammation. At 30 days the population and number of inflammatory cells present in the alveolar interstitium is altered, with a decreased number of infiltrating cells that consist of lymphocytes and few to no acute inflammatory cells resulting in a less robust SP response to capsaicin and no potentiation of neurogenic inflammation at 30 days. However, in the present study, the capacities of infiltrating inflammatory cells in reovirus-induced airway responses were not investigated.

\section{Reovirus and Inflammation}

The acute changes in vascular permeability coincided with increased inflammatory cell infiltration into the respiratory bronchioles and alveolar spaces of rat lungs 5 days post infection. The peribronchiolar and perivascular accumulation of inflammatory cells such as lymphocytes and neutrophils was consistent with previous observations in rodents [7]. At 30 days post infection, chronic inflammatory cells were observed in the bronchiole spaces and the interstitium of virus infected rats. The presence of neutrophils and faint cytoplasmic vacuoles and basophilic foci associated with viral inclusion was observed in and specific to high dose reovirus infection. This indicated that the virus dose may have an effect on the long term inflammatory responses. However, there were no observed histological differences between the two virus doses at 60 days post infection. Both doses resulted in continued chronic inflammation around respiratory 
bronchioles along with a few foamy macrophages in the lumen of terminal respiratory bronchioles.

\section{Reovirus and Fibrosis}

The early signs of fibrosis development were observed in reovirus weanling rats 30 days post reovirus infection as demonstrated by increased collagen accumulation and increased fibroblast localization to respiratory bronchioles and alveolar spaces. Reovirus infection resulted in focal, mild fibrosis. Fibrotic regions demonstrated decreased vascular perfusion in conjunction with increases in epithelial cells and fibroblasts. These regions were located in the interstitial spaces and were associated with increases in collagen and number of fibroblasts being found, specifically at terminal respiratory bronchioles and alveolar spaces, suggesting interstitial fibrosis. These results are unique to reovirus and have not been observed in response to other early life pathogens such as RSV. This demonstrates that early life reovirus infection may be a potential risk or initiating factor for remodeling of the airway.

Our preliminary results demonstrated that cav-1 may be a relevant influential factor in the pathogenesis of reovirus induced airway remodeling. The expression of cav-1 protein was not consistent, but varied between rats and time points, providing contradictory results. Samples from rats that demonstrated significant fibrosis at 30 days exhibited decreased cav-1 protein expression, while samples from rats demonstrating less pronounced fibrosis showed cav-1 expression that was either equivalent to or greater than controls. This correlates with previous in vitro and in vivo studies of bleomycin induced fibrosis as well as observations of IPF lung samples where cav-1 expression is decreased, indicating cav-1 is anti-fibrogenic. Previous studies did not look at time points past 30 days, but we observed at 60 days post infection, cav-1 expression appeared to decrease in response to virus infection when compared to controls, but 
this observation is based on a limited sample size of 2 . Such a limited sample size does not depict a realistic or significant collection for analysis. Increasing the number of samples would provide a more comprehensive representation of the changes induced by reovirus infection and would allow us to determine whether the changes observed could be statistically significant. Interestingly, at 120 days cav-1 expression was not altered in comparison to controls, but again we had a very small sample size of 1 control and 2 reovirus infected rats. Therefore, repeating this experiment would provide a larger samples size that would allow for more useful and informative analysis. However, this experiment did provide the important observation that cav-1 expression at 30 days is significantly decreased in fibrotic samples. We speculate that reovirus LRTI decreases airway cav-1 expression, reversing the inhibition of profibrogenic processes resulting in the development of fibrosis. It has been well documented in previous studies that TGF- $\beta$ signaling can serve as a primary stimulus for fibrogenic processes such as fibroblast activation and synthesis of ECM [46-48]. More importantly, it has also been determined that the cav-1 is a key regulator in TGF- $\beta$ profibrogenic signaling (Figure 6), inhibiting the activation of key signaling cascades and therefore preventing fibrogenic processes [102]. Based on this, we suppose a mechanism for reovirus induced fibrosis where decreased cav-1 expression results in increased TGF- $\beta$ profibrogenic signaling and the development of fibrosis. Further investigation is needed in order to determine if TGF- $\beta$ is influential in the pathogenesis of reovirus induced fibrosis. Primarily, it would be necessary to look at TGF- $\beta$ expression at both the protein and transcriptional level to determine if its activity is altered by reovirus LRTI. Nonetheless, our results demonstrate that cav-1 expression is altered post reovirus infection and could be relevant in the mechanisms responsible for reovirus induced fibrosis. 
Eicosanoids have been implicated as key mediators in the development of fibropriliferation in other fibrotic diseases. Our results demonstrate that neither $\mathrm{PGE}_{2}$ nor LTs are involved in the pathogenesis of reovirus induced fibrosis, as implicated by no changes in the synthesis of either hormone in response to reovirus LRTI. The method used for the quantification of both $\mathrm{PGE}_{2}$ and the LTs was imprecise, as demonstrated by the large significant error between samples. Increasing the number of samples would allow for more precise calculations of $\mathrm{PGE}_{2}$ and LT sample concentration. Further investigation using other methods of analysis could demonstrate whether or not PGs and/or LTs are influential in reovirus induced fibrosis. One means of investigating this would be to observe the activity of the enzymes involved in their synthesis. Analysis of COX-2 transcription would indicate changes in the level of $\mathrm{PGE}_{2}$ synthesis, based on the fact that COX-1 is constitutively expressed and COX-2 expression is only present during periods of increased PG synthesis. Therefore, changes in COX-2 transcription would be indicative of increased $\mathrm{PGE}_{2}$. Analysis of 5-LO transcription would provide similar information about the level of LT synthesis. A second means of investigating the involvement of both hormones would be to inhibit their synthesis or receptor binding and observe if changes in fibrotic development occur. Nonetheless, we concluded from our data that eicosanoids do not play an influential role in the pathogenesis of reovirus induced fibrosis.

\section{Conclusion}

In conclusion, our data show that reovirus, a commonly occurring airway pathogen, causes acute neurogenic-mediated airway inflammation which, unlike RSV, is not associated with the NGF-mediated disruption of neurosensory airway innervations. In weanling rats these physiological responses are associated with the development of mild pulmonary fibrosis and, 
therefore indicate the potential for long-lasting sequelae. Regulation of reovirus induced fibrosis may be influenced by cav-1 expression, but does not involve the eicosanoids. Additional studies are necessary to investigate the mechanisms involved in the reovirus-induced airway responses and whether they represent potential risk factors for the development of airway diseases. Taken together this reports that early life reovirus LRTI has both acute and long term effects on airway physiology that results in acute augmentation of airway neurogenic inflammation and subsequent development of fibrosis that is mediated by unique mechanisms specific to reovirus. Our study suggests that the mediators involved may provide novel insight into the processes involved in airway remodeling disease and provide possible therapeutic answers. 


\section{REFERENCES}

1. Piedimonte, G., Origins of reactive airways disease in early life: do viral infections play a role? Acta Paediatr Suppl, 2002. 91(437): p. 6-11.

2. Cebra, J.J., et al., Reoviruses as probes of the gut mucosal T cell population. Immunol Invest, 1989. 18(1-4): p. 545-58.

3. Tyler, K., in Fields Virology, H.P. Knipe DM, Griffin DE, Lamb RA, Martin MA, Roizman B, Straus SE., Editor. 2001, Lippincott Williams \& Wilkins: Philidelphia. p. 1729-1745.

4. Chappell, J.D., R.; Mertens, PPC.; Dermody, TS., in Virus Taxonomy: Eighth Report of the International Committee on Taxonomy of Viruses, M.M. Fauquet CM, Maniloff J, Desselberger U, Ball LA., Editor. 2005, Elsevier Academic: San Diego. p. 455-465.

5. Sabin, A.B., Reoviruses. A new group of respiratory and enteric viruses formerly classified as ECHO type 10 is described. Science, 1959. 130: p. 1387-9.

6. Jackson, G.G. and R.L. Muldoon, Viruses causing common respiratory infections in man. J Infect Dis, 1973. 127(3): p. 328-55.

7. Morin, M.J., A. Warner, and B.N. Fields, Reovirus infection in rat lungs as a model to study the pathogenesis of viral pneumonia. J Virol, 1996. 70(1): p. 541-8.

8. Zalan, E., W.D. Leers, and N.A. Labzoffsky, Occurrence of reovirus infection in Ontario. Can Med Assoc J, 1962. 87: p. 714-5.

9. Watanabe, Y. and A.F. Graham, Structural units of reovirus ribonucleic acid and their possible functional significance. J Virol, 1967. 1(4): p. 665-77.

10. Weiner, H.L., et al., Molecular basis of reovirus virulence: role of the S1 gene. Proc Natl Acad Sci U S A, 1977. 74(12): p. 5744-8.

11. Tarlow, O., J.G. McCorquodale, and M.A. McCrae, Molecular cloning and sequencing of the gene (M2) encoding the major virion structural protein (mu 1-mu 1C) of serotypes 1 and 3 of mammalian reovirus. Virology, 1988. 164(1): p. 141-6.

12. Joklik, W.K., The structure and function of the reovirus genome. Ann N Y Acad Sci, 1980. 354: p. 107-24.

13. Chantal Hulo, P.M., Edouard de Castro, Philippe Le Mercier. ViralZone. Orthoreovirus 2010 [cited 2010 8/2010]; Available from: http://www.expasy.ch/viralzone/all by protein/105.html.

14. Silverstein, S.C., J.K. Christman, and G. Acs, The reovirus replicative cycle. Annu Rev Biochem, 1976. 45: p. 375-408.

15. Shmulevitz, M., P. Marcato, and P.W. Lee, Unshackling the links between reovirus oncolysis, Ras signaling, translational control and cancer. Oncogene, 2005. 24(52): p. 7720-8.

16. Weiner, H.L., et al., Identification of the gene coding for the hemagglutinin of reovirus. Virology, 1978. 86(2): p. 581-4.

17. Leers, W.D. and K.R. Rozee, A survey of reovirus antibodies in sera of urban children. Can Med Assoc J, 1966. 94(20): p. 1040-2.

18. El-Rai, F.M. and A.S. Evans, Reovirus Infections in Children and Young Adults. Arch Environ Health, 1963. 7: p. 700-4.

19. Lerner, A.M., et al., Infections with reoviruses. N Engl J Med, 1962. 267: p. 947-52.

20. Tai, J.H., et al., Prevalence of reovirus-specific antibodies in young children in Nashville, Tennessee. J Infect Dis, 2005. 191(8): p. 1221-4. 
21. Tillotson, J.R. and A.M. Lerner, Reovirus type 3 associated with fatal pneumonia. N Engl J Med, 1967. 276(19): p. 1060-3.

22. Liotet, S., M.J. Wattiaux, and Y. Morin, Gingival impression: a new biological test for xerostomia in diagnosis of Sjogren's syndrome. Adv Exp Med Biol, 1994. 350: p. 655-9.

23. Thompson, A.H., et al., Respiratory-mucosal lymphocyte populations induced by reovirus serotype 1 infection. Cell Immunol, 1996. 169(2): p. 278-87.

24. Periwal, S.B. and J.J. Cebra, Respiratory mucosal immunization with reovirus serotype 1/L stimulates virus-specific humoral and cellular immune responses, including doublepositive (CD4(+)/CD8(+)) T cells. J Virol, 1999. 73(9): p. 7633-40.

25. Farone, A.L., et al., Serotype-dependent induction of pulmonary neutrophilia and inflammatory cytokine gene expression by reovirus. J Virol, 1996. 70(10): p. 7079-84.

26. Virgin, H.W., T.S. Dermody, and K.L. Tyler, Cellular and humoral immunity to reovirus infection. Curr Top Microbiol Immunol, 1998. 233(Pt 2): p. 147-61.

27. Mathers, A.R. and C.F. Cuff, Role of interleukin-4 (IL-4) and IL-10 in serum immunoglobulin $G$ antibody responses following mucosal or systemic reovirus infection. J Virol, 2004. 78(7): p. 3352-60.

28. Epler, G.R., et al., Bronchiolitis obliterans organizing pneumonia. N Eng1 J Med, 1985. 312(3): p. 152-8.

29. Colby, T.V., Bronchiolitis. Pathologic considerations. Am J Clin Pathol, 1998. 109(1): p. 101-9.

30. Gosink, B.B., P.J. Friedman, and A.A. Liebow, Bronchiolitis obliterans. Roentgenologicpathologic correlation. Am J Roentgenol Radium Ther Nucl Med, 1973. 117(4): p. 81632.

31. Dale, R.C., et al., Brochiolitis obliterans; long-term follow-up. N Y State J Med, 1977. 77(9): p. 1485-8.

32. Epler, G.R., Constrictive bronchiolitis obliterans: the fibrotic airway disorder. Expert Rev Respir Med, 2007. 1(1): p. 139-47.

33. Epler, G.R., Bronchiolitis obliterans organizing pneumonia. Semin Respir Infect, 1995. 10(2): p. 65-77.

34. Epler, G.R., Bronchiolitis obliterans organizing pneumonia: definition and clinical features. Chest, 1992. 102(1 Suppl): p. 2S-6S.

35. Epler, G.R. and T.V. Colby, The spectrum of bronchiolitis obliterans. Chest, 1983. 83(2): p. 161-2.

36. Izumi, T., [Bronchiolitis obliterans organizing pneumonia (BOOP)]. Ryoikibetsu Shokogun Shirizu, 1994(3): p. 585-7.

37. Nizami, I.Y., et al., Idiopathic bronchiolitis obliterans with organizing pneumonia. An acute and life-threatening syndrome. Chest, 1995. 108(1): p. 271-7.

38. Bellum, S.C., et al., Respiratory reovirus $1 / L$ induction of intraluminal fibrosis. A model for the study of bronchiolitis obliterans organizing pneumonia. Am J Pathol, 1997. 150(6): p. 2243-54.

39. Raghu, G., et al., Incidence and prevalence of idiopathic pulmonary fibrosis. Am J Respir Crit Care Med, 2006. 174(7): p. 810-6.

40. Desmouliere, A., Factors influencing myofibroblast differentiation during wound healing and fibrosis. Cell Biol Int, 1995. 19(5): p. 471-6.

41. Strieter, R.M., What differentiates normal lung repair and fibrosis? Inflammation, resolution of repair, and fibrosis. Proc Am Thorac Soc, 2008. 5(3): p. 305-10. 
42. Hardie, W.D., S.W. Glasser, and J.S. Hagood, Emerging concepts in the pathogenesis of lung fibrosis. Am J Pathol, 2009. 175(1): p. 3-16.

43. Crosby, L.M. and C.M. Waters, Epithelial repair mechanisms in the lung. Am J Physiol Lung Cell Mol Physiol, 2010. 298(6): p. L715-31.

44. Kasper, M., et al., Early signs of lung fibrosis after in vitro treatment of rat lung slices with CdCl2 and TGF-betal. Histochem Cell Biol, 2004. 121(2): p. 131-40.

45. Sato, M., et al., Lysophosphatidic acid inhibits TGF-beta-mediated stimulation of type I collagen $m R N A$ stability via an ERK-dependent pathway in dermal fibroblasts. Matrix Biol, 2004. 23(6): p. 353-61.

46. Gauldie, J., et al., TGF-beta, Smad3 and the process of progressive fibrosis. Biochem Soc Trans, 2007. 35(Pt 4): p. 661-4.

47. Secker, G.A., et al., TGFbeta stimulated re-epithelialisation is regulated by CTGF and Ras/MEK/ERK signalling. Exp Cell Res, 2007.

48. Willis, B.C. and Z. Borok, TGF-beta-induced EMT: mechanisms and implications for fibrotic lung disease. Am J Physiol Lung Cell Mol Physiol, 2007. 293(3): p. L525-34.

49. Desmouliere, A., et al., Transforming growth factor-beta 1 induces alpha-smooth muscle actin expression in granulation tissue myofibroblasts and in quiescent and growing cultured fibroblasts. J Cell Biol, 1993. 122(1): p. 103-11.

50. Mitchell, J., et al., Alpha-smooth muscle actin in parenchymal cells of bleomycin-injured rat lung. Lab Invest, 1989. 60(5): p. 643-50.

51. Zhang, H.Y., et al., Lung fibroblast alpha-smooth muscle actin expression and contractile phenotype in bleomycin-induced pulmonary fibrosis. Am J Pathol, 1996. 148(2): p. 527-37.

52. Williams, T.M. and M.P. Lisanti, The caveolin proteins. Genome Biol, 2004. 5(3): p. 214.

53. Schlegel, A., et al., Crowded little caves: structure and function of caveolae. Cell Signal, 1998. 10(7): p. 457-63.

54. Palade, G., Fine Structure of Blood Capillaries. J Appl Physiol, 1953. 24: p. 1424-1436.

55. Yamada, E., The fine structure of the gall bladder epithelium of the mouse. J Biophys Biochem Cytol, 1955. 1(5): p. 445-58.

56. Anderson, R.G., Caveolae: where incoming and outgoing messengers meet. Proc Natl Acad Sci U S A, 1993. 90(23): p. 10909-13.

57. Harder, T. and K. Simons, Caveolae, DIGs, and the dynamics of sphingolipid-cholesterol microdomains. Curr Opin Cell Biol, 1997. 9(4): p. 534-42.

58. Michel, C.C., Capillaries, caveolae, calcium and cyclic nucleotides: a new look at microvascular permeability. J Mol Cell Cardiol, 1998. 30(12): p. 2541-6.

59. Liu, J. and J.E. Schnitzer, Analysis of lipids in caveolae. Methods Mol Biol, 1999. 116: p. 61-72.

60. Parton, R.G., Caveolae and caveolins. Curr Opin Cell Biol, 1996. 8(4): p. 542-8.

61. Gumbleton, M., A.G. Abulrob, and L. Campbell, Caveolae: an alternative membrane transport compartment. Pharm Res, 2000. 17(9): p. 1035-48.

62. Gratton, J.P., P. Bernatchez, and W.C. Sessa, Caveolae and caveolins in the cardiovascular system. Circ Res, 2004. 94(11): p. 1408-17.

63. Silva, W., et al., Caveolae a new subcellular transport organelle. Bol Asoc Med P R, 1998. 90(1-3): p. 30-3. 
64. Schnitzer, J.E., Caveolae: from basic trafficking mechanisms to targeting transcytosis for tissue-specific drug and gene delivery in vivo. Adv Drug Deliv Rev, 2001. 49(3): p. 26580 .

65. Kirkham, M. and R.G. Parton, Clathrin-independent endocytosis: new insights into caveolae and non-caveolar lipid raft carriers. Biochim Biophys Acta, 2005. 1745(3): p. 273-86.

66. van Deurs, B., et al., Are caveolae involved in clathrin-independent endocytosis? Trends Cell Biol, 1993. 3(8): p. 249-51.

67. Anderson, R.G., Potocytosis of small molecules and ions by caveolae. Trends Cell Biol, 1993. 3(3): p. 69-72.

68. Anderson, R.G., et al., Potocytosis: sequestration and transport of small molecules by caveolae. Science, 1992. 255(5043): p. 410-1.

69. Pelkmans, L. and A. Helenius, Endocytosis via caveolae. Traffic, 2002. 3(5): p. 311-20.

70. Smart, E.J., et al., Caveolins, liquid-ordered domains, and signal transduction. Mol Cell Biol, 1999. 19(11): p. 7289-304.

71. Liu, J., et al., Organized endothelial cell surface signal transduction in caveolae distinct from glycosylphosphatidylinositol-anchored protein microdomains. J Biol Chem, 1997. 272(11): p. 7211-22.

72. Patel, H.H., F. Murray, and P.A. Insel, G-protein-coupled receptor-signaling components in membrane raft and caveolae microdomains. Handb Exp Pharmacol, 2008(186): p. 167-84.

73. Chen, C.Y. and J.R. King, Modelling the effect of caveolae on G-protein activation. Bull Math Biol, 2006. 68(4): p. 863-88.

74. Insel, P.A., et al., Compartmentation of G-protein-coupled receptors and their signalling components in lipid rafts and caveolae. Biochem Soc Trans, 2005. 33(Pt 5): p. 1131-4.

75. Chini, B. and M. Parenti, G-protein coupled receptors in lipid rafts and caveolae: how, when and why do they go there? J Mol Endocrinol, 2004. 32(2): p. 325-38.

76. Lohse, M.J., et al., Regulators of G-protein-mediated signalling. Biochem Soc Trans, 1996. 24(4): p. 975-80.

77. Schwencke, C., et al., Caveolae and caveolin in transmembrane signaling: Implications for human disease. Cardiovasc Res, 2006. 70(1): p. 42-9.

78. Hnasko, R. and M.P. Lisanti, The biology of caveolae: lessons from caveolin knockout mice and implications for human disease. Mol Interv, 2003. 3(8): p. 445-64.

79. Razani, B. and M.P. Lisanti, Caveolin-deficient mice: insights into caveolar function human disease. J Clin Invest, 2001. 108(11): p. 1553-61.

80. Campbell, L., M. Gumbleton, and K. Ritchie, Caveolae and the caveolins in human disease. Adv Drug Deliv Rev, 2001. 49(3): p. 325-35.

81. Schlegel, A., R.G. Pestell, and M.P. Lisanti, Caveolins in cholesterol trafficking and signal transduction: implications for human disease. Front Biosci, 2000. 5: p. D929-37.

82. Kurzchalia, T.V., P. Dupree, and S. Monier, VIP21-Caveolin, a protein of the trans-Golgi network and caveolae. FEBS Lett, 1994. 346(1): p. 88-91.

83. Kurzchalia, T.V. and R.G. Parton, Membrane microdomains and caveolae. Curr Opin Cell Biol, 1999. 11(4): p. 424-31.

84. Rothberg KG, H.J., Donzell WC, Ying YS, Glenney JR, Anderson RG., Caveolin, a protein componenet of caveolae membrane coats, in Cell 1992. p. 673-682. 
85. Fra, A.M., et al., De novo formation of caveolae in lymphocytes by expression of VIP21caveolin. Proc Natl Acad Sci U S A, 1995. 92(19): p. 8655-9.

86. Tang Z, S.P., Okamoto T, Song K, Chu C, Kohtz DS, Nishimoto I, Lodish HF, Lisanti MP, Molecular cloning of caveolin-3, a novel member of the caveolin gene family expressed predominantly in muscle. J Biol Chem, 1996. 271(4): p. 2255-2261.

87. Mora, R., et al., Caveolin-2 localizes to the golgi complex but redistributes to plasma membrane, caveolae, and rafts when co-expressed with caveolin-1. J Biol Chem, 1999. 274(36): p. 25708-17.

88. Schlegel, A., et al., A role for the caveolin scaffolding domain in mediating the membrane attachment of caveolin-1. The caveolin scaffolding domain is both necessary and sufficient for membrane binding in vitro. J Biol Chem, 1999. 274(32): p. 22660-7.

89. Schlegel, A. and M.P. Lisanti, A molecular dissection of caveolin-1 membrane attachment and oligomerization. Two separate regions of the caveolin-1 C-terminal domain mediate membrane binding and oligomer/oligomer interactions in vivo. J Biol Chem, 2000. 275(28): p. 21605-17.

90. Woodman, S.E., et al., Mutational analysis identifies a short atypical membrane attachment sequence (KYWFYR) within caveolin-1. Biochemistry, 2002. 41(11): p. 37905.

91. Galbiati, F., et al., The dually acylated NH2-terminal domain of gilalpha is sufficient to target a green fluorescent protein reporter to caveolin-enriched plasma membrane domains. Palmitoylation of caveolin-1 is required for the recognition of dually acylated g-protein alpha subunits in vivo. J Biol Chem, 1999. 274(9): p. 5843-50.

92. Song, K.S., et al., Mutational analysis of the properties of caveolin-1. A novel role for the $C$-terminal domain in mediating homo-typic caveolin-caveolin interactions. J Biol Chem, 1997. 272(7): p. 4398-403.

93. Razani, B. and M.P. Lisanti, Two distinct caveolin-1 domains mediate the functional interaction of caveolin-1 with protein kinase A. Am J Physiol Cell Physiol, 2001. 281(4): p. C1241-50.

94. Abulrob, A., et al., Interactions of EGFR and caveolin-1 in human glioblastoma cells: evidence that tyrosine phosphorylation regulates EGFR association with caveolae. Oncogene, 2004. 23(41): p. 6967-79.

95. Lu, Z., et al., Downregulation of caveolin-1 function by EGF leads to the loss of Ecadherin, increased transcriptional activity of beta-catenin, and enhanced tumor cell invasion. Cancer Cell, 2003. 4(6): p. 499-515.

96. Lee, H., et al., Constitutive and growth factor-regulated phosphorylation of caveolin-1 occurs at the same site (Tyr-14) in vivo: identification of a c-Src/Cav-1/Grb7 signaling cassette. Mol Endocrinol, 2000. 14(11): p. 1750-75.

97. Kim, Y.N., et al., Epidermal growth factor-stimulated tyrosine phosphorylation of caveolin-1. Enhanced caveolin-1 tyrosine phosphorylation following aberrant epidermal growth factor receptor status. J Biol Chem, 2000. 275(11): p. 7481-91.

98. Peterson, T.E., et al., Caveolin-1 can regulate vascular smooth muscle cell fate by switching platelet-derived growth factor signaling from a proliferative to an apoptotic pathway. Arterioscler Thromb Vasc Biol, 2003. 23(9): p. 1521-7.

99. Wary, K.K., et al., A requirement for caveolin-1 and associated kinase Fyn in integrin signaling and anchorage-dependent cell growth. Cell, 1998. 94(5): p. 625-34. 
100. Sedding, D.G., et al., Caveolin-1 facilitates mechanosensitive protein kinase B (Akt) signaling in vitro and in vivo. Circ Res, 2005. 96(6): p. 635-42.

101. Li, S., J. Couet, and M.P. Lisanti, Src tyrosine kinases, Galpha subunits, and H-Ras share a common membrane-anchored scaffolding protein, caveolin. Caveolin binding negatively regulates the auto-activation of Src tyrosine kinases. J Biol Chem, 1996. 271(46): p. 29182-90.

102. Razani, B., et al., Caveolin-1 regulates transforming growth factor (TGF)-beta/SMAD signaling through an interaction with the TGF-beta type I receptor. J Biol Chem, 2001. 276(9): p. 6727-38.

103. Fujita, Y., et al., Caveolin-1 in mesangial cells suppresses MAP kinase activation and cell proliferation induced by bFGF and PDGF. Kidney Int, 2004. 66(5): p. 1794-804.

104. Lin, D., et al., Protein kinase Cgamma regulation of gap junction activity through caveolin-1-containing lipid rafts. Invest Ophthalmol Vis Sci, 2003. 44(12): p. 5259-68.

105. Toya, Y., et al., Inhibition of adenylyl cyclase by caveolin peptides. Endocrinology, 1998. 139(4): p. 2025-31.

106. Huang, C., et al., Organization of $G$ proteins and adenylyl cyclase at the plasma membrane. Mol Biol Cell, 1997. 8(12): p. 2365-78.

107. Schwencke, C., et al., Compartmentation of cyclic adenosine 3',5'-monophosphate signaling in caveolae. Mol Endocrinol, 1999. 13(7): p. 1061-70.

108. Ostrom, R.S., et al., Localization of adenylyl cyclase isoforms and G protein-coupled receptors in vascular smooth muscle cells: expression in caveolin-rich and noncaveolin domains. Mol Pharmacol, 2002. 62(5): p. 983-92.

109. Lu, M.L., et al., Caveolin-1 interacts with androgen receptor. A positive modulator of androgen receptor mediated transactivation. J Biol Chem, 2001. 276(16): p. 13442-51.

110. Chambliss, K.L., et al., Estrogen receptor alpha and endothelial nitric oxide synthase are organized into a functional signaling module in caveolae. Circ Res, 2000. 87(11): p. E4452.

111. Schlegel, A., et al., Caveolin-1 potentiates estrogen receptor alpha (ERalpha) signaling. caveolin-1 drives ligand-independent nuclear translocation and activation of ERalpha. $\mathrm{J}$ Biol Chem, 1999. 274(47): p. 33551-6.

112. Ghosh, S., et al., Interaction between caveolin-1 and the reductase domain of endothelial nitric-oxide synthase. Consequences for catalysis. J Biol Chem, 1998. 273(35): p. 2226771.

113. Ju, H., et al., Direct interaction of endothelial nitric-oxide synthase and caveolin-1 inhibits synthase activity. J Biol Chem, 1997. 272(30): p. 18522-5.

114. Venema, V.J., et al., Caveolin-1 detergent solubility and association with endothelial nitric oxide synthase is modulated by tyrosine phosphorylation. Biochem Biophys Res Commun, 1997. 236(1): p. 155-61.

115. Kiss, A.L., et al., Caveolin isoforms in resident and elicited rat peritoneal macrophages. Eur J Cell Biol, 2000. 79(5): p. 343-9.

116. Shin, J.S., Z. Gao, and S.N. Abraham, Involvement of cellular caveolae in bacterial entry into mast cells. Science, 2000. 289(5480): p. 785-8.

117. Werling, D., et al., Involvement of caveolae in the uptake of respiratory syncytial virus antigen by dendritic cells. J Leukoc Biol, 1999. 66(1): p. 50-8.

118. Yan, S.R., L. Fumagalli, and G. Berton, Activation of SRC family kinases in human neutrophils. Evidence that p58C-FGR and p53/56LYN redistributed to a Triton X-100- 
insoluble cytoskeletal fraction, also enriched in the caveolar protein caveolin, display an enhanced kinase activity. FEBS Lett, 1996. 380(1-2): p. 198-203.

119. Harris, J., et al., Expression of caveolin by bovine lymphocytes and antigen-presenting cells. Immunology, 2002. 105(2): p. 190-5.

120. Hu, G., et al., Neutrophil caveolin-1 expression contributes to mechanism of lung inflammation and injury. Am J Physiol Lung Cell Mol Physiol, 2008. 294(2): p. L178-86.

121. Garrean, S., et al., Caveolin-1 regulates NF-kappaB activation and lung inflammatory response to sepsis induced by lipopolysaccharide. J Immunol, 2006. 177(7): p. 4853-60.

122. Gargalovic, P. and L. Dory, Cellular apoptosis is associated with increased caveolin-1 expression in macrophages. J Lipid Res, 2003. 44(9): p. 1622-32.

123. Gargalovic, P. and L. Dory, Caveolins and macrophage lipid metabolism. J Lipid Res, 2003. 44(1): p. 11-21.

124. Li, J., et al., Impaired phagocytosis in caveolin-1 deficient macrophages. Cell Cycle, 2005. 4(11): p. 1599-607.

125. Wang, X.M., et al., Caveolin-1 confers antiinflammatory effects in murine macrophages via the MKK3/p38 MAPK pathway. Am J Respir Cell Mol Biol, 2006. 34(4): p. 434-42.

126. Mulvey, M.A. and S.J. Hultgren, Cell biology. Bacterial spelunkers. Science, 2000. 289(5480): p. 732-3.

127. Kiss, A.L. and H.J. Geuze, Caveolae can be alternative endocytotic structures in elicited macrophages. Eur J Cell Biol, 1997. 73(1): p. 19-27.

128. Kiss, A.L., et al., Caveolae and caveolin isoforms in rat peritoneal macrophages. Micron, 2002. 33(1): p. 75-93.

129. Ohnuma, K., et al., Caveolin-1 triggers T-cell activation via CD26 in association with CARMA1. J Biol Chem, 2007. 282(13): p. 10117-31.

130. Medina, F.A., et al., A novel role for caveolin-1 in B lymphocyte function and the development of thymus-independent immune responses. Cell Cycle, 2006. 5(16): p. 186571.

131. Chen, S.F., et al., Caveolin-1 facilitates cyclooxygenase-2 protein degradation. J Cell Biochem, 2010. 109(2): p. 356-62.

132. Tourkina, E., et al., Opposing effects of protein kinase Calpha and protein kinase Cepsilon on collagen expression by human lung fibroblasts are mediated via MEK/ERK and caveolin-1 signaling. J Biol Chem, 2005. 280(14): p. 13879-87.

133. Wang, X.M., et al., Caveolin-1: a critical regulator of lung fibrosis in idiopathic pulmonary fibrosis. J Exp Med, 2006. 203(13): p. 2895-906.

134. Wunderlich, C., et al., Chronic NOS inhibition prevents adverse lung remodeling and pulmonary arterial hypertension in caveolin-1 knockout mice. Pulm Pharmacol Ther, 2008. 21(3): p. 507-15.

135. Drab, M., et al., Loss of caveolae, vascular dysfunction, and pulmonary defects in caveolin-1 gene-disrupted mice. Science, 2001. 293(5539): p. 2449-52.

136. Mineo, C., et al., Localization of epidermal growth factor-stimulated Ras/Raf-1 interaction to caveolae membrane. J Biol Chem, 1996. 271(20): p. 11930-5.

137. Boyce, J.A., Eicosanoids in asthma, allergic inflammation, and host defense. Curr Mol Med, 2008. 8(5): p. 335-49.

138. Funk, C.D., Prostaglandins and leukotrienes: advances in eicosanoid biology. Science, 2001. 294(5548): p. 1871-5. 
139. Huang, S.K. and M. Peters-Golden, Eicosanoid lipid mediators in fibrotic lung diseases: ready for prime time? Chest, 2008. 133(6): p. 1442-50.

140. Charbeneau, R.P. and M. Peters-Golden, Eicosanoids: mediators and therapeutic targets in fibrotic lung disease. Clin Sci (Lond), 2005. 108(6): p. 479-91.

141. Six, D.A. and E.A. Dennis, The expanding superfamily of phospholipase A(2) enzymes: classification and characterization. Biochim Biophys Acta, 2000. 1488(1-2): p. 1-19.

142. Berenbaum, F., et al., Concomitant recruitment of ERK1/2 and p38 MAPK signalling pathway is required for activation of cytoplasmic phospholipase A2 via ATP in articular chondrocytes. J Biol Chem, 2003. 278(16): p. 13680-7.

143. Evans, J.H., et al., Intracellular calcium signals regulating cytosolic phospholipase A2 translocation to internal membranes. J Biol Chem, 2001. 276(32): p. 30150-60.

144. Smith, T., et al., Arachidonic acid and nonsteroidal anti-inflammatory drugs induce conformational changes in the human prostaglandin endoperoxide H2 synthase-2 (cyclooxygenase-2). J Biol Chem, 2000. 275(51): p. 40407-15.

145. Murakami, M., et al., Prostaglandin endoperoxide synthase-1 and -2 couple to different transmembrane stimuli to generate prostaglandin D2 in mouse bone marrow-derived mast cells. J Biol Chem, 1994. 269(35): p. 22269-75.

146. Rhee, S.H. and D. Hwang, Murine TOLL-like receptor 4 confers lipopolysaccharide responsiveness as determined by activation of $N F$ kappa $B$ and expression of the inducible cyclooxygenase. J Biol Chem, 2000. 275(44): p. 34035-40.

147. Jakobsson, P.J., et al., Identification of human prostaglandin E synthase: a microsomal, glutathione-dependent, inducible enzyme, constituting a potential novel drug target. Proc Natl Acad Sci U S A, 1999. 96(13): p. 7220-5.

148. Mancini, J.A., et al., Cloning, expression, and up-regulation of inducible rat prostaglandin e synthase during lipopolysaccharide-induced pyresis and adjuvantinduced arthritis. J Biol Chem, 2001. 276(6): p. 4469-75.

149. Hirai, H., et al., Prostaglandin D2 selectively induces chemotaxis in Thelper type 2 cells, eosinophils, and basophils via seven-transmembrane receptor CRTH2. J Exp Med, 2001. 193(2): p. 255-61.

150. Monneret, G., et al., Prostaglandin D2 is a potent chemoattractant for human eosinophils that acts via a novel DP receptor. Blood, 2001. 98(6): p. 1942-8.

151. Kobayashi, T. and S. Narumiya, Function of prostanoid receptors: studies on knockout mice. Prostaglandins Other Lipid Mediat, 2002. 68-69: p. 557-73.

152. Boie, Y., et al., Cloning and expression of a cDNA for the human prostanoid IP receptor. J Biol Chem, 1994. 269(16): p. 12173-8.

153. Kaliner, M. and K.F. Austen, Cyclic AMP, ATP, and reversed anaphylactic histamine release from rat mast cells. J Immunol, 1974. 112(2): p. 664-74.

154. Peachell, P.T., et al., Regulation of human basophil and lung mast cell function by cyclic adenosine monophosphate. J Immunol, 1988. 140(2): p. 571-9.

155. Stone, K.D., C. Prussin, and D.D. Metcalfe, IgE, mast cells, basophils, and eosinophils. J Allergy Clin Immunol, 2010. 125(2 Suppl 2): p. S73-80.

156. Prussin, C. and D.D. Metcalfe, 5. IgE, mast cells, basophils, and eosinophils. J Allergy Clin Immunol, 2006. 117(2 Suppl Mini-Primer): p. S450-6.

157. Prussin, C. and D.D. Metcalfe, 4. IgE, mast cells, basophils, and eosinophils. J Allergy Clin Immunol, 2003. 111(2 Suppl): p. S486-94. 
158. Abdel-Majid, R.M. and J.S. Marshall, Prostaglandin E2 induces degranulationindependent production of vascular endothelial growth factor by human mast cells. $\mathrm{J}$ Immunol, 2004. 172(2): p. 1227-36.

159. Goulet, J.L., et al., E-prostanoid-3 receptors mediate the proinflammatory actions of prostaglandin E2 in acute cutaneous inflammation. J Immunol, 2004. 173(2): p. 1321-6.

160. Feng, C., et al., Human mast cells express multiple EP receptors for prostaglandin E2 that differentially modulate activation responses. Blood, 2006. 107(8): p. 3243-50.

161. Bodor, J. and J.F. Habener, Role of transcriptional repressor ICER in cyclic AMPmediated attenuation of cytokine gene expression in human thymocytes. J Biol Chem, 1998. 273(16): p. 9544-51.

162. Luft, T., et al., Functionally distinct dendritic cell (DC) populations induced by physiologic stimuli: prostaglandin $E(2)$ regulates the migratory capacity of specific $D C$ subsets. Blood, 2002. 100(4): p. 1362-72.

163. Scandella, E., et al., Prostaglandin E2 is a key factor for CCR7 surface expression and migration of monocyte-derived dendritic cells. Blood, 2002. 100(4): p. 1354-61.

164. Jing, H., E. Vassiliou, and D. Ganea, Prostaglandin E2 inhibits production of the inflammatory chemokines CCL3 and CCL4 in dendritic cells. J Leukoc Biol, 2003. 74(5): p. 868-79.

165. Vassiliou, E., H. Jing, and D. Ganea, Prostaglandin E2 inhibits TNF production in murine bone marrow-derived dendritic cells. Cell Immunol, 2003. 223(2): p. 120-32.

166. Legler, D.F., et al., Prostaglandin E2 is generally required for human dendritic cell migration and exerts its effect via EP2 and EP4 receptors. J Immunol, 2006. 176(2): p. 966-73.

167. Kabashima, K., et al., Prostaglandin E2-EP4 signaling initiates skin immune responses by promoting migration and maturation of Langerhans cells. Nat Med, 2003. 9(6): p. 744-9.

168. Krause, P., et al., Prostaglandin E(2) enhances T-cell proliferation by inducing the costimulatory molecules OX4OL, CD70, and 4-1BBL on dendritic cells. Blood, 2009. 113(11): p. 2451-60.

169. Harris, S., Padilla, J., Koumas, L., Ray, D. \& Phipps, P., Prostaglandins as modulators of immunity. Trends in Immunology, 2002. 33(3): p. 144-150.

170. Ruggeri, P., et al., Polyamine metabolism in prostaglandin E2-treated human $T$ lymphocytes. Immunopharmacol Immunotoxicol, 2000. 22(1): p. 117-29.

171. Cosme, R., et al., Prostanoids in human colonic mucosa: effects of inflammation on $P G E(2)$ receptor expression. Hum Immunol, 2000. 61(7): p. 684-96.

172. Mastino, A., et al., Induction of apoptosis in thymocytes by prostaglandin E2 in vivo. Dev Immunol, 1992. 2(4): p. 263-71.

173. Pica, F., et al., Prostaglandin E2 induces apoptosis in resting immature and mature human lymphocytes: a c-Myc-dependent and Bcl-2-independent associated pathway. J Pharmacol Exp Ther, 1996. 277(3): p. 1793-800.

174. Porter, B.O. and T.R. Malek, Prostaglandin E2 inhibits T cell activation-induced apoptosis and Fas-mediated cellular cytotoxicity by blockade of Fas-ligand induction. Eur J Immunol, 1999. 29(7): p. 2360-5.

175. Sheibanie, A.F., et al., Prostaglandin E2 induces IL-23 production in bone marrowderived dendritic cells. FASEB J, 2004. 18(11): p. 1318-20. 
176. Yao, C., et al., Prostaglandin E2-EP4 signaling promotes immune inflammation through Th1 cell differentiation and Th17 cell expansion. Nat Med, 2009. 15(6): p. 633-40.

177. Roberts, L.J., 2nd, et al., Increased production of prostaglandin D2 in patients with systemic mastocytosis. N Engl J Med, 1980. 303(24): p. 1400-4.

178. Kawata, R., et al., Prostaglandin synthase 1 and prostaglandin synthase 2 both participate in activation-induced prostaglandin D2 production in mast cells. J Immunol, 1995. 155(2): p. 818-25.

179. Shichijo, M., et al., The effects of anti-asthma drugs on mediator release from cultured human mast cells. Clin Exp Allergy, 1998. 28(10): p. 1228-36.

180. Urade, Y., et al., The major source of endogenous prostaglandin D2 production is likely antigen-presenting cells. Localization of glutathione-requiring prostaglandin D synthetase in histiocytes, dendritic, and Kupffer cells in various rat tissues. J Immunol, 1989. 143(9): p. 2982-9.

181. Tanaka, K., et al., Cutting edge: differential production of prostaglandin D2 by human helper T cell subsets. J Immunol, 2000. 164(5): p. 2277-80.

182. Hardy, C.C., et al., The bronchoconstrictor effect of inhaled prostaglandin D2 in normal and asthmatic men. N Engl J Med, 1984. 311(4): p. 209-13.

183. Emery, D.L., et al., Prostaglandin D2 causes accumulation of eosinophils in the lumen of the dog trachea. J Appl Physiol, 1989. 67(3): p. 959-62.

184. Schuligoi, R., et al., Prostaglandin H2 induces the migration of human eosinophils through the chemoattractant receptor homologous molecule of Th2 cells, CRTH2. J Leukoc Biol, 2009. 85(1): p. 136-45.

185. Yoshimura-Uchiyama, C., et al., Differential modulation of human basophil functions through prostaglandin D2 receptors DP and chemoattractant receptor-homologous molecule expressed on Th2 cells/DP2. Clin Exp Allergy, 2004. 34(8): p. 1283-90.

186. Woodward, D.F., et al., Studies on the ocular pharmacology of prostaglandin D2. Invest Ophthalmol Vis Sci, 1990. 31(1): p. 138-46.

187. Woodward, D.F., et al., Further studies on ocular responses to DP receptor stimulation. Eur J Pharmacol, 1993. 230(3): p. 327-33.

188. Nagata, K., et al., Selective expression of a novel surface molecule by human Th2 cells in vivo. J Immunol, 1999. 162(3): p. 1278-86.

189. Sawyer, N., et al., Molecular pharmacology of the human prostaglandin D2 receptor, CRTH2. Br J Pharmacol, 2002. 137(8): p. 1163-72.

190. Stubbs, V.E., et al., Indomethacin causes prostaglandin D(2)-like and eotaxin-like selective responses in eosinophils and basophils. J Biol Chem, 2002. 277(29): p. 2601220.

191. Hata, A.N. and R.M. Breyer, Pharmacology and signaling of prostaglandin receptors: multiple roles in inflammation and immune modulation. Pharmacol Ther, 2004. 103(2): p. 147-66.

192. Tsuri, T., et al., Bicyclo[2.2.1] heptane and 6,6-dimethylbicyclo[3.1.1] heptane derivatives: orally active, potent, and selective prostaglandin D2 receptor antagonists. J Med Chem, 1997. 40(22): p. 3504-7.

193. Arimura, A., et al., Prevention of allergic inflammation by a novel prostaglandin receptor antagonist, S-5751. J Pharmacol Exp Ther, 2001. 298(2): p. 411-9.

194. Matsuoka, T., et al., Prostaglandin D2 as a mediator of allergic asthma. Science, 2000. 287(5460): p. 2013-7. 
195. Cosmi, L., et al., CRTH2 is the most reliable marker for the detection of circulating human type 2 Th and type 2 T cytotoxic cells in health and disease. Eur J Immunol, 2000. 30(10): p. 2972-9.

196. Nagata, K., et al., CRTH2, an orphan receptor of T-helper-2-cells, is expressed on basophils and eosinophils and responds to mast cell-derived factor(s). FEBS Lett, 1999. 459(2): p. 195-9.

197. Gosset, P., et al., Prostaglandin D2 affects the maturation of human monocyte-derived dendritic cells: consequence on the polarization of naive Th cells. J Immunol, 2003. 170(10): p. 4943-52.

198. Nagai, H., et al., The effect of a thromboxane A2 receptor antagonist BAY-u-3405 on experimental allergic reactions. Prostaglandins, 1995. 50(2): p. 75-87.

199. Narita, S., K. Asakura, and A. Kataura, Effects of thromboxane A2 receptor antagonist (Bay u 3405) on nasal symptoms after antigen challenge in sensitized guinea pigs. Int Arch Allergy Immunol, 1996. 109(2): p. 161-6.

200. Gervais, F.G., et al., Selective modulation of chemokinesis, degranulation, and apoptosis in eosinophils through the PGD2 receptors CRTH2 and DP. J Allergy Clin Immunol, 2001. 108(6): p. 982-8.

201. Hammad, H., et al., Prostaglandin D2 inhibits airway dendritic cell migration and function in steady state conditions by selective activation of the D prostanoid receptor 1 . J Immunol, 2003. 171(8): p. 3936-40.

202. Bilyk, N. and P.G. Holt, Cytokine modulation of the immunosuppressive phenotype of pulmonary alveolar macrophage populations. Immunology, 1995. 86(2): p. 231-7.

203. Robbiani, D.F., et al., The leukotriene C(4) transporter MRP1 regulates CCL19 (MIP3beta, ELC)-dependent mobilization of dendritic cells to lymph nodes. Cell, 2000. 103(5): p. 757-68.

204. Gilroy, D.W., et al., Inducible cyclooxygenase may have anti-inflammatory properties. Nat Med, 1999. 5(6): p. 698-701.

205. Brewster, C.E., et al., Myofibroblasts and subepithelial fibrosis in bronchial asthma. Am J Respir Cell Mol Biol, 1990. 3(5): p. 507-11.

206. Zhang, S., et al., Growth factors secreted by bronchial epithelial cells control myofibroblast proliferation: an in vitro co-culture model of airway remodeling in asthma. Lab Invest, 1999. 79(4): p. 395-405.

207. Adler, K.B., et al., Actin-containing cells in human pulmonary fibrosis. Am J Pathol, 1981. 102(3): p. 427-37.

208. Desmouliere, A. and G. Gabbiani, Myofibroblast differentiation during fibrosis. Exp Nephrol, 1995. 3(2): p. 134-9.

209. Zhang, H.Y., M. Gharaee-Kermani, and S.H. Phan, Regulation of lung fibroblast alphasmooth muscle actin expression, contractile phenotype, and apoptosis by IL-1beta. J Immunol, 1997. 158(3): p. 1392-9.

210. Zhang, K., K.C. Flanders, and S.H. Phan, Cellular localization of transforming growth factor-beta expression in bleomycin-induced pulmonary fibrosis. Am J Pathol, 1995. 147(2): p. 352-61.

211. Zhang, K., et al., Lung monocyte chemoattractant protein-1 gene expression in bleomycin-induced pulmonary fibrosis. J Immunol, 1994. 153(10): p. 4733-41.

212. Phan, S.H., Role of the myofibroblast in pulmonary fibrosis. Kidney Int Suppl, 1996. 54: p. S46-8. 
213. Bitterman, P.B., et al., Modulation of alveolar macrophage-driven fibroblast proliferation by alternative macrophage mediators. J Clin Invest, 1986. 77(3): p. 700-8.

214. Lama, V., et al., Prostaglandin E2 synthesis and suppression of fibroblast proliferation by alveolar epithelial cells is cyclooxygenase-2-dependent. Am J Respir Cell Mol Biol, 2002. 27(6): p. 752-8.

215. Huang, S.K., et al., Prostaglandin E(2) induces fibroblast apoptosis by modulating multiple survival pathways. FASEB J, 2009. 23(12): p. 4317-26.

216. Kolodsick, J.E., et al., Prostaglandin E2 inhibits fibroblast to myofibroblast transition via E. prostanoid receptor 2 signaling and cyclic adenosine monophosphate elevation. Am J Respir Cell Mol Biol, 2003. 29(5): p. 537-44.

217. White, K.E., et al., Prostaglandin E2 mediates IL-1beta-related fibroblast mitogenic effects in acute lung injury through differential utilization of prostanoid receptors. $\mathrm{J}$ Immunol, 2008. 180(1): p. 637-46.

218. White, E.S., et al., Prostaglandin E(2) inhibits fibroblast migration by E-prostanoid 2 receptor-mediated increase in PTEN activity. Am J Respir Cell Mol Biol, 2005. 32(2): p. 135-41.

219. Huang, S., et al., Prostaglandin E(2) inhibits collagen expression and proliferation in patient-derived normal lung fibroblasts via E prostanoid 2 receptor and cAMP signaling. Am J Physiol Lung Cell Mol Physiol, 2007. 292(2): p. L405-13.

220. Fine, A., et al., The differential effect of prostaglandin E2 on transforming growth factorbeta and insulin-induced collagen formation in lung fibroblasts. J Biol Chem, 1989. 264(29): p. 16988-91.

221. Moore, B.B., et al., GM-CSF regulates bleomycin-induced pulmonary fibrosis via a prostaglandin-dependent mechanism. J Immunol, 2000. 165(7): p. 4032-9.

222. Keerthisingam, C.B., et al., Cyclooxygenase-2 deficiency results in a loss of the antiproliferative response to transforming growth factor-beta in human fibrotic lung fibroblasts and promotes bleomycin-induced pulmonary fibrosis in mice. Am J Pathol, 2001. 158(4): p. 1411-22.

223. Desmouliere, A., [The healing of wounds. Biology and physiopathology]. Soins, 1995(595): p. 5-9.

224. Hansson, G.K., et al., Interferon gamma inhibits both proliferation and expression of differentiation-specific alpha-smooth muscle actin in arterial smooth muscle cells. J Exp Med, 1989. 170(5): p. 1595-608.

225. Desmouliere, A., et al., Alpha-smooth muscle actin is expressed in a subpopulation of cultured and cloned fibroblasts and is modulated by gamma-interferon. Exp Cell Res, 1992. 201(1): p. 64-73.

226. White, E.S., et al., Negative regulation of myofibroblast differentiation by PTEN (Phosphatase and Tensin Homolog Deleted on chromosome 10). Am J Respir Crit Care Med, 2006. 173(1): p. 112-21.

227. Huang, S.K., et al., Prostaglandin E2 inhibits specific lung fibroblast functions via selective actions of PKA and Epac-1. Am J Respir Cell Mol Biol, 2008. 39(4): p. 482-9.

228. Peters-Golden, M. and T.G. Brock, 5-lipoxygenase and FLAP. Prostaglandins Leukot Essent Fatty Acids, 2003. 69(2-3): p. 99-109.

229. Newcomer, M.E. and N.C. Gilbert, Location, Location, Location: Compartmentalization of Early Events in Leukotriene Biosynthesis. J Biol Chem, 2010. 
230. Steinhilber, D., 5-Lipoxygenase: enzyme expression and regulation of activity. Pharm Acta Helv, 1994. 69(1): p. 3-14.

231. Radmark, O. and B. Samuelsson, Regulation of the activity of 5-lipoxygenase, a key enzyme in leukotriene biosynthesis. Biochem Biophys Res Commun, 2010. 396(1): p. 105-10.

232. Murphy, R.C. and M.A. Gijon, Biosynthesis and metabolism of leukotrienes. Biochem J, 2007. 405(3): p. 379-95.

233. Peters-Golden, M. and W.R. Henderson, Jr., Leukotrienes. N Engl J Med, 2007. 357(18): p. 1841-54.

234. Folco, G. and R.C. Murphy, Eicosanoid transcellular biosynthesis: from cell-cell interactions to in vivo tissue responses. Pharmacol Rev, 2006. 58(3): p. 375-88.

235. Tager, A.M. and A.D. Luster, BLT1 and BLT2: the leukotriene B(4) receptors. Prostaglandins Leukot Essent Fatty Acids, 2003. 69(2-3): p. 123-34.

236. Kanaoka, Y. and J.A. Boyce, Cysteinyl leukotrienes and their receptors: cellular distribution and function in immune and inflammatory responses. J Immunol, 2004. 173(3): p. 1503-10.

237. Peters-Golden, M., Expanding roles for leukotrienes in airway inflammation. Curr Allergy Asthma Rep, 2008. 8(4): p. 367-73.

238. Palmblad, J., et al., Leukotriene B4 is a potent and stereospecific stimulator of neutrophil chemotaxis and adherence. Blood, 1981. 58(3): p. 658-61.

239. Shindo, K., M. Fukumura, and K. Miyakawa, Leukotriene B4 levels in the arterial blood of asthmatic patients and the effects of prednisolone. Eur Respir J, 1995. 8(4): p. 605-10.

240. Brach, M.A., et al., Leukotriene B4 transcriptionally activates interleukin-6 expression involving NK-chi B and NF-IL6. Eur J Immunol, 1992. 22(10): p. 2705-11.

241. Ford-Hutchinson, A.W., Leukotriene B4 and neutrophil function: a review. J R Soc Med, 1981. 74(11): p. 831-3.

242. Burke, J.R., et al., Leukotriene B4 stimulates the release of arachidonate in human neutrophils via the action of cytosolic phospholipase A2. Biochim Biophys Acta, 1997. 1359(1): p. 80-8.

243. Hafstrom, I., et al., Leukotriene B4--a stereospecific stimulator for release of lysosomal enzymes from neutrophils. FEBS Lett, 1981. 130(1): p. 146-8.

244. Weller, C.L., et al., Leukotriene B4, an activation product of mast cells, is a chemoattractant for their progenitors. J Exp Med, 2005. 201(12): p. 1961-71.

245. Miyahara, N., et al., Role of the LTB4/BLT1 pathway in allergen-induced airway hyperresponsiveness and inflammation. Allergol Int, 2006. 55(2): p. 91-7.

246. Miyahara, N., et al., Leukotriene B4 receptor 1 expression on dendritic cells is required for the development of Th2 responses and allergen-induced airway hyperresponsiveness. J Immunol, 2008. 181(2): p. 1170-8.

247. Terawaki, K., et al., Absence of leukotriene B4 receptor 1 confers resistance to airway hyperresponsiveness and Th2-type immune responses. J Immunol, 2005. 175(7): p. 421725.

248. Dahlen, S.E., et al., Leukotrienes are potent constrictors of human bronchi. Nature, 1980. 288(5790): p. 484-6.

249. Hanna, C.J., et al., Slow-reacting substances (leukotrienes) contract human airway and pulmonary vascular smooth muscle in vitro. Nature, 1981. 290(5804): p. 343-4. 
250. Bautz, F., et al., Chemotaxis and transendothelial migration of CD34(+) hematopoietic progenitor cells induced by the inflammatory mediator leukotriene D4 are mediated by the 7-transmembrane receptor CysLT1. Blood, 2001. 97(11): p. 3433-40.

251. Thivierge, M., J. Stankova, and M. Rola-Pleszczynski, $I L-13$ and $I L-4$ up-regulate cysteinyl leukotriene 1 receptor expression in human monocytes and macrophages. $\mathrm{J}$ Immunol, 2001. 167(5): p. 2855-60.

252. Resnick, M.B. and P.F. Weller, Mechanisms of eosinophil recruitment. Am J Respir Cell Mol Biol, 1993. 8(4): p. 349-55.

253. Braccioni, F., et al., The effect of cysteinyl leukotrienes on growth of eosinophil progenitors from peripheral blood and bone marrow of atopic subjects. J Allergy Clin Immunol, 2002. 110(1): p. 96-101.

254. Yamamoto, H., J.B. Sedgwick, and W.W. Busse, Differential regulation of eosinophil adhesion and transmigration by pulmonary microvascular endothelial cells. J Immunol, 1998. 161(2): p. 971-7.

255. Nagata, M., et al., Leukotriene D4 upregulates eosinophil adhesion via the cysteinyl leukotriene 1 receptor. J Allergy Clin Immunol, 2002. 109(4): p. 676-80.

256. Fregonese, L., et al., Cysteinyl leukotrienes induce human eosinophil locomotion and adhesion molecule expression via a CysLT1 receptor-mediated mechanism. Clin Exp Allergy, 2002. 32(5): p. 745-50.

257. Lee, E., et al., Leukotriene receptor antagonists and synthesis inhibitors reverse survival in eosinophils of asthmatic individuals. Am J Respir Crit Care Med, 2000. 161(6): p. 1881-6.

258. Machida, I., et al., Cysteinyl leukotrienes regulate dendritic cell functions in a murine model of asthma. J Immunol, 2004. 172(3): p. 1833-8.

259. Mosmann, T.R. and S. Sad, The expanding universe of T-cell subsets: Th1, Th2 and more. Immunol Today, 1996. 17(3): p. 138-46.

260. Kalinski, P., et al., T-cell priming by type-1 and type-2 polarized dendritic cells: the concept of a third signal. Immunol Today, 1999. 20(12): p. 561-7.

261. Langenkamp, A., et al., Kinetics of dendritic cell activation: impact on priming of TH1, TH2 and nonpolarized T cells. Nat Immunol, 2000. 1(4): p. 311-6.

262. Vieira, P.L., et al., Development of Th1-inducing capacity in myeloid dendritic cells requires environmental instruction. J Immunol, 2000. 164(9): p. 4507-12.

263. Muller, G., et al., Interleukin-10-treated dendritic cells modulate immune responses of naive and sensitized T cells in vivo. J Invest Dermatol, 2002. 119(4): p. 836-41.

264. Hamelmann, E., U. Wahn, and E.W. Gelfand, Role of the Th2 cytokines in the development of allergen-induced airway inflammation and hyperresponsiveness. Int Arch Allergy Immunol, 1999. 118(2-4): p. 90-4.

265. Mazzarella, G., et al., Th1/Th2 lymphocyte polarization in asthma. Allergy, 2000. 55 Suppl 61: p. 6-9.

266. Miyahara, N., et al., Requirement for leukotriene B4 receptor 1 in allergen-induced airway hyperresponsiveness. Am J Respir Crit Care Med, 2005. 172(2): p. 161-7.

267. Vargaftig, B.B. and M. Singer, Leukotrienes mediate murine bronchopulmonary hyperreactivity, inflammation, and part of mucosal metaplasia and tissue injury induced by recombinant murine interleukin-13. Am J Respir Cell Mol Biol, 2003. 28(4): p. 410-9.

268. Kim, D.C., et al., Cysteinyl leukotrienes regulate Th2 cell-dependent pulmonary inflammation. J Immunol, 2006. 176(7): p. 4440-8. 
269. Miyahara, N., et al., Leukotriene B4 receptor-1 is essential for allergen-mediated recruitment of CD8+T cells and airway hyperresponsiveness. J Immunol, 2005. 174(8): p. 4979-84.

270. Vannella, K.M., et al., Cysteinyl leukotrienes are autocrine and paracrine regulators of fibrocyte function. J Immunol, 2007. 179(11): p. 7883-90.

271. Beller, T.C., et al., Cysteinyl leukotriene 1 receptor controls the severity of chronic pulmonary inflammation and fibrosis. Proc Natl Acad Sci U S A, 2004. 101(9): p. 304752.

272. Hay, D.W., Pharmacology of leukotriene receptor antagonists. More than inhibitors of bronchoconstriction. Chest, 1997. 111(2 Suppl): p. 35S-45S.

273. Ozaki, T., et al., Regulatory effect of prostaglandin E2 on fibronectin release from human alveolar macrophages. Am Rev Respir Dis, 1990. 141(4 Pt 1): p. 965-9.

274. Wardlaw, A.J., et al., Leukotrienes, LTC4 and LTB4, in bronchoalveolar lavage in bronchial asthma and other respiratory diseases. J Allergy Clin Immunol, 1989. 84(1): p. 19-26.

275. Wilborn, J., et al., Constitutive activation of 5-lipoxygenase in the lungs of patients with idiopathic pulmonary fibrosis. J Clin Invest, 1996. 97(8): p. 1827-36.

276. Schroder, J.M., The monocyte-derived neutrophil activating peptide (NAP/interleukin 8) stimulates human neutrophil arachidonate-5-lipoxygenase, but not the release of cellular arachidonate. J Exp Med, 1989. 170(3): p. 847-63.

277. Hsieh, F.H., et al., Thelper cell type 2 cytokines coordinately regulate immunoglobulin E-dependent cysteinyl leukotriene production by human cord blood-derived mast cells: profound induction of leukotriene C(4) synthase expression by interleukin 4. J Exp Med, 2001. 193(1): p. 123-33.

278. Cowburn, A.S., S.T. Holgate, and A.P. Sampson, IL-5 increases expression of 5lipoxygenase-activating protein and translocates 5-lipoxygenase to the nucleus in human blood eosinophils. J Immunol, 1999. 163(1): p. 456-65.

279. Rankin, J.A., et al., IgE-dependent release of leukotriene C4 from alveolar macrophages. Nature, 1982. 297(5864): p. 329-31.

280. Matsukawa, A., et al., Endogenous monocyte chemoattractant protein-1 (MCP-1) protects mice in a model of acute septic peritonitis: cross-talk between $M C P-1$ and leukotriene B4. J Immunol, 1999. 163(11): p. 6148-54.

281. Egger, D., et al., IL-4 renders mast cells functionally responsive to endothelin-1. J Immunol, 1995. 154(4): p. 1830-7.

282. Riddick, C.A., et al., TGF-beta increases leukotriene C4 synthase expression in the monocyte-like cell line, THP-1. J Immunol, 1999. 162(2): p. 1101-7.

283. Steinhilber, D., O. Radmark, and B. Samuelsson, Transforming growth factor beta upregulates 5-lipoxygenase activity during myeloid cell maturation. Proc Natl Acad Sci U S A, 1993. 90(13): p. 5984-8.

284. Piedimonte, G., Neuroimmune interactions in respiratory syncytial virus-infected airways. Pediatr Infect Dis J, 2002. 21(5): p. 462-7.

285. Piedimonte, G., The association between respiratory syncytial virus infection and reactive airway disease. Respir Med, 2002. 96 Suppl B: p. S25-9.

286. Shay, D.K., et al., Bronchiolitis-associated hospitalizations among US children, 19801996. JAMA, 1999. 282(15): p. 1440-6. 
287. Falsey, A.R. and E.E. Walsh, Respiratory syncytial virus infection in adults. Clin Microbiol Rev, 2000. 13(3): p. 371-84.

288. Kim, H.W., et al., Epidemiology of respiratory syncytial virus infection in Washington, D.C. I. Importance of the virus in different respiratory tract disease syndromes and temporal distribution of infection. Am J Epidemiol, 1973. 98(3): p. 216-25.

289. Williams, J.V., et al., Human metapneumovirus and lower respiratory tract disease in otherwise healthy infants and children. N Engl J Med, 2004. 350(5): p. 443-50.

290. Miller, E.K., et al., Rhinovirus-associated hospitalizations in young children. J Infect Dis, 2007. 195(6): p. 773-81.

291. Kesebir, D., et al., Human bocavirus infection in young children in the United States: molecular epidemiological profile and clinical characteristics of a newly emerging respiratory virus. J Infect Dis, 2006. 194(9): p. 1276-82.

292. Piedimonte, G., Contribution of neuroimmune mechanisms to airway inflammation and remodeling during and after respiratory syncytial virus infection. Pediatr Infect Dis J, 2003. 22(2 Suppl): p. S66-74; discussion S74-5.

293. Kernie, S.G. and L.F. Parada, The molecular basis for understanding neurotrophins and their relevance to neurologic disease. Arch Neurol, 2000. 57(5): p. 654-7.

294. Lindsay, R.M. and A.J. Harmar, Nerve growth factor regulates expression of neuropeptide genes in adult sensory neurons. Nature, 1989. 337(6205): p. 362-4.

295. King, K.A., et al., Exaggerated neurogenic inflammation and substance P receptor upregulation in RSV-infected weanling rats. Am J Respir Cell Mol Biol, 2001. 24(2): p. 101-7.

296. Piedimonte, G., et al., Sendai virus infection potentiates neurogenic inflammation in the rat trachea. J Appl Physiol, 1990. 68(2): p. 754-60.

297. Smith, R.E., H.J. Zweerink, and W.K. Joklik, Polypeptide components of virions, top component and cores of reovirus type 3. Virology, 1969. 39(4): p. 791-810.

298. Saria, A., et al., Vascular protein linkage in various tissue induced by substance P, capsaicin, bradykinin, serotonin, histamine and by antigen challenge. Naunyn Schmiedebergs Arch Pharmacol, 1983. 324(3): p. 212-8.

299. Piedimonte, G., Pickles, RJ, Lehmann, JR, McCarty, D, Coasta, DL, Boucher, RC, Replication-deficient adenoviral vector for gene transfer potentiates airway neurogenic inflammation. Am J Respir Cell Mol Biol, 1997. 16(3): p. 250-258.

300. Piedimonte, G., Pathophysiological mechanisms for the respiratory syncytial virusreactive airway disease link. Respir Res, 2002. 3 Suppl 1: p. S21-5.

301. Piedimonte, G., et al., Respiratory syncytial virus upregulates expression of the substance $P$ receptor in rat lungs. Am J Physiol, 1999. 277(4 Pt 1): p. L831-40.

302. Rouissi, N., et al., Selectivity and specificity of new, non-peptide, quinuclidine antagonists of substance P. Biochem Biophys Res Commun, 1991. 176(2): p. 894-901.

303. Di Maria, G.U., S. Bellofiore, and P. Geppetti, Regulation of airway neurogenic inflammation by neutral endopeptidase. Eur Respir J, 1998. 12(6): p. 1454-62.

304. Weinstock, J.V., et al., Eosinophils from granulomas in murine schistosomiasis mansoni produce substance P. J Immunol, 1988. 141(3): p. 961-6.

305. Pascual, D.W., et al., The cytokine-like action of substance P upon B cell differentiation. Reg Immunol, 1992. 4(2): p. 100-4.

306. Bost, K.L. and D.W. Pascual, Substance P: a late-acting B lymphocyte differentiation cofactor. Am J Physiol, 1992. 262(3 Pt 1): p. C537-45. 
307. Killingsworth, C.R., et al., Rat alveolar macrophages express preprotachykinin gene-I mRNA-encoding tachykinins. Am J Physiol, 1997. 273(5 Pt 1): p. L1073-81.

308. Joos, G.F., P.R. Germonpre, and R.A. Pauwels, Role of tachykinins in asthma. Allergy, 2000. 55(4): p. 321-37.

309. Stanisz, A.M., D. Befus, and J. Bienenstock, Differential effects of vasoactive intestinal peptide, substance $P$, and somatostatin on immunoglobulin synthesis and proliferations by lymphocytes from Peyer's patches, mesenteric lymph nodes, and spleen. J Immunol, 1986. 136(1): p. 152-6.

310. Lotz, M., J.H. Vaughan, and D.A. Carson, Effect of neuropeptides on production of inflammatory cytokines by human monocytes. Science, 1988. 241(4870): p. 1218-21.

311. Bost, K.L., S.A. Breeding, and D.W. Pascual, Modulation of the mRNAs encoding substance P and its receptor in rat macrophages by LPS. Reg Immunol, 1992. 4(2): p. 105-12. 


\section{CURRICULUM VITAE}

\section{Maret Elizabeth Bernard}

1320 Bennington Avenue, Pittsburgh, PA 15217

Phone (304) 282-5089

Email: maretb@gmail.com

\section{EDUCATION}

M.S. in Biomedical Sciences

Department of Microbiology and Immunology

West Virginia University School of Medicine, WV

Advisor: Dr. Mario Scuri
December 2010

GPA 3.43/4.0

B.A. in Anthropology, concentration in Premedicine College of the Holy Cross, Worcester, Massachusettes

May 2005

GPA

\section{POSITIONS HELD}

Graduate Researcher

2007-2010

Department of Pediatrics

West Virginia University School of Medicine, Morgantown WV

Research Advisor: Dr. Mario Scuri

Research focused on discovering links between childhood exposure to infection and/or pollutants and lung diseases. Utilizing a rat model of viral respiratory tract infection to study changes in neurogenic inflammation in relation to asthma pathogenesis and airway remodeling.

Techniques: Cell culture, Western Blot, ELISA, RT-PCR, Real Time PCR, Histology, Animal care and surgery.

\section{Graduate Researcher}

Department of Microbiology and Immunology,

West Virginia University School of Medicine, Morgantown, WV

Research Advisor: Dr. Christopher Cuff

Investigated the cellular and molecular events that result in the development of antigen-specific immune responses in the gastrointestinal tract by utilizing a murine model of enteric infection as well as human intestinal tissues. The mucosal immune response to stimulation with virus was characterized using flow cytometry.

Techniques: Cell culture, Preparation of Reovirus stock, Antibody Staining and Flow Cytometry

\section{Research Fellow}

Summer 2003-2005

The Western Pennsylvania Prostate Foundation, Pittsburgh, PA

Research Advisor: Dr. John Maier 
Evaluated the unique biochemical composition of different materials or samples by utilizing Raman molecule imaging (RMI) to generate spectra. Information from spectra was used to distinguish chemical differences between different samples. The primary objective was to determine if RMI could provide clinically useful information in prostate cancer diagnosis and help to differentiate metastatic disease.

Techniques: Operation of FALCON II Raman Imaging Microscope (Chemimage), sample preparation \& spectra analysis.

\section{TEACHING EXPERIENCE}

\section{Teaching Assistant}

West Virginia University School of Dentistry

Dental Microbiology Fall 2006 and Fall 2008

\section{PRESENTATIONS}

May 2009

American Thoracic Society 2009 International Conference, San Diego, CA May 2009

"The Effects of Respiratory Enteric Orphan Virus (Reovirus) Infection on Airway

Remodeling in Rats"

May 2008

American Thoracic Society 2008 International Conference, Toronto, Canada "Respiratory Enteric Orphan Virus (Reovirus) Potentiates Neurogenic Inflammation in Rat Airways"

May 2008

American Thoracic Society 2008 International Conference, Toronto, Canada

"Reovirus Infection Causes Lung Fibrosis in Weanling Rats"

April 2008

Van Liere Convocation and Research Day, Morgantown, WV

"Respiratory Enteric Orphan Virus Causes Fibrosis in Weanling Rats"

April 2007

Van Liere Convocation and Research Day, Morgantown, WV

"Respiratory Enteric Orphan Virus (Reovirus) and Neurogenic Inflammation in a Rat Model" 